ESAIM: COCV 28 (2022) 21

https://doi.org/10.1051/cocv/2022015
ESAIM: Control, Optimisation and Calculus of Variations

www.esaim-cocv.org

\title{
EQUILIBRIUM PRICE FORMATION WITH A MAJOR PLAYER AND ITS MEAN FIELD LIMIT*
}

\author{
MasaAki FujiI**(1) AND AKIHIKo TAKAhashi
}

\begin{abstract}
In this article, we consider the problem of equilibrium price formation in an incomplete securities market consisting of one major financial firm and a large number of minor firms. They carry out continuous trading via the securities exchange to minimize their cost while facing idiosyncratic and common noises as well as stochastic order flows from their individual clients. The equilibrium price process that balances demand and supply of the securities, including the functional form of the price impact for the major firm, is derived endogenously both in the market of finite population size and in the corresponding mean field limit.
\end{abstract}

Mathematics Subject Classification. 49N80, 91A16, 91B50, 91B70.

Received April 7, 2021. Accepted February 12, 2022.

\section{INTRODUCTION}

In the traditional setups for financial derivatives and portfolio theories, a security price process is given exogenously as a part of the model inputs. On the other hand, in the field of financial economics, the problem of equilibrium price formation has been one of the central issues, which seeks an appropriate price process that balances demand and supply of securities among a large number of agents endogenously based on their preferences and rational actions. The intrinsic difficulty for the latter comes from the strategic interactions among the agents.

The progress in the mean field game (MFG) theory in the last decade has opened a new promising approach to study the long-standing problem of multi-agent games. Since the publication of seminal works by Lasry and Lions [41-43] and Huang et al. [34-37], which characterizes the Nash equilibrium by a coupled system of Hamilton-Jacobi-Bellman (HJB) and Kolmogorov equations, the mean field game has been one of the central themes among many researchers.

Carmona and Delarue $[8,9]$ developed a probabilistic approach to the mean field games and mean-field type control problems based on a forward-backward stochastic differential equation (FBSDE) of McKean-Vlasov type. Lacker $[39,40]$ initiated the weak formulation of the mean field games by applying the relaxed-control technique. An extension to the so-called extended mean field games was recently made by Djete $[17,18]$. The mean field

* All the contents expressed in this research are solely those of the author and do not represent any views or opinions of any institutions. The author is not responsible or liable in any manner for any losses and/or damages caused by the use of any contents in this research.

Keywords and phrases: Mean field game, major agent, mean-field type control, controlled-FBSDEs, equilibrium price formation, market clearing.

Quantitative Finance Course, Graduate School of Economics, The University of Tokyo, Tokyo, Japan.

** Corresponding author: mfujii@e.u-tokyo.ac.jp

(C) The authors. Published by EDP Sciences, SMAI 2022

This is an Open Access article distributed under the terms of the Creative Commons Attribution License (https://creativecommons.org/licenses/by/4.0), which permits unrestricted use, distribution, and reproduction in any medium, provided the original work is properly cited. 
games in the presence of common noise were developed by Carmona et al. [12] in the framework of weak solutions. Laurière and Tangpi [46] generalized the concept of propagation of chaos for forward and backward weakly interacting particles. Since the mean field game theory can decompose a complicated Nash system arising from stochastic differential games into a separate optimization and an additional fixed point problem, it has found vast applications involving many homogeneous agents competing through symmetric interactions. For interested readers, there exist excellent monographs such as $[3,29,30,38]$ for analytic approach and $[10,11]$ for probabilistic approach. See also the lecture notes by Cardaliaguet [6].

Since the original MFG setting assumes the homogeneous agents, one natural extension is to allow multiple types of populations, where the cost functions as well as the coefficient functions of the state dynamics can be different population by population. See, for example, [2, 5, 15, 20, 54] for analytic approach and [26] for probabilistic approach. Another important direction of research is to allow the existence of a major agent whose importance does not diminish even in the large population limit of the minor agents. Huang [33] introduced linear-quadratic mean field games with a major agent, which was extended by Nourian and Caines [47] to a general nonlinear dynamical system. Bensoussan et al. [4] and Carmona and Zhu [13] further developed the framework to allow the major agent to directly influence the law of the minor agents. The former considered the Stackelberg equilibrium and the latter dealt with the Nash equilibrium. See also [23] for recent generalization in the linear-quadratic system, and $[7,44]$ which studies the master equation for the mean field games with a major agent.

These developments of the MFG theory have been successfully applied to various problems regarding in particular, the energy and financial markets which naturally involve a large number of agents with similar preferences. A popular phenomenological approach used to fit to the concept of Nash equilibrium is to assume that the relevant asset price is decomposed into two parts; one is a so-called fundamental price, which is exogenously given and assumed to be independent of the agents' actions, and the other part representing the market friction which is often assumed to be proportional to the average trading speed among the agents. One can find in $[1,14,16,21,24,32,45]$ interesting applications to, optimal trading, liquidation, energy production, optimal use of smart grids, etc. In particular, we refer to Fu and Horst [25], Evangelista and Thamsten [19] and Féron et al. [22] who studied the optimal liquidation and trading problems in the mean-field games with a major player.

As for the problem of equilibrium price formation, which requires the prices to balance demand and supply of the corresponding assets, application of the MFG theory has been surprisingly rare. The first contribution in this direction was made by Gomes and Saude [31] who modeled the electricity price process using the analytic approach. Recently, Shrivats et al. [48] and Fujii and Takahashi [27] independently proposed a probabilistic model for equilibrium price formation. In the former work, the authors studied the solar renewable energy certificate (SREC) market and derived the equilibrium SREC price using McKean-Vlasov FBSDEs. As in [31], they assumed that each agent is subject to an independent noise and applied the fixed-point technique developed by [8] to obtain a deterministic process for the equilibrium price. In the latter, we studied the price process of general financial assets using a stylized model of the securities exchange. In contrast to [31, 48], we included a common noise which affects all the agents. Since the existence of the common noise makes it impossible to use the fixed-point technique, we resorted to the continuation method developed by Yong [51] and Peng and Wu [49] to solve the conditional McKean-Vlasov FBSDEs directly under the appropriate monotone conditions. In the accompanying work [28], we proved the strong convergence of the finite agent equilibrium to the corresponding mean field limit given in [27]. Note that, if we only want a short-term solution, the monotone conditions are unnecessary. In economic terms, they prevent the price bubbles/crashes from happening so that the price process is well-posed for an arbitrary interval. Roughly speaking, they require the demand of the securities decreases when their prices rise.

In the current paper, we further developed the model studied in the two preceding works $[27,28]$ by including a major agent. As long as we know, this is the first attempt to solve the problem of equilibrium price formation with a major agent under the market-clearing condition. For a given order flow from the major agent, a properly functioning market is expected to produce an equilibrium price process as in [27, 28]. Since the equilibrium price process of the securities becomes dependent on the trading strategy of the major agent, her optimization problem 
ends up in minimizing the cost with her own feedback effects into account, which is given by a large system of controlled-FBSDEs in the case of the finite population market, and by controlled-FBSDEs of conditional McKean-Vlasov type in the limit of large population size. In order to guarantee the optimality, we prove the new verification theorem for the controlled-FBSDEs of conditional McKean-Vlasov type. Although we are forced to assume a linear-quadratic setup (with stochastic coefficients) for the minor agents in order to make the verification theorem hold, we keep a general non-linear cost function for the major agent. The resultant system of fully-coupled FBSDEs is solved once again by the continuation method. The equilibrium price process that balances demand and supply of the securities, including the functional form of the price impact for the major agent, is derived endogenously both in the market of finite population size and in the corresponding mean field limit. Lastly, we show the strong convergence of the finite agent equilibrium to the corresponding mean-field limit. Note that, it is quite rare that one can prove the strong convergence of $N$-agent equilibrium to the corresponding mean-field limit outside the explicitly solvable linear-quadratic (LQ) settings. To the best of the authors' knowledge, this is the first example of this kind in the presence of a major player in the non-LQ setups. As an important byproduct, we obtain the direct estimate on the difference of the equilibrium price between the two markets.

The organization of the paper is as follows. After explaining the notations in Section 2, we solve the equilibrium price formation for the finite population market in Section 3. The corresponding problem in the mean-field limit is solved in Section 4. In Section 5, we prove the strong convergence of the finite population equilibrium to the corresponding mean field limit and give the stability result for the market-clearing price between the two cases. Section 6 gives a brief discussion on the special case in which the securities have a specified date of maturity with exogenously determined payoffs, as in the case for Futures, Bonds and other financial derivatives. A general verification theorem for the optimization problem with respect to the controlled-FBSDEs is provided in Appendix.

\section{Notations}

We use the same notation adopted in the work [28]. We introduce $(N+1)$ complete probability spaces:

$$
\left(\bar{\Omega}^{0}, \overline{\mathcal{F}}^{0}, \overline{\mathbb{P}}^{0}\right) \text { and }\left(\bar{\Omega}^{i}, \overline{\mathcal{F}}^{i}, \overline{\mathbb{P}}^{i}\right)_{i=1}^{N},
$$

endowed with filtrations $\overline{\mathbb{F}}^{i}:=\left(\overline{\mathcal{F}}_{t}^{i}\right)_{t \geq 0}, i \in\{0, \ldots, N\}$. Here, $\overline{\mathbb{F}}^{0}$ is the completion of the filtration generated by $d^{0}$-dimensional Brownian motion $\boldsymbol{W}^{0}$ (hence right-continuous) and, for each $i \in\{1, \ldots, N\}, \overline{\mathbb{F}}^{i}$ is the complete and right-continuous augmentation of the filtration generated by $d$-dimensional Brownian motions $\boldsymbol{W}^{i}$ as well as a $\boldsymbol{W}^{i}$-independent $n$-dimensional square-integrable random variables $\left(\xi^{i}\right)$. We also introduce the product probability spaces

$$
\Omega^{i}=\bar{\Omega}^{0} \times \bar{\Omega}^{i}, \quad \mathcal{F}^{i}, \quad \mathbb{F}^{i}=\left(\mathcal{F}_{t}^{i}\right)_{t \geq 0}, \quad \mathbb{P}^{i}, i \in\{1, \ldots, N\}
$$

where $\left(\mathcal{F}^{i}, \mathbb{P}^{i}\right)$ is the completion of $\left(\overline{\mathcal{F}}^{0} \otimes \overline{\mathcal{F}}^{i}, \overline{\mathbb{P}}^{0} \otimes \overline{\mathbb{P}}^{i}\right)$ and $\mathbb{F}^{i}$ is the complete and right-continuous augmentation of $\left(\overline{\mathcal{F}}_{t}^{0} \otimes \overline{\mathcal{F}}_{t}^{i}\right)_{t \geq 0}$. In the same way, we define the complete probability space $(\Omega, \mathcal{F}, \mathbb{P})$ endowed with $\mathbb{F}=\left(\mathcal{F}_{t}\right)_{t \geq 0}$ satisfying the usual conditions as a product of $\left(\bar{\Omega}^{i}, \overline{\mathcal{F}}^{i}, \overline{\mathbb{P}}^{i} ; \overline{\mathbb{F}}^{i}\right)_{i=0}^{N}$.

Throughout the work, the symbol $L$ and $L_{0}$ denote given positive constants, the symbol $C$ a general positive constant which may change line by line. For a given constant $T>0$, we use the following notation for frequently encountered spaces:

$-\mathcal{S}_{+}^{n}$ denotes the space of $n \times n$ strictly positive definite matrices.

$-\mathcal{S}^{n}$ denotes the space of $n \times n$ positive semidefinite matrices.

- $\mathbb{L}^{2}\left(\mathcal{G} ; \mathbb{R}^{d}\right)$ denotes the set of $\mathbb{R}^{d}$-valued $\mathcal{G}$-measurable square integrable random variables. 
- $\mathbb{S}^{2}\left(\mathbb{G} ; \mathbb{R}^{d}\right)$ is the set of $\mathbb{R}^{d}$-valued $\mathbb{G}$-adapted continuous processes $\boldsymbol{X}$ satisfying

$$
\|X\|_{\mathbb{S}^{2}}:=\mathbb{E}\left[\sup _{t \in[0, T]}\left|X_{t}\right|^{2}\right]^{\frac{1}{2}}<\infty .
$$

- $\mathbb{H}^{2}\left(\mathbb{G} ; \mathbb{R}^{d}\right)$ is the set of $\mathbb{R}^{d}$-valued $\mathbb{G}$-progressively measurable processes $\boldsymbol{Z}$ satisfying

$$
\|Z\|_{\mathbb{H}^{2}}:=\mathbb{E}\left[\left(\int_{0}^{T}\left|Z_{t}\right|^{2} \mathrm{~d} t\right)\right]^{\frac{1}{2}}<\infty
$$

- $\mathcal{L}(X)$ denotes the law of a random variable $X$.

- $\mathcal{P}\left(\mathbb{R}^{d}\right)$ is the set of probability measures on $\left(\mathbb{R}^{d}, \mathcal{B}\left(\mathbb{R}^{d}\right)\right)$.

- $\mathcal{P}_{p}\left(\mathbb{R}^{d}\right)$ with $p \geq 1$ is the subset of $\mathcal{P}\left(\mathbb{R}^{d}\right)$ with finite $p$-th moment; i.e., the set of $\mu \in \mathcal{P}\left(\mathbb{R}^{d}\right)$ satisfying

$$
M_{p}(\mu):=\left(\int_{\mathbb{R}^{d}}|x|^{p} \mu(\mathrm{d} x)\right)^{\frac{1}{p}}<\infty
$$

We always assign $\mathcal{P}_{p}\left(\mathbb{R}^{d}\right)$ with $(p \geq 1)$ the $p$-Wasserstein distance $W_{p}$, which makes $\mathcal{P}_{p}\left(\mathbb{R}^{d}\right)$ a complete separable metric space. It is defined by, for any $\mu, \nu \in \mathcal{P}_{p}\left(\mathbb{R}^{d}\right)$,

$$
W_{p}(\mu, \nu):=\inf _{\pi \in \Pi_{p}(\mu, \nu)}\left[\left(\int_{\mathbb{R}^{d} \times \mathbb{R}^{d}}|x-y|^{p} \pi(\mathrm{d} x, \mathrm{~d} y)\right)^{\frac{1}{p}}\right]
$$

where $\Pi_{p}(\mu, \nu)$ denotes the set of probability measures in $\mathcal{P}_{p}\left(\mathbb{R}^{d} \times \mathbb{R}^{d}\right)$ with marginals $\mu$ and $\nu$. For more details, see Chapter 5 in [10].

- For any $N$ variables $\left(x^{i}\right)_{i=1}^{N}$, we write its empirical mean as

$$
\mathfrak{m}((x)):=\mathfrak{m}\left(\left(x^{i}\right)_{i=1}^{N}\right):=\frac{1}{N} \sum_{i=1}^{N} x^{i} .
$$

We frequently omit the arguments such as $\left(\mathbb{G}, \mathbb{R}^{d}\right)$ in the above definitions when there is no confusion from the context.

\section{Equilibrium of Finite population size}

\subsection{Problem description}

In the preceding works, we have been interested in the equilibrium price formation in a financial market among a large number of security firms. Every firm (agent) is supposed to have many individual clients who cannot directly access to the exchange. Therefore, every agent supposed to face the stochastic order flows from his individual clients in addition to the idiosyncratic as well as common market shocks. Under such an environment, they carry out optimal trading via the common exchange to minimize their cost functions. Importantly, since there exist very large number of agents, every agent considers that his market share is negligibly small and hence that there is no direct market impact from his trading. In other words, they behave as price takers. The problem of equilibrium price formation is to search an appropriate price process of securities which equalize the demand and supply based on the agents' cost functions and the state dynamics. In the presence of common shocks, the price process inevitably becomes stochastic. Such a problem has been investigated in our two preceding papers [27, 28], where the former treats the mean-field limit and the latter proves the strong convergence to the mean-field limit from the corresponding equilibrium of finite population. 
The new twist in the current paper is the presence of one major agent, a huge financial firm, who knows that her trading volume has a significant market share. For a given order flow from the major agent, a properly functioning market is expected to produce an equilibrium price process so that it matches the net demand and supply among all the agents. Through this function of the market, the equilibrium price process of the securities becomes dependent on the trading strategy of the major agent. Therefore, her optimization problem ends up in minimizing the cost with her own feedback effects into account. We then finally obtain the market equilibrium price process by solving the major agent's optimal strategy. In the following, we first solve this problem in the market with finite population size. The minor agents are allowed to be heterogeneous so that the coefficients functions for their state processes as well as the cost functions can be different from each other. The large population limit of minor agents will be studied in later sections.

Let us now describe the setup more concretely. There are $N$ minor agents indexed by $i=1, \ldots, N$. The major agent is always labeled by the index 0 . The number of securities traded in the market is assumed to be $n \in \mathbb{N}$. Each minor agent $i \in\{1, \ldots, N\}$ tries to solve the cost minimization problem among the admissible strategies $\mathbb{A}^{i}:=\mathbb{H}^{2}\left(\mathbb{F} ; \mathbb{R}^{n}\right)$

$$
\inf _{\boldsymbol{\alpha}^{i} \in \mathbb{A}^{i}} J^{i}\left(\boldsymbol{\alpha}^{i}\right)
$$

with some functions $f_{i}$ and $g_{i}$, which denotes the running as well as terminal costs, respectively:

$$
J^{i}\left(\boldsymbol{\alpha}^{i}\right):=\mathbb{E}\left[\int_{0}^{T} f_{i}\left(t, X_{t}^{i}, \alpha_{t}^{i}, \varpi_{t}, \Lambda_{t}, c_{t}^{0}, c_{t}^{i}\right) \mathrm{d} t+g_{i}\left(X_{T}^{i}, \varpi_{T}, c_{T}^{0}, c_{T}^{i}\right)\right]
$$

The dynamic constraint, which is the time evolution of the securities' position size of the $i$ th agent, is given by

$$
\mathrm{d} X_{t}^{i}=\left(\alpha_{t}^{i}+l_{i}\left(t, c_{t}^{0}, c_{t}^{i}\right)\right) \mathrm{d} t+\sigma_{i}^{0}\left(t, c_{t}^{0}, c_{t}^{i}\right) \mathrm{d} W_{t}^{0}+\sigma_{i}\left(t, c_{t}^{0}, c_{t}^{i}\right) \mathrm{d} W_{t}^{i}, \quad t \in[0, T]
$$

with $X_{0}^{i}=\xi^{i}$. Here, $\xi^{i} \in \mathbb{L}^{2}\left(\overline{\mathcal{F}}_{0}^{i} ; \mathbb{R}^{n}\right)$ denotes the size of the initial position, which is assumed to have the common law for every $1 \leq i \leq N$. $\left(\varpi_{t}\right)_{t \in[0, T]} \in \mathbb{H}^{2}\left(\mathbb{F} ; \mathbb{R}^{n}\right)$ denotes the market price process of the $n$ securities. In the end, we want to determine $\left(\varpi_{t}\right)_{t \in[0, T]}$ endogenously so that it equalizes the amount of demand and supply. $\left(c_{t}^{0}\right)_{t \geq 0} \in \mathbb{H}^{2}\left(\overline{\mathbb{F}}^{0} ; \mathbb{R}^{n}\right)$ with $c_{T}^{0} \in \mathbb{L}^{2}\left(\overline{\mathcal{F}}_{T}^{0} ; \mathbb{R}^{n}\right)$ denotes the coupon payments from the securities or the market news affecting all the agents, while $\left(c_{t}^{i}\right)_{t \geq 0} \in \mathbb{H}^{2}\left(\overline{\mathbb{F}}^{i} ; \mathbb{R}^{n}\right)$ with $c_{T}^{i} \in \mathbb{L}^{2}\left(\overline{\mathcal{F}}_{T}^{i} ; \mathbb{R}^{n}\right)$ denotes some idiosyncratic shocks affecting only the $i$ th agent. Moreover, $\left(c_{t}^{i}\right)_{t \geq 0}$ are also assumed to have the common law for all $1 \leq i \leq N$. $\left(\Lambda_{t}\right)_{t \in[0, T]}$ is an $\overline{\mathbb{F}}^{0}$-adapted process related to the trading fee to be paid to the exchange. The terms involving $\left(l_{i}, \sigma_{i}^{0}, \sigma_{i}\right)$ denote the order flow to the $i$ th agent from his individual clients through the over-the-counter (OTC) market. Each minor agent controls $\left(\alpha_{t}^{i}\right)_{t \in[0, T]}$, which is an $\mathbb{R}^{n}$-valued process denoting the trading speed of the $n$ securities via the exchange. More precisely, $\left(\alpha_{t}^{i}\right)^{k} \mathrm{~d} t, 1 \leq k \leq n$, denotes the number of shares of the $k$ th security bought (or sold if negative) within the time interval $[t, t+\mathrm{d} t]$ by the $i$ th agent. Note that, in addition to the random initial states $\left(\xi^{i}\right)_{i=1}^{N}$, we have $d_{0}$-dimensional common noise $W^{0}$ and $N d$-dimensional idiosyncratic noises $\left(W^{i}\right)_{i=1}^{N}$. Since we impose no restriction on the size among $\left(n, d_{0}, d, N\right)$, we have an incomplete securities market in general. For more information, see Section 3 of [27], which explains the financial interpretation of each term in details.

When the number of agents $N$ is sufficiently large, it is natural to assume that each minor agent consider himself as a price taker. Throughout the paper, we assume that this is the case. This means that each minor agent tries to solve the optimization problem by treating $\left(\varpi_{t}\right)_{t \geq 0}$ as an exogenous process. Suppose that the trading strategy of the major agent is given by $\left(\beta_{t}\right)_{t \in[0, T]}$, which denotes her trading speed. For given order flow $\left(\beta_{t}\right)_{t \in[0, T]}$, the financial market is expected to produce an equilibrium price process $\left(\varpi_{t}\right)_{t \in[0, T]}$ which equalizes the demand and supply among all the agents. Our first goal is to find such a price process $\left(\varpi_{t}\right)_{t \in[0, T]}$ which 
achieves

$$
\sum_{i=1}^{N} \widehat{\alpha}_{t}^{i}+\beta_{t}=0
$$

$\mathrm{d} t \otimes d \mathbb{P}$-a.e., where $\left(\left(\widehat{\alpha}_{t}^{i}\right)_{t \in[0, T]}\right)_{i=1}^{N}$ are the optimal trading strategies of the minor agents solving (3.1) based on this price process $\left(\varpi_{t}\right)_{t \in[0, T]}$.

We shall show that the resultant equilibrium price process becomes dependent on $\left(\beta_{t}\right)_{t \in[0, T]}$ i.e., we have $\left(\varpi_{t}(\boldsymbol{\beta})\right)_{t \in[0, T]}$. Note that the minor agents do not directly care about $\left(\beta_{t}\right)_{t \in[0, T]}$. They are just destined to face, as price takers, the exogenous market price process, which happens to depend on the major's strategy when it clears the market. The problem of the major agent is now to solve

$$
\inf _{\boldsymbol{\beta} \in \mathbb{A}^{0}} J^{0}(\boldsymbol{\beta})
$$

with the cost functional depending on $f_{0}^{(N)}$ and $g_{0}^{(N)}$ :

$$
J^{0}(\boldsymbol{\beta}):=\mathbb{E}\left[\int_{0}^{T} f_{0}^{(N)}\left(t, X_{t}^{0}, \beta_{t}, \varpi_{t}(\boldsymbol{\beta}), \Lambda_{t}^{0}, c_{t}^{0}\right) \mathrm{d} t+g_{0}^{(N)}\left(X_{T}^{0}, c_{T}^{0}\right)\right]
$$

with her own feedback effects taken into account. $\left(\Lambda_{t}^{0}\right)_{t \in[0, T]}$ is an $\overline{\mathbb{F}}^{0}$-adapted process related to the trading fee to be paid to the exchange. The state dynamics of the major agent describing her position size is assumed to follow

$$
\mathrm{d} X_{t}^{0}=\left(\beta_{t}+l_{0}^{(N)}\left(t, c_{t}^{0}\right)\right) \mathrm{d} t+\sigma_{0}^{(N)}\left(t, c_{t}^{0}\right) \mathrm{d} W_{t}^{0}, \quad t \in[0, T]
$$

with some initial condition $X_{0}^{0} \in \mathbb{R}^{n}$. The superscript $(N)$ of the coefficient functions is added to indicate that there are $(N)$ minor agents. It becomes useful when we take the large- $N$ limit in later sections. We assume that the space of admissible strategies for the major agent is given by $\mathbb{A}^{0}:=\mathbb{H}^{2}\left(\mathbb{F} ; \mathbb{R}^{n}\right) \cap\left\{\beta_{T}=0\right\}$, where the constraint $\beta_{T}=0$ is added in order to forbid the last-time price manipulation.

In our framework, the price process including the feedback effects from the major's action is determined endogenously. This is a clear contrast to the existing literature dealing with the optimal execution strategy, where the form of the price impact as well as the fundamental price process are exogenously given. With appropriate modifications of the cost functions and their interpretations, the current setup may be useful also for economic analysis, for example, the market involving one major producer and a large number of small consumers.

Before going to the details, let us comment on the information structure for the agents.

Remark 3.1. If possible, we naturally want to restrict the space of admissible strategies for each minor agent to $\mathbb{A}^{i}=\mathbb{H}^{2}\left(\mathbb{F}^{i} ; \mathbb{R}^{n}\right), 1 \leq i \leq N$ and that for the major agent to $\mathbb{A}^{0}=\mathbb{H}^{2}\left(\overline{\mathbb{F}}^{0} ; \mathbb{R}^{n}\right) \cap\left\{\beta_{T}=0\right\}$. In other words, we want to realize a market in which each agent only cares about the common market shocks adapted to $\overline{\mathbb{F}}^{0}$ and his/her own idiosyncratic shocks adapted to $\overline{\mathbb{F}}^{i}$. This would be a much plausible model for the real financial market than our setup given above. Unfortunately, this looks impossible in the market consisting of finite number of agents since, in general, the market-clearing price does not solely adapted to $\overline{\mathbb{F}}^{0}$ but is dependent on the idiosyncratic shocks, too.

As already observed in $[27,28]$, we shall see that this ideal situation is actually realized in the large population limit. There, we can restrict the admissible strategy of the $i$ th minor agent to $\mathbb{A}^{i}=\mathbb{H}^{2}\left(\mathbb{F}^{i} ; \mathbb{R}^{n}\right)$, and that of the major agent to $\mathbb{A}^{0}=\mathbb{H}^{2}\left(\overline{\mathbb{F}}^{0} ; \mathbb{R}^{n}\right) \cap\left\{\beta_{T}=0\right\}$. In fact, we can find $\left(\varpi_{t}\right)_{t \in[0, T]}$ is an $\overline{\mathbb{F}}^{0}$-adapted process, i.e. the 
market-clearing price is dependent only on the common market shocks. By the convergence analysis, we shall see that this is approximately true when the population size is large enough.

\subsection{Solving the problem for the minor agents}

Let us solve the problem for each minor agent with given order flow $\left(\beta_{t}\right)_{t \in[0, T]} \in \mathbb{A}^{0}$ of the major agent. This is done in a completely parallel manner with our previous work [28]. We first specify the details of the functions introduced in the last section. For each $1 \leq i \leq N$, we consider the following measurable functions:

$$
\begin{aligned}
\left(l_{i}, \sigma_{i}^{0}, \sigma_{i}\right) & :[0, T] \times \mathbb{R}^{n} \times \mathbb{R}^{n} \ni\left(t, c^{0}, c^{i}\right) \\
& \mapsto\left(l_{i}\left(t, c^{0}, c^{i}\right), \sigma_{i}^{0}\left(t, c^{0}, c^{i}\right), \sigma_{i}\left(t, c^{0}, c^{i}\right)\right) \in\left(\mathbb{R}^{n}, \mathbb{R}^{n \times d_{0}}, \mathbb{R}^{n \times d}\right), \\
\bar{f}_{i}:[0, T] & \times\left(\mathbb{R}^{n}\right)^{4} \ni\left(t, x, \varpi, c^{0}, c^{i}\right) \mapsto \bar{f}_{i}\left(t, x, \varpi, c^{0}, c^{i}\right) \in \mathbb{R}, \\
\bar{g}_{i}:\left(\mathbb{R}^{n}\right)^{3} & \ni\left(x, c^{0}, c^{i}\right) \mapsto \bar{g}_{i}\left(x, c^{0}, c^{i}\right) \in \mathbb{R},
\end{aligned}
$$

as well as $f_{i}:[0, T] \times\left(\mathbb{R}^{n}\right)^{3} \times \mathcal{S}_{+}^{n} \times\left(\mathbb{R}^{n}\right)^{2} \rightarrow \mathbb{R}$ and $g_{i}:\left(\mathbb{R}^{n}\right)^{4} \rightarrow \mathbb{R}$ defined by

$$
\begin{aligned}
& f_{i}\left(t, x, \alpha, \varpi, \Lambda, c^{0}, c^{i}\right):=\langle\varpi, \alpha\rangle+\frac{1}{2}\langle\alpha, \Lambda \alpha\rangle+\bar{f}_{i}\left(t, x, \varpi, c^{0}, c^{i}\right), \\
& g_{i}\left(x, \varpi, c^{0}, c^{i}\right):=-\delta\langle\varpi, x\rangle+\bar{g}_{i}\left(x, c^{0}, c^{i}\right) .
\end{aligned}
$$

Let us explain the economic meaning of the cost functions. By buying (or selling if negative) with speed $\alpha_{t}$, each agent pays (or receives if negative) $\left\langle\alpha_{t}, \varpi_{t}\right\rangle \mathrm{d} t$ amount of cash in the time interval $[t, t+\mathrm{d} t]$. In addition to this direct cost, we suppose that each agent has to pay the service fees to the securities exchange $\frac{1}{2}\left\langle\alpha_{t}, \Lambda \alpha_{t}\right\rangle \mathrm{d} t$ where $\Lambda$ is an $n \times n$ positive definite matrix. These costs are represented by the first two terms of the function $f_{i}$. The first term of $g_{i}$ denotes the mark-to-market value at the closing time with some discount factor $\delta \in[0,1) .{ }^{1}$ The above three terms are assumed to be common across the agents since there is no strong motivation to suppose otherwise. The remaining terms represented by functions $\bar{f}_{i}$ and $\bar{g}_{i}$ can be used to distinguish various characters among the agents. The function $\bar{f}_{i}$ is supposed to represent the running costs which can be dependent on the position size, cash flows, prices of the securities as well as any relevant news available to each agent. The function $\bar{g}_{i}$ puts some penalty on the position size at the terminal time $T$. In particular, we can make the $i$ th agent more risk averse by assigning stronger convexity on $x$ for $\bar{f}_{i}$ and/or $\bar{g}_{i}$.

Example 3.2. Suppose that the $n$ securities have continuous dividend payments $\left(c_{t}^{0}\right)_{t \in[0, T)}$ as well as the rump-sum payment $c_{T}^{0}$ at time $T$. In this case, it may be natural to consider

$$
\begin{aligned}
\bar{f}_{i}\left(t, x, \varpi, c^{0}, c^{i}\right) & =-\left\langle c^{0}, x\right\rangle+\bar{f}_{i}^{\prime}\left(t, x, \varpi, c^{i}\right), \\
\bar{g}_{i}\left(x, \varpi, c^{0}, c^{i}\right) & =-\left\langle c^{0}, x\right\rangle+\bar{g}_{i}^{\prime}\left(x, \varpi, c^{i}\right),
\end{aligned}
$$

with some appropriate measurable functions $\bar{f}_{i}^{\prime}$ and $\bar{g}_{i}^{\prime}$. Here, the first term $\left\langle c^{0}, x\right\rangle$ denotes the benefit from the receipt of the cash flow. The idiosyncratic shock $c^{i}$ can be used in various ways. For example, we may used it to change the risk-averseness with respect to the position size $x$ of each agent according to the arrival of the idiosyncratic information. In fact, this is represented by the functions $c_{i}^{f}\left(\cdot, c^{i}\right)$ and $c_{i}^{g}\left(\cdot, c^{i}\right)$ explained in (iv) of Assumption 3.3 given below.

Let us also introduce the following measurable functions $\left(c_{i}^{f}, c_{i}^{g}, h_{i}^{f}, h_{i}^{g}\right)$ for each $1 \leq i \leq N$ :

$$
\begin{aligned}
& c_{i}^{f}:[0, T] \times\left(\mathbb{R}^{n}\right)^{2} \ni\left(t, c^{0}, c^{i}\right) \mapsto c_{i}^{f}\left(t, c^{0}, c^{i}\right) \in \mathcal{S}_{+}^{n}, \\
& c_{i}^{g}:\left(\mathbb{R}^{n}\right)^{2} \ni\left(c^{0}, c^{i}\right) \mapsto c_{i}^{g}\left(c^{0}, c^{i}\right) \in \mathcal{S}_{+}^{n},
\end{aligned}
$$

\footnotetext{
${ }^{1}$ We shall see that the condition $\delta<1$ is necessary to obtain well-defined terminal condition for the equilibrium.
} 


$$
\begin{aligned}
& h_{i}^{f}:[0, T] \times\left(\mathbb{R}^{n}\right)^{2} \mapsto h_{i}^{f}\left(t, c^{0}, c^{i}\right) \in \mathbb{R}^{n}, \\
& h_{i}^{g}:\left(\mathbb{R}^{n}\right)^{2} \mapsto h_{i}^{g}\left(c^{0}, c^{i}\right) \in \mathbb{R}^{n} .
\end{aligned}
$$

We assume the following conditions:

Assumption 3.3. (Minor-A) Uniformly in $1 \leq i \leq N$, the functions satisfy the followings:

(i) $\left(\Lambda_{t}\right)_{t \in[0, T]}$ is an $\overline{\mathbb{F}}^{0}$-progressively measurable $\mathcal{S}_{+}^{n}$-valued process such that there exist some positive constants $0<\underline{\lambda} \leq \bar{\lambda}<\infty$ satisfying $\underline{\lambda}|\theta|^{2} \leq\left\langle\theta, \Lambda_{t} \theta\right\rangle \leq \bar{\lambda}|\theta|^{2}$ for every $(\omega, t, \theta) \in \Omega \times[0, T] \times \mathbb{R}^{n}$.

(ii) For any $\left(t, c^{0}, c^{i}\right) \in[0, T] \times\left(\mathbb{R}^{n}\right)^{2}$,

$$
\left|l_{i}\left(t, c^{0}, c^{i}\right)\right|+\left|\sigma_{i}^{0}\left(t, c^{0}, c^{i}\right)\right|+\left|\sigma_{i}\left(t, c^{0}, c^{i}\right)\right| \leq L\left(1+\left|c^{0}\right|+\left|c^{i}\right|\right) .
$$

(iii) For any $\left(t, x, \varpi, c^{0}, c^{i}\right) \in[0, T] \times\left(\mathbb{R}^{n}\right)^{4}$,

$$
\left|\bar{f}_{i}\left(t, x, \varpi, c^{0}, c^{i}\right)\right|+\left|\bar{g}_{i}\left(x, c^{0}, c^{i}\right)\right| \leq L\left(1+|x|^{2}+|\varpi|^{2}+\left|c^{0}\right|^{2}+\left|c^{i}\right|^{2}\right) .
$$

(iv) For any $\left(t, x, \varpi, c^{0}, c^{i}\right) \in[0, T] \times\left(\mathbb{R}^{n}\right)^{4}, \bar{f}_{i}$ and $\bar{g}_{i}$ are once continuously differentiable in $x$ with $\varpi$-independent derivatives, and the functions $\partial_{x} \bar{f}_{i}$ and $\partial_{x} \bar{g}_{i}$ have the following affine-form in $x$ :

$$
\begin{aligned}
& \partial_{x} \bar{f}_{i}\left(t, x, \varpi, c^{0}, c^{i}\right)\left(=: \partial_{x} \bar{f}_{i}\left(t, x, c^{0}, c^{i}\right)\right)=c_{i}^{f}\left(t, c^{0}, c^{i}\right) x+h_{i}^{f}\left(t, c^{0}, c^{i}\right), \\
& \partial_{x} \bar{g}_{i}\left(x, c^{0}, c^{i}\right)=c_{i}^{g}\left(c^{0}, c^{i}\right) x+h_{i}^{g}\left(c^{0}, c^{i}\right) .
\end{aligned}
$$

Moreover, the functions $\left(c_{i}^{f}, c_{i}^{g}, h_{i}^{f}, h_{i}^{g}\right)$ satisfy

$$
\begin{aligned}
& \left|h_{i}^{f}\left(t, c^{0}, c^{i}\right)\right|+\left|h_{i}^{g}\left(c^{0}, c^{i}\right)\right| \leq L\left(1+\left|c^{0}\right|+\left|c^{i}\right|\right), \\
& \left|c_{i}^{f}\left(t, c^{0}, c^{i}\right)\right|+\left|c_{i}^{g}\left(c^{0}, c^{i}\right)\right| \leq L, \\
& \left\langle\theta, c_{i}^{f}\left(t, c^{0}, c^{i}\right) \theta\right\rangle \geq \gamma^{f}|\theta|^{2}, \quad\left\langle\theta, c_{i}^{g}\left(c^{0}, c^{i}\right) \theta\right\rangle \geq \gamma^{g}|\theta|^{2}, \quad \forall \theta \in \mathbb{R}^{n},
\end{aligned}
$$

with some positive constants $\gamma^{f}, \gamma^{g}>0$.

This is a special situation studied in Section 3.1 of [28]. In fact, the conditions in Assumption (Minor-A) are significantly more stringent than those used in [28]. We do this in order to avoid introducing many sets of assumptions incrementally in later sections. In particular, the affine-form condition in (iv) is to be used when we verify the optimality condition for the major agent based on Theorem A.3. The associated (reduced) Hamiltonian for the $i$ th agent $H_{i}:[0, T] \times\left(\mathbb{R}^{n}\right)^{4} \times \mathcal{S}_{+}^{n} \times\left(\mathbb{R}^{n}\right)^{2} \rightarrow \mathbb{R}$ is given by

$$
H_{i}\left(t, x, y, \alpha, \varpi, \Lambda, c^{0}, c^{i}\right):=\left\langle y, \alpha+l_{i}\left(t, c^{0}, c^{i}\right)\right\rangle+f_{i}\left(t, x, \alpha, \varpi, \Lambda, c^{0}, c^{i}\right)
$$

which is jointly convex in $(x, y, \alpha)$ and strictly so in $(x, \alpha)$. The unique minimizer $\alpha$ of $H_{i}$ is given by

$$
\widehat{\alpha}(y, \varpi):=-\bar{\Lambda}(y+\varpi),
$$

with $\bar{\Lambda}:=\Lambda^{-1}$. Therefore, the adjoint equation associated with the problem (3.1) for the $i$ th agent arising from the stochastic maximum principle is given by, for $t \in[0, T]$,

$$
\left\{\begin{array}{l}
\mathrm{d} X_{t}^{i}=\left(-\bar{\Lambda}_{t}\left(Y_{t}^{i}+\varpi_{t}\right)+l_{i}\left(t, c_{t}^{0}, c_{t}^{i}\right)\right) \mathrm{d} t+\sigma_{i}^{0}\left(t, c_{t}^{0}, c_{t}^{i}\right) \mathrm{d} W_{t}^{0}+\sigma_{i}\left(t, c_{t}^{0}, c_{t}^{i}\right) \mathrm{d} W_{t}^{i}, \\
d Y_{t}^{i}=-\partial_{x} \bar{f}_{i}\left(t, X_{t}^{i}, c_{t}^{0}, c_{t}^{i}\right) \mathrm{d} t+Z_{t}^{i, 0} \mathrm{~d} W_{t}^{0}+\sum_{j=1}^{N} Z_{t}^{i, j} \mathrm{~d} W_{t}^{j},
\end{array}\right.
$$


with $X_{0}^{i}=\xi^{i}$ and $Y_{T}^{i}=-\delta \varpi_{T}+\partial_{x} \bar{g}_{i}\left(X_{T}^{i}, c_{T}^{0}, c_{T}^{i}\right)$.

Theorem 3.4. Let Assumption (Minor-A) be in force. Then, for any $\left(\varpi_{t}\right)_{t \in[0, T]} \in \mathbb{H}^{2}\left(\mathbb{F} ; \mathbb{R}^{n}\right)$ satisfying $\varpi_{T} \in$ $\mathbb{L}^{2}\left(\mathcal{F}_{T} ; \mathbb{R}^{n}\right)$, the problem (3.1) for each agent $1 \leq i \leq N$ is uniquely characterized by the FBSDE (3.5) which is strongly solvable with a unique solution $\left(X^{i}, Y^{i}, Z^{i, 0},\left(Z^{i, j}\right)_{j=1}^{N}\right) \in \mathbb{S}^{2}\left(\mathbb{F} ; \mathbb{R}^{n}\right) \times \mathbb{S}^{2}\left(\mathbb{F} ; \mathbb{R}^{n}\right) \times \mathbb{H}^{2}\left(\mathbb{F} ; \mathbb{R}^{n \times d_{0}}\right) \times$ $\left(\mathbb{H}^{2}\left(\mathbb{F} ; \mathbb{R}^{n \times d}\right)\right)^{N}$.

Proof. This is the direct result of Theorem 3.1 in [28]. One can easily check Assumption 3.1 in [28] is satisfied under (Minor-A). Although $\left(\Lambda_{t}\right)_{t \in[0, T]}$ is now stochastic, it does not introduce any additional difficulty. The existence of the unique solution to the FBSDE (3.5) can also be proved by the direct application of Theorem 2.6 in [49] (with $\beta_{1}, \mu_{1}>0$ ), which is repeatedly used in the following sections.

\subsection{Deriving the equilibrium price process for a given $\left(\beta_{t}\right)_{t \in[0, T]}$}

From Theorem 3.4, we find that the optimal trading speed of each minor agent $1 \leq i \leq N$ is given by

$$
\widehat{\alpha}_{t}^{i}=-\bar{\Lambda}_{t}\left(Y_{t}^{i}+\varpi_{t}\right), \quad t \in[0, T]
$$

for any exogenous input $\left(\varpi_{t}\right)_{t \in[0, T]}$. Since the market-clearing condition requires $\sum_{i=1}^{N} \widehat{\alpha}_{t}^{i}+\beta_{t}=0$, d $t \otimes d \mathbb{P}$-a.e. the market price process needs to satisfy

$$
\varpi_{t}=-\mathfrak{m}\left(\left(Y_{t}\right)\right)+\Lambda_{t} \frac{\beta_{t}}{N}, \quad t \in[0, T]
$$

This relation suggests a large system of fully-coupled FBSDEs given below: for $1 \leq i \leq N$,

$$
\left\{\begin{array}{l}
\mathrm{d} X_{t}^{i}=\left\{-\bar{\Lambda}_{t}\left(Y_{t}^{i}-\mathfrak{m}\left(\left(Y_{t}\right)\right)\right)-\frac{\beta_{t}}{N}+l_{i}\left(t, c_{t}^{0}, c_{t}^{i}\right)\right\} \mathrm{d} t+\sigma_{i}^{0}\left(t, c_{t}^{0}, c_{t}^{i}\right) \mathrm{d} W_{t}^{0}+\sigma_{i}\left(t, c_{t}^{0}, c_{t}^{i}\right) \mathrm{d} W_{t}^{i}, \\
\mathrm{~d} Y_{t}^{i}=-\partial_{x} \bar{f}_{i}\left(t, X_{t}^{i}, c_{t}^{0}, c_{t}^{i}\right) \mathrm{d} t+Z_{t}^{i, 0} \mathrm{~d} W_{t}^{0}+\sum_{j=1}^{N} Z_{t}^{i, j} \mathrm{~d} W_{t}^{j},
\end{array}\right.
$$

with

$$
\left\{\begin{array}{l}
X_{0}^{i}=\xi^{i} \\
Y_{T}^{i}=\frac{\delta}{1-\delta} \mathfrak{m}\left(\left(c_{j}^{g}\left(c_{T}^{0}, c_{T}^{j}\right) X_{T}^{j}+h_{j}^{g}\left(c_{T}^{0}, c_{T}^{j}\right)\right)_{j=1}^{N}\right)+c_{i}^{g}\left(c_{T}^{0}, c_{T}^{i}\right) X_{T}^{i}+h_{i}^{g}\left(c_{T}^{0}, c_{T}^{i}\right) .
\end{array}\right.
$$

The terminal condition for $Y^{i}$ is implied from

$$
Y_{T}^{i}=-\delta \varpi_{T}+\partial_{x} \bar{g}_{i}\left(X_{T}^{i}, c_{T}^{0}, c_{T}^{i}\right)
$$

and the fact that $\varpi_{T}=-\mathfrak{m}\left(\left(Y_{T}\right)\right.$ ) (note that $\beta_{T}=0$ ). We have the following result.

Theorem 3.5. Let Assumption (Minor-A) be in force. With a given strategy $\left(\beta_{t}\right)_{t \in[0, T]} \in \mathbb{A}^{0}$ of the major agent, the market-clearing equilibrium with a square integrable price process $\left(\varpi_{t}\right)_{t \in[0, T]} \in \mathbb{H}^{2}\left(\mathbb{F} ; \mathbb{R}^{n}\right)$ with $\varpi_{T} \in \mathbb{L}^{2}\left(\mathcal{F}_{T} ; \mathbb{R}^{n}\right)$ exists if and only if there exists a solution $\left(X^{i}, Y^{i}, Z^{i, 0},\left(Z^{i, j}\right)_{j=1}^{N}\right) \in \mathbb{S}^{2}\left(\mathbb{F} ; \mathbb{R}^{n}\right) \times \mathbb{S}^{2}\left(\mathbb{F} ; \mathbb{R}^{n}\right) \times$ $\mathbb{H}^{2}\left(\mathbb{F} ; \mathbb{R}^{n \times d_{0}}\right) \times\left(\mathbb{H}^{2}\left(\mathbb{F} ; \mathbb{R}^{n \times d}\right)\right)^{N}, 1 \leq i \leq N$ to the $N$-coupled system of FBSDEs (3.7) with (3.8).

Proof. This is a simple modification of Theorem 3.2 in [28]. The necessity is obvious from Theorem 3.4 and the above discussion. On the other hand, suppose that there exists a square integrable solution to the $N$-coupled FBSDEs (3.7) with (3.8). Let us define the price process $\varpi$ by (3.6) using the solution $\left(Y^{i}\right)_{i=1}^{N}$. Then, with this $\varpi$ as an input, the solution $\left(y_{t}^{i}\right)_{t \in[0, T]}$ to $(3.5)$, which corresponds to the problem for the $i$ th agent, actually satisfies $y^{i}=Y^{i}$ in $\mathbb{S}^{2}\left(\mathbb{F} ; \mathbb{R}^{n}\right)$ due to the uniqueness of the solution to $(3.5)$. Therefore, the market-clearing condition is satisfied. 
Assumption 3.6. (Minor-B)

There exists some $\mathcal{F}_{T}$-measurable $\mathcal{S}^{n}$-valued random variable $\mathfrak{c}$ such that

$$
\mathfrak{a}:=\frac{\delta}{1-\delta}\left\|\mathfrak{c}-c_{i}^{g}\left(c_{T}^{0}, c_{T}^{i}\right)\right\|_{\infty}<\gamma^{g}, \quad 1 \leq i \leq N
$$

Theorem 3.7. Let Assumptions (Minor-A, B) be in force. Then, for any given $\left(\beta_{t}\right)_{t \in[0, T]} \in \mathbb{A}^{0}$, the $N$ coupled system of FBSDEs (3.7) with (3.8) has a unique strong solution $\left(X^{i}, Y^{i}, Z^{i, 0},\left(Z^{i, j}\right)_{j=1}^{N}\right) \in \mathbb{S}^{2}\left(\mathbb{F} ; \mathbb{R}^{n}\right) \times$ $\mathbb{S}^{2}\left(\mathbb{F} ; \mathbb{R}^{n}\right) \times \mathbb{H}^{2}\left(\mathbb{F} ; \mathbb{R}^{n \times d_{0}}\right) \times\left(\mathbb{H}^{2}\left(\mathbb{F} ; \mathbb{R}^{n \times d}\right)\right)^{N}, 1 \leq i \leq N$.

Proof. Let $x^{i}, y^{i} \in \mathbb{R}^{n}$ be arbitrary constants. For notational simplicity, we write $x=\left(x^{i}\right)_{i=1}^{N}$ and $y=\left(y^{i}\right)_{i=1}^{N}$. Put

$$
\begin{aligned}
& \operatorname{drift}\left[x^{i}\right](t, y):=-\bar{\Lambda}_{t}\left(y^{i}-\mathfrak{m}((y))\right)-\frac{\beta_{t}}{N}+l_{i}\left(t, c_{t}^{0}, c_{t}^{i}\right), \\
& \operatorname{drift}\left[y^{i}\right](t, x):=-\partial_{x} \bar{f}_{i}\left(t, x^{i}, c_{t}^{0}, c_{t}^{i}\right), \\
& \operatorname{terminal}\left[y^{i}\right](x):=\frac{\delta}{1-\delta} \mathfrak{m}\left(\left(c_{j}^{g}\left(c_{T}^{0}, c_{T}^{j}\right) x^{j}+h_{j}^{g}\left(c_{T}^{0}, c_{T}^{j}\right)\right)_{j=1}^{N}\right)+c_{i}^{g}\left(c_{T}^{0}, c_{T}^{i}\right) x^{i}+h_{i}^{g}\left(c_{T}^{0}, c_{T}^{i}\right) .
\end{aligned}
$$

For two inputs $(x, y)$ and $\left(x^{\prime}, y^{\prime}\right)$, with the conventions $\Delta x^{i}:=x^{i}-x^{i \prime}, \Delta y^{i}:=y^{i}-y^{i \prime}$,

$$
\begin{aligned}
& \Delta \operatorname{drift}\left[x^{i}\right](t):=\operatorname{drift}\left[x^{i}\right](t, y)-\operatorname{drift}\left[x^{i}\right]\left(t, y^{\prime}\right), \\
& \Delta \operatorname{drift}\left[y^{i}\right](t):=\operatorname{drift}\left[y^{i}\right](t, x)-\operatorname{drift}\left[y^{i}\right]\left(t, x^{\prime}\right), \\
& \Delta \operatorname{terminal}\left[y^{i}\right]:=\operatorname{terminal}\left[y^{i}\right](x)-\operatorname{terminal}\left[y^{i}\right]\left(x^{\prime}\right),
\end{aligned}
$$

we have

$$
\begin{aligned}
& \sum_{i=1}^{N}\left\langle\Delta \operatorname{drift}\left[x^{i}\right](t), \Delta y^{i}\right\rangle=-\sum_{i=1}^{N}\left\langle\bar{\Lambda}_{t} \Delta y^{i}, \Delta y^{i}\right\rangle+N\left\langle\bar{\Lambda}_{t} \mathfrak{m}((\Delta y)), \mathfrak{m}((\Delta y))\right\rangle \leq 0, \\
& \sum_{i=1}^{N}\left\langle\Delta \operatorname{drift}\left[y^{i}\right](t), \Delta x^{i}\right\rangle=-\left\langle c_{i}^{f}\left(t, c_{t}^{0}, c_{t}^{i}\right) \Delta x^{i}, \Delta x^{i}\right\rangle \leq-\gamma^{f} \sum_{i=1}^{N}\left|\Delta x^{i}\right|^{2},
\end{aligned}
$$

$$
\begin{aligned}
& \sum_{i=1}^{N}\left\langle\Delta \text { terminal }\left[y^{i}\right], \Delta x^{i}\right\rangle \\
& \quad=\frac{\delta N}{1-\delta}\left\langle\mathfrak{m}\left(\left(c_{i}^{g}\left(c_{T}^{0}, c_{T}^{i}\right) \Delta x^{i}\right)_{i=1}^{N}\right), \mathfrak{m}((\Delta x))\right\rangle+\sum_{i=1}^{N}\left\langle c_{i}^{g}\left(c_{T}^{0}, c_{T}^{i}\right) \Delta x^{i}, \Delta x^{i}\right\rangle \\
& \quad \geq \frac{\delta N}{1-\delta}\langle\mathfrak{c} \mathfrak{m}((\Delta x)), \mathfrak{m}((\Delta x))\rangle+\left(\gamma^{g}-\mathfrak{a}\right) \sum_{i=1}^{N}\left|\Delta x^{i}\right|^{2} \geq\left(\gamma^{g}-\mathfrak{a}\right) \sum_{i=1}^{N}\left|\Delta x^{i}\right|^{2}
\end{aligned}
$$

Thus we can apply Theorem 2.6 in [49] with $\left(\beta_{1}, \mu_{1}\right)=\left(\gamma^{f}, \gamma^{g}-\mathfrak{a}\right)$ and $G=I$. See also the proof for Theorem 3.3 in [28], which can be applied in essentially the same way for the current problem. 


\subsection{Optimization problem for the major agent}

We now investigate the optimization problem for the major agent. From Theorems 3.5 and 3.7, her problem is given by $\inf _{\boldsymbol{\beta} \in \mathbb{A}^{0}} J^{0}(\boldsymbol{\beta})$ with

$$
J^{0}(\boldsymbol{\beta}):=\mathbb{E}\left[\int_{0}^{T} f_{0}^{(N)}\left(t, X_{t}^{0}, \beta_{t},-\mathfrak{m}\left(\left(Y_{t}\right)\right)+\Lambda_{t} \frac{\beta_{t}}{N}, \Lambda_{t}^{0}, c_{t}^{0}\right) \mathrm{d} t+g_{0}^{(N)}\left(X_{T}^{0}, c_{T}^{0}\right)\right],
$$

subject to the dynamic constraints with $1 \leq i \leq N$ :

$$
\left\{\begin{array}{l}
\mathrm{d} X_{t}^{0}=\left(\beta_{t}+l_{0}^{(N)}\left(t, c_{t}^{0}\right)\right) \mathrm{d} t+\sigma_{0}^{(N)}\left(t, c_{t}^{0}\right) \mathrm{d} W_{t}^{0}, \\
\mathrm{~d} X_{t}^{i}=\left\{-\bar{\Lambda}_{t}\left(Y_{t}^{i}-\mathfrak{m}\left(\left(Y_{t}\right)\right)\right)-\frac{\beta_{t}}{N}+l_{i}\left(t, c_{t}^{0}, c_{t}^{i}\right)\right\} \mathrm{d} t+\sigma_{i}^{0}\left(t, c_{t}^{0}, c_{t}^{i}\right) \mathrm{d} W_{t}^{0}+\sigma_{i}\left(t, c_{t}^{0}, c_{t}^{i}\right) \mathrm{d} W_{t}^{i}, \\
\mathrm{~d} Y_{t}^{i}=-\partial_{x} \bar{f}_{i}\left(t, X_{t}^{i}, c_{t}^{0}, c_{t}^{i}\right) \mathrm{d} t+Z_{t}^{i, 0} \mathrm{~d} W_{t}^{0}+\sum_{j=1}^{N} Z_{t}^{i, j} \mathrm{~d} W_{t}^{j}, \quad t \in[0, T]
\end{array}\right.
$$

with

$$
\left\{\begin{array}{l}
X_{0}^{0}=N \chi^{0}, \quad \chi^{0} \in \mathbb{R}^{n}, \\
X_{0}^{i}=\xi^{i} \\
Y_{T}^{i}=\frac{\delta}{1-\delta} \mathfrak{m}\left(\left(c_{j}^{g}\left(c_{T}^{0}, c_{T}^{j}\right) X_{T}^{j}+h_{j}^{g}\left(c_{T}^{0}, c_{T}^{j}\right)\right)_{j=1}^{N}\right)+c_{i}^{g}\left(c_{T}^{0}, c_{T}^{i}\right) X_{T}^{i}+h_{i}^{g}\left(c_{T}^{0}, c_{T}^{i}\right) .
\end{array}\right.
$$

As we can see, the problem for the major agent turns out to be an optimization with respect to the system of controlled-FBSDEs instead of controlled-SDEs. See, for relevant information, Appendix A and the references therein.

Remark 3.8. At first glance, it may seem to be a linear price impact model popular in the literature dealing with the optimal execution problem. However, notice that the term $-\mathfrak{m}\left(\left(Y_{t}^{i}\right)\right)$ is also dependent on the major agent's strategy in a complicated fashion.

Since we want to study the large population limit $N \rightarrow \infty$ in later sections, it is convenient to define the normalized measurable functions:

$$
\begin{aligned}
& \left(\mathfrak{l}_{0}, \mathfrak{s}_{0}\right):[0, T] \times \mathbb{R}^{n} \ni\left(t, c^{0}\right) \mapsto\left(\mathfrak{l}_{0}\left(t, c^{0}\right), \mathfrak{s}_{0}\left(t, c^{0}\right)\right) \in\left(\mathbb{R}^{n}, \mathbb{R}^{n \times d_{0}}\right), \\
& \overline{\mathfrak{f}}_{0}:[0, T] \times\left(\mathbb{R}^{n}\right)^{2} \ni\left(t, x, c^{0}\right) \mapsto \overline{\mathfrak{f}}_{0}\left(t, x, c^{0}\right) \in \mathbb{R}, \\
& \mathfrak{g}_{0}:\left(\mathbb{R}^{n}\right)^{2} \ni\left(x, c^{0}\right) \mapsto \mathfrak{g}_{0}\left(x, c^{0}\right) \in \mathbb{R}
\end{aligned}
$$

and $\mathfrak{f}_{0}:[0, T] \times\left(\mathbb{R}^{n}\right)^{3} \times \mathcal{S}^{n} \times \mathbb{R}^{n} \rightarrow \mathbb{R}$ by

$$
\mathfrak{f}_{0}\left(t, x, \beta, \varpi, \Lambda^{0}, c^{0}\right):=\langle\beta, \varpi\rangle+\frac{1}{2}\left\langle\beta, \Lambda^{0} \beta\right\rangle+\overline{\mathfrak{f}}_{0}\left(t, x, c^{0}\right) .
$$

We then define the unnormalized functions by

$$
\begin{aligned}
& l_{0}^{(N)}\left(t, c^{0}\right):=N \mathfrak{l}_{0}\left(t, c^{0}\right), \\
& \sigma_{0}^{(N)}\left(t, c^{0}\right):=N \mathfrak{s}_{0}\left(t, c^{0}\right), \\
& f_{0}^{(N)}\left(t, x, \beta, \varpi, \Lambda^{0}, c^{0}\right):=N \mathfrak{f}_{0}\left(t, x / N, \beta / N, \varpi, \Lambda^{0}, c^{0}\right), \\
& \bar{f}_{0}^{(N)}\left(t, x, c^{0}\right):=N \overline{\mathfrak{f}}_{0}\left(t, x / N, c^{0}\right), \\
& g_{0}^{(N)}\left(x, c^{0}\right):=N \mathfrak{g}_{0}\left(x / N, c^{0}\right) .
\end{aligned}
$$


Note that, we have

$$
f_{0}^{(N)}\left(t, x, \beta, \varpi, \Lambda^{0}, c^{0}\right)=\langle\beta, \varpi\rangle+\frac{1}{2}\left\langle\beta, \frac{\Lambda^{0}}{N} \beta\right\rangle+\bar{f}_{0}^{(N)}\left(t, x, c^{0}\right) .
$$

Economic meaning of each term can be understood in the same way as the one for minor agents given in Section 3.2.

Let us introduce the following assumptions.

Assumption 3.9. (Major)

(i) $\left(\Lambda_{t}^{0}\right)_{t \in[0, T]}$ is an $\overline{\mathbb{F}}^{0}$-progressively measurable $\mathcal{S}^{n}$-valued process such that there exist some positive constants $0<\underline{\lambda} \leq \bar{\lambda}<\infty$ satisfying $\underline{\lambda}|\theta|^{2} \leq\left\langle\theta,\left(\Lambda_{t}^{0}+2 \Lambda_{t}\right) \theta\right\rangle \leq \bar{\lambda}|\theta|^{2}$ for every $(\omega, t, \theta) \in[0, T] \times \Omega \times \mathbb{R}^{n}$.

(ii) For any $\left(t, c^{0}\right) \in[0, T] \times \mathbb{R}^{n},\left|\mathfrak{l}_{0}\left(t, c^{0}\right)\right|+\left|\mathfrak{s}_{0}\left(t, c^{0}\right)\right| \leq L_{0}\left(1+\left|c^{0}\right|\right)$.

(iii) For any $\left(t, x^{0}, c^{0}\right) \in[0, T] \times\left(\mathbb{R}^{n}\right)^{2}$,

$$
\left|\overline{\mathfrak{f}}_{0}\left(t, x^{0}, c^{0}\right)\right|+\left|\mathfrak{g}_{0}\left(x^{0}, c^{0}\right)\right| \leq L_{0}\left(1+\left|x^{0}\right|^{2}+\left|c^{0}\right|^{2}\right) .
$$

(iv) $\overline{\mathfrak{f}}_{0}$ and $\mathfrak{g}_{0}$ are once continuously differentiable in $x$ and satisfy

$$
\begin{aligned}
& \left|\partial_{x} \overline{\mathfrak{f}}_{0}\left(t, x^{0}, c^{0}\right)\right|+\left|\partial_{x} \mathfrak{g}_{0}\left(x^{0}, c^{0}\right)\right| \leq L_{0}\left(1+\left|x^{0}\right|+\left|c^{0}\right|\right), \\
& \left|\partial_{x} \overline{\mathfrak{f}}_{0}\left(t, x^{0 \prime}, c^{0}\right)-\partial_{x} \overline{\mathfrak{f}}_{0}\left(t, x^{0}, c^{0}\right)\right|+\left|\partial_{x} \mathfrak{g}_{0}\left(x^{0 \prime}, c^{0}\right)-\partial_{x} \mathfrak{g}_{0}\left(x^{0}, c^{0}\right)\right| \leq L_{0}\left|x^{0 \prime}-x^{0}\right|,
\end{aligned}
$$

for any $\left(t, x^{0}, x^{0 \prime}, c^{0}\right) \in[0, T] \times\left(\mathbb{R}^{n}\right)^{3}$.

(v) $\overline{\mathfrak{f}}_{0}$ and $\mathfrak{g}_{0}$ are strictly convex in the sense that there exist some positive constants $\gamma_{0}^{f}, \gamma_{0}^{g}>0$ and

$$
\begin{aligned}
& \overline{\mathfrak{f}}_{0}\left(t, x^{0 \prime}, c^{0}\right)-\overline{\mathfrak{f}}_{0}\left(t, x^{0}, c^{0}\right)-\left\langle x^{0 \prime}-x^{0}, \partial_{x} \overline{\mathfrak{f}}_{0}\left(t, x^{0}, c^{0}\right)\right\rangle \geq \frac{\gamma_{0}^{f}}{2}\left|x^{0 \prime}-x^{0}\right|^{2}, \\
& \mathfrak{g}_{0}\left(x^{0 \prime}, c^{0}\right)-\mathfrak{g}_{0}\left(x^{0}, c^{0}\right)-\left\langle x^{0 \prime}-x^{0}, \partial_{x} \mathfrak{g}_{0}\left(x^{0}, c^{0}\right)\right\rangle \geq \frac{\gamma_{0}^{g}}{2}\left|x^{0 \prime}-x^{0}\right|^{2},
\end{aligned}
$$

hold for any $\left(t, x^{0}, x^{0 \prime}, c^{0}\right) \in[0, T] \times\left(\mathbb{R}^{n}\right)^{3}$.

For later use, let us put

$$
\gamma_{0}^{f(N)}:=\frac{\gamma_{0}^{f}}{N}, \quad \gamma_{0}^{g(N)}:=\frac{\gamma_{0}^{g}}{N}
$$

Remark 3.10. With the above definition, we have

$$
\begin{aligned}
\partial_{x} \bar{f}_{0}^{(N)}\left(t, x, c^{0}\right) & =N \frac{\partial}{\partial x} \overline{\mathfrak{f}}_{0}\left(t, x / N, c^{0}\right) \\
& =N \frac{\partial(x / N)}{\partial x} \partial_{x} \overline{\mathfrak{f}}_{0}\left(t, x / N, c^{0}\right)=\partial_{x} \overline{\mathfrak{f}}_{0}\left(t, x / N, c^{0}\right) .
\end{aligned}
$$

and similar relation for $\partial_{x} \mathfrak{g}_{0}$.

Remark 3.11. For the analysis with a fixed $N$, such a scaling is arbitrary and irrelevant. However, it plays an important role when we study the large population limit $N \rightarrow \infty$. In particular, the market share of the major agent must grow proportionally to the population size $N$. For example, if the cost functions contain $\left\langle\beta, \Lambda^{0} \beta\right\rangle$ instead of $\left\langle\beta, \frac{\Lambda^{0}}{N} \beta\right\rangle$, the market share of the major agent becomes negligible in the large population limit. In this case, we obtain the same market price as in $[27,28]$. 
Following the analysis done in Appendix A, let us introduce the adjoint variables $\left(p^{0},\left(p^{i}\right)_{i=1}^{N},\left(r^{i}\right)_{i=1}^{N}\right)$ for $\left(x^{0},\left(x^{i}\right)_{i=1}^{N},\left(y^{i}\right)_{i=1}^{N}\right)$, respectively. The (reduced) Hamiltonian

$$
\mathcal{H}:[0, T] \times \mathbb{R}^{n} \times\left(\mathbb{R}^{n}\right)^{N} \times\left(\mathbb{R}^{n}\right)^{N} \times \mathbb{R}^{n} \times\left(\mathbb{R}^{n}\right)^{N} \times\left(\mathbb{R}^{n}\right)^{N} \times \mathbb{R}^{n} \times \mathcal{S}^{n} \times \mathcal{S}_{+}^{n} \times \mathbb{R}^{n} \times\left(\mathbb{R}^{n}\right)^{N} \rightarrow \mathbb{R}
$$

of the system is defined by

$$
\begin{aligned}
\mathcal{H}( & \left.t, x^{0},\left(x^{i}\right)_{i=1}^{N},\left(y^{i}\right)_{i=1}^{N}, p^{0},\left(p^{i}\right)_{i=1}^{N},\left(r^{i}\right)_{i=1}^{N}, \beta, \Lambda^{0}, \Lambda, c^{0},\left(c^{i}\right)_{i=1}^{N}\right) \\
:= & \left\langle p^{0}, \beta+l_{0}^{(N)}\left(t, c^{0}\right)\right\rangle+\sum_{i=1}^{N}\left\langle p^{i},-\bar{\Lambda}\left(y^{i}-\mathfrak{m}((y))\right)-\frac{\beta}{N}+l_{i}\left(t, c^{0}, c^{i}\right)\right\rangle \\
& +\sum_{i=1}^{N}\left\langle r^{i},-\partial_{x} \bar{f}_{i}\left(t, x^{i}, c^{0}, c^{i}\right)\right\rangle \\
& +\left\langle\beta,-\mathfrak{m}((y))+\Lambda \frac{\beta}{N}\right\rangle+\frac{1}{2}\left\langle\beta, \frac{\Lambda^{0}}{N} \beta\right\rangle+\bar{f}_{0}^{(N)}\left(t, x^{0}, c^{0}\right) .
\end{aligned}
$$

For a given set of $p^{0},\left(p^{i}\right)_{i=1}^{N},\left(r^{i}\right)_{i=1}^{N}$ (and also $\left(\Lambda^{0}, \Lambda, c^{0},\left(c^{i}\right)_{i=1}^{N}\right)$ ), it is straightforward to check that $\mathcal{H}$ is jointly convex in $\left(x^{0},\left(x^{i}\right)_{i=1}^{N},\left(y^{i}\right)_{i=1}^{N}, \beta\right)$ and strictly convex in $\left(x^{0}, \beta\right)$. Here, recall that $\partial_{x} \bar{f}_{i}$ is affine in $x^{i}$ by Assumption (Minor-A, (iv)). For given inputs, the minimizer of the Hamiltonian $\widehat{\beta}:=\operatorname{argmin} \mathcal{H}(\beta)$ is given by

$$
\widehat{\beta}=N \overline{\mathcal{V}}^{0}\left(-p^{0}+\mathfrak{m}((y))+\mathfrak{m}((p))\right)
$$

where $\overline{\mathcal{V}}^{0}:=\left(\Lambda^{0}+2 \Lambda\right)^{-1}$.

The adjoint equations for $\left(p^{0},\left(p^{i}\right)_{i=1}^{N},\left(r^{i}\right)_{i=1}^{N}\right)$ can be found from (A.2): for $1 \leq i \leq N$,

$$
\left\{\begin{array}{l}
\mathrm{d} P_{t}^{0}=-\partial_{x} \bar{f}_{0}^{(N)}\left(t, X_{t}^{0}, c_{t}^{0}\right) \mathrm{d} t+Q_{t}^{0,0} \mathrm{~d} W_{t}^{0}+\sum_{j=1}^{N} Q_{t}^{0, j} \mathrm{~d} W_{t}^{j}, \\
\mathrm{~d} P_{t}^{i}=c_{i}^{f}\left(t, c_{t}^{0}, c_{t}^{i}\right) R_{t}^{i} d t+Q_{t}^{i, 0} \mathrm{~d} W_{t}^{0}+\sum_{j=1}^{N} Q_{t}^{i, j} \mathrm{~d} W_{t}^{j}, \\
\mathrm{~d} R_{t}^{i}=\left\{\bar{\Lambda}_{t}\left(P_{t}^{i}-\mathfrak{m}\left(\left(P_{t}\right)\right)\right)+\frac{\beta_{t}}{N}\right\} \mathrm{d} t,
\end{array}\right.
$$

with

$$
\left\{\begin{array}{l}
P_{T}^{0}=\partial_{x} g_{0}^{(N)}\left(X_{T}^{0}, c_{T}^{0}\right) \\
P_{T}^{i}=-c_{i}^{g}\left(c_{T}^{0}, c_{T}^{i}\right)\left(R_{T}^{i}+\frac{\delta}{1-\delta} \mathfrak{m}\left(\left(R_{T}\right)\right)\right) \\
R_{0}^{i}=0
\end{array}\right.
$$

Note that the forward and backward processes $x$ and $y$ in (A.1) correspond to $\left(X^{0},\left(X^{i}\right)_{i=1}^{N}\right)$ and $\left(Y^{i}\right)_{i=1}^{N}$ in (3.10), respectively. As for the adjoint processes, $p$ and $r$ in (A.2) corresponds to $\left(P^{0},\left(P^{i}\right)_{i=1}^{N}\right)$ and $\left(R^{i}\right)_{i=1}^{N}$, respectively. By checking Assumption A.1 using the above relations, we obtain the next theorem.

Theorem 3.12. Let Assumptions (Minior-A, B) and (Major) be in force. Suppose that the system of FBSDEs (3.10) and (3.15) with boundary conditions (3.11) and (3.16) has a solution $X^{0}, Y^{i}, P^{0}, P^{i}, R^{i} \in \mathbb{S}^{2}\left(\mathbb{F} ; \mathbb{R}^{n}\right)$, $Z^{i, 0}, Q^{0,0}, Q^{i, 0} \in \mathbb{H}^{2}\left(\mathbb{F} ; \mathbb{R}^{n \times d_{0}}\right)$, and $Z^{i, j}, Q^{0, j}, Q^{i, j} \in \mathbb{H}^{2}\left(\mathbb{F} ; \mathbb{R}^{n \times d}\right), 1 \leq i, j \leq N$, with the control process $\beta_{t}=$ $\widehat{\beta}_{t}, t \in[0, T)$ i.e.,

$$
\widehat{\beta}_{t}=N \overline{\mathcal{V}}_{t}^{0}\left(-P_{t}^{0}+\mathfrak{m}\left(\left(Y_{t}\right)\right)+\mathfrak{m}\left(\left(P_{t}\right)\right)\right), \quad \overline{\mathcal{V}}_{t}^{0}:=\left(\Lambda_{t}^{0}+2 \Lambda_{t}\right)^{-1}
$$


Then, $\left(\widehat{\beta}_{t}\right)_{t \in[0, T)}$ (with $\left.\widehat{\beta}_{T}=0\right)$ is the unique optimal control for the major agent.

Proof. This is the direct result of Theorem A.3. Note that Assumption (Minor-A) (iv) plays a crucial role to guarantee the joint convexity of $\mathcal{H}$ and the affine property of $\Phi$ required in the theorem.

Remark 3.13 (on the condition $\beta_{T}=0$ ). In the current work, we restrict the admissible strategies of the major agent to $\left\{\beta_{T}=0\right\}$. Since $\{t=T\}$ is the Lebesgue null set, $\beta_{T}$ does not affect the terminal position size $X_{T}^{0}$ of the major agent. Nevertheless, it affects the equilibrium price at time $T$ by the relation (3.6). Therefore, in general, the major agent has an incentive to manipulate the price by changing $\beta_{T}$. In order to make the optimization problem at $T$ well-defined, we need a strict convexity in the terminal cost with respect to $\varpi_{T}$ after including complicated feedback effects from the minor agents. Since this makes the analysis intractable for us, we restrict to $\left\{\beta_{T}=0\right\}$ and also make $g_{0}^{(N)}$ independent from $\varpi_{T}$ at the moment. We leave this interesting problem on general $\beta_{T}$ at the terminal time for future research.

\subsection{Existence of the optimal solution for the major agent}

From Theorem 3.12, the crucial target of our analysis is the following coupled system of FBSDEs:

$$
\left\{\begin{array}{l}
\mathrm{d} X_{t}^{0}=\left(\widehat{\beta}_{t}+l_{0}^{(N)}\left(t, c_{t}^{0}\right)\right) \mathrm{d} t+\sigma_{0}^{(N)}\left(t, c_{t}^{0}\right) \mathrm{d} W_{t}^{0}, \\
\mathrm{~d} X_{t}^{i}=\left\{-\bar{\Lambda}_{t}\left(Y_{t}^{i}-\mathfrak{m}\left(\left(Y_{t}\right)\right)\right)-\frac{\widehat{\beta}_{t}}{N}+l_{i}\left(t, c_{t}^{0}, c_{t}^{i}\right)\right\} \mathrm{d} t+\sigma_{i}^{0}\left(t, c_{t}^{0}, c_{t}^{i}\right) \mathrm{d} W_{t}^{0}+\sigma_{i}\left(t, c_{t}^{0}, c_{t}^{i}\right) \mathrm{d} W_{t}^{i} \\
\mathrm{~d} R_{t}^{i}=\left\{\bar{\Lambda}_{t}\left(P_{t}^{i}-\mathfrak{m}\left(\left(P_{t}\right)\right)\right)+\frac{\widehat{\beta}_{t}}{N}\right\} \mathrm{d} t \\
\mathrm{~d} P_{t}^{0}=-\partial_{x} \bar{f}_{0}^{(N)}\left(t, X_{t}^{0}, c_{t}^{0}\right) \mathrm{d} t+Q_{t}^{0,0} \mathrm{~d} W_{t}^{0}+\sum_{j=1}^{N} Q_{t}^{0, j} \mathrm{~d} W_{t}^{j} \\
\mathrm{~d} Y_{t}^{i}=-\partial_{x} \bar{f}_{i}\left(t, X_{t}^{i}, c_{t}^{0}, c_{t}^{i}\right) \mathrm{d} t+Z_{t}^{i, 0} \mathrm{~d} W_{t}^{0}+\sum_{j=1}^{N} Z_{t}^{i, j} \mathrm{~d} W_{t}^{j} \\
\mathrm{~d} P_{t}^{i}=c_{i}^{f}\left(t, c_{t}^{0}, c_{t}^{i}\right) R_{t}^{i} d t+Q_{t}^{i, 0} \mathrm{~d} W_{t}^{0}+\sum_{j=1}^{N} Q_{t}^{i, j} \mathrm{~d} W_{t}^{j}
\end{array}\right.
$$

with

$$
\left\{\begin{array}{l}
X_{0}^{0}=N \chi^{0}, X_{0}^{i}=\xi^{i}, R_{0}^{i}=0 \\
P_{T}^{0}=\partial_{x} g_{0}^{(N)}\left(X_{T}^{0}, c_{T}^{0}\right) \\
Y_{T}^{i}=\frac{\delta}{1-\delta} \mathfrak{m}\left(\left(c_{j}^{g}\left(c_{T}^{0}, c_{T}^{j}\right) X_{T}^{j}+h_{j}^{g}\left(c_{T}^{0}, c_{T}^{j}\right)\right)_{j=1}^{N}\right)+c_{i}^{g}\left(c_{T}^{0}, c_{T}^{i}\right) X_{T}^{i}+h_{i}^{g}\left(c_{T}^{0}, c_{T}^{i}\right) \\
P_{T}^{i}=-c_{i}^{g}\left(c_{T}^{0}, c_{T}^{i}\right)\left(R_{T}^{i}+\frac{\delta}{1-\delta} \mathfrak{m}\left(\left(R_{T}\right)\right)\right)
\end{array}\right.
$$

for $1 \leq i \leq N$. Here, $\left(\widehat{\beta}_{t}\right)_{t \in[0, T)}$ is defined by

$$
\widehat{\beta}_{t}=N \overline{\mathcal{V}}_{t}^{0}\left(-P_{t}^{0}+\mathfrak{m}\left(\left(Y_{t}\right)\right)+\mathfrak{m}\left(\left(P_{t}\right)\right)\right), t \in[0, T)
$$

The main result of this section is the next theorem.

Theorem 3.14. Under Assumptions (Minor-A, B) and (Major), there exists a unique strong solution $X^{0}, Y^{i}, P^{0}, P^{i}, R^{i} \in \mathbb{S}^{2}\left(\mathbb{F} ; \mathbb{R}^{n}\right), Z^{i, 0}, Q^{0,0}, Q^{i, 0} \in \mathbb{H}^{2}\left(\mathbb{F} ; \mathbb{R}^{n \times d_{0}}\right)$, and $Z^{i, j}, Q^{0, j}, Q^{i, j} \in \mathbb{H}^{2}\left(\mathbb{F} ; \mathbb{R}^{n \times d}\right), 1 \leq i, j \leq N$ to the coupled system of FBSDEs (3.17) with (3.18).

Proof. We shall show that the monotone conditions used in Theorem 2.6 in [49] are actually satisfied. Let $x^{0}, p^{0}$ and $x^{i}, y^{i}, p^{i}, r^{i}, 1 \leq i \leq N$ be arbitrary constants in $\mathbb{R}^{n}$. We put $x=\left(x^{i}\right)_{i=1}^{N}, y=\left(y^{i}\right)_{i=1}^{N}, p=\left(p^{i}\right)_{i=1}^{N}, r=$ $\left(r^{i}\right)_{i=1}^{N}$, and $u=\left(x^{0}, x, r, p^{0}, y, p\right)$. We write $\widehat{\beta}(t, u):=\overline{\mathcal{V}}_{t}^{0}\left(-p^{0}+\mathfrak{m}((y))+\mathfrak{m}((p))\right)$. As in Theorem 3.7, we 
introduce the quantities:

$$
\begin{aligned}
\operatorname{drift}\left[x^{0}\right](t, u) & :=\widehat{\beta}(t, u)+l_{0}^{(N)}\left(t, c_{t}^{0}\right), \\
\operatorname{drift}\left[x^{i}\right](t, u) & :=-\bar{\Lambda}_{t}\left(y^{i}-\mathfrak{m}((y))\right)-\frac{\widehat{\beta}(t, u)}{N}+l_{i}\left(t, c_{t}^{0}, c_{t}^{i}\right), \\
\operatorname{drift}\left[r^{i}\right](t, u) & :=\bar{\Lambda}_{t}\left(p^{i}-\mathfrak{m}((p))\right)+\frac{\widehat{\beta}(t, u)}{N}, \\
\operatorname{drift}\left[p^{0}\right](t, u) & :=-\partial_{x} \bar{f}_{0}^{(N)}\left(t, x^{0}, c_{t}^{0}\right), \\
\operatorname{drift}\left[y^{i}\right](t, u) & :=-\partial_{x} \bar{f}_{i}\left(t, x^{i}, c_{t}^{0}, c_{t}^{i}\right) \\
\operatorname{drift}\left[p^{i}\right](t, u) & :=c_{i}^{f}\left(t, c_{t}^{0}, c_{t}^{i}\right) r^{i},
\end{aligned}
$$

and

$$
\begin{aligned}
& \operatorname{terminal}\left[p^{0}\right](u):=\partial_{x} g_{0}^{(N)}\left(x^{0}, c_{T}^{0}\right), \\
& \operatorname{terminal}\left[y^{i}\right](u):=\frac{\delta}{1-\delta} \mathfrak{m}\left(\left(c_{j}^{g}\left(c_{T}^{0}, c_{T}^{j}\right) x^{j}+h_{j}^{g}\left(c_{T}^{0}, c_{T}^{j}\right)\right)_{j=1}^{N}\right)+c_{i}^{g}\left(c_{T}^{0}, c_{T}^{i}\right) x^{i}+h_{i}^{g}\left(c_{T}^{0}, c_{T}^{i}\right), \\
& \operatorname{terminal}\left[p^{i}\right](u):=-c_{i}^{g}\left(c_{T}^{0}, c_{T}^{i}\right)\left(r^{i}+\frac{\delta}{1-\delta} \mathfrak{m}((r))\right) .
\end{aligned}
$$

With two inputs $\left(u, u^{\prime}\right)$, we define $\Delta u:=u-u^{\prime}$,

$$
\begin{aligned}
& \Delta \operatorname{drift}\left[x^{0}\right](t):=\operatorname{drift}\left[x^{0}\right](t, u)-\operatorname{drift}\left[x^{0}\right]\left(t, u^{\prime}\right), \\
& \Delta \operatorname{terminal}\left[p^{0}\right]:=\operatorname{terminal}\left[p^{0}\right](u)-\operatorname{temrinal}\left[p^{0}\right]\left(u^{\prime}\right),
\end{aligned}
$$

and similarly for the others. From Remark 3.10, we have

$$
\begin{aligned}
\left\langle\Delta \operatorname{drift}\left[p^{0}\right](t), \Delta x^{0}\right\rangle & =-\left\langle\partial_{x} \overline{\mathfrak{f}}_{0}\left(t, x^{0} / N, c_{t}^{0}\right)-\partial_{x} \overline{\mathfrak{f}}_{0}\left(t, x^{0 \prime} / N, c_{t}^{0}\right), \Delta x_{t}^{0}\right\rangle \\
& \leq-N \gamma_{0}^{f}\left|\Delta x^{0} / N\right|^{2}=-\gamma_{0}^{f(N)}\left|\Delta x^{0}\right|^{2} .
\end{aligned}
$$

It is then straightforward to get

$$
\begin{aligned}
& \left\langle\Delta \operatorname{drift}\left[p^{0}\right](t), \Delta x^{0}\right\rangle+\sum_{i=1}^{N}\left\langle\Delta \operatorname{drift}\left[y^{i}\right](t), \Delta x^{i}\right\rangle+\sum_{i=1}^{N}\left\langle(-I) \Delta \operatorname{drift}\left[p^{i}\right](t), \Delta r^{i}\right\rangle \\
& \leq-\gamma_{0}^{f(N)}\left|\Delta x^{0}\right|^{2}-\gamma^{f} \sum_{i=1}^{N}\left(\left|\Delta x^{i}\right|^{2}+\left|\Delta r^{i}\right|^{2}\right),
\end{aligned}
$$

where $I=I_{n \times n}$ is the identity matrix. Next, with $\Delta \widehat{\beta}_{t}:=\widehat{\beta}(t, u)-\widehat{\beta}\left(t, u^{\prime}\right)$, we have

$$
\begin{aligned}
& \sum_{i=1}^{N}\left\langle\Delta \operatorname{drift}\left[x^{i}\right](t), \Delta y^{i}\right\rangle=\sum_{i=1}^{N}\left\langle-\bar{\Lambda}_{t}\left(\Delta y^{i}-\mathfrak{m}((\Delta y))\right)-\frac{\Delta \widehat{\beta}_{t}}{N}, \Delta y^{i}\right\rangle \\
& \quad=-\sum_{i=1}^{N}\left\langle\bar{\Lambda}_{t} \Delta y^{i}, \Delta y^{i}\right\rangle+N\left\langle\bar{\Lambda}_{t} \mathfrak{m}((\Delta y)), \mathfrak{m}((\Delta y))\right\rangle-N\left\langle\frac{\Delta \widehat{\beta}_{t}}{N}, \mathfrak{m}((\Delta y))\right\rangle \leq-N\left\langle\frac{\Delta \widehat{\beta}_{t}}{N}, \mathfrak{m}((\Delta y))\right\rangle .
\end{aligned}
$$


By similar calculation, we get

$$
\begin{aligned}
& \left\langle\Delta \operatorname{drift}\left[x^{0}\right](t), \Delta p^{0}\right\rangle+\sum_{i=1}^{N}\left\langle\Delta \operatorname{drift}\left[x^{i}\right](t), \Delta y^{i}\right\rangle+\sum_{i=1}^{N}\left\langle(-I) \Delta \operatorname{drift}\left[r^{i}\right](t), \Delta p^{i}\right\rangle \\
& \leq-N\left\langle\frac{\Delta \widehat{\beta}_{t}}{N},-\Delta p^{0}+\mathfrak{m}((\Delta y))+\mathfrak{m}((\Delta p))\right\rangle=-N\left\langle\frac{\Delta \widehat{\beta}_{t}}{N},\left(\Lambda_{t}^{0}+2 \Lambda_{t}\right) \frac{\Delta \widehat{\beta}_{t}}{N}\right\rangle \leq 0 .
\end{aligned}
$$

Therefore, from the drift contribution, we eventually have

$$
\begin{aligned}
& \left\langle\Delta \operatorname{drift}\left[p^{0}\right](t), \Delta x^{0}\right\rangle+\sum_{i=1}^{N}\left\langle\Delta \operatorname{drift}\left[y^{i}\right](t), \Delta x^{i}\right\rangle+\sum_{i=1}^{N}\left\langle(-I) \Delta \operatorname{drift}\left[p^{i}\right](t), \Delta r^{i}\right\rangle \\
& +\left\langle\Delta \operatorname{drift}\left[x^{0}\right](t), \Delta p^{0}\right\rangle+\sum_{i=1}^{N}\left\langle\Delta \operatorname{drift}\left[x^{i}\right](t), \Delta y^{i}\right\rangle+\sum_{i=1}^{N}\left\langle(-I) \Delta \operatorname{drift}\left[r^{i}\right](t), \Delta p^{i}\right\rangle \\
& \leq-\gamma_{0}^{f(N)}\left|\Delta x^{0}\right|^{2}-\gamma^{f} \sum_{i=1}^{N}\left(\left|\Delta x^{i}\right|^{2}+\left|\Delta r^{i}\right|^{2}\right) .
\end{aligned}
$$

For the terminal conditions, by the similar calculation done in (3.9), we obtain

$$
\begin{aligned}
& \left\langle\Delta \text { terminal }\left[p^{0}\right], \Delta x^{0}\right\rangle+\sum_{i=1}^{N}\left\langle\Delta \text { terminal }\left[y^{i}\right], \Delta x^{i}\right\rangle+\sum_{i=1}^{N}\left\langle(-I) \Delta \text { terminal }\left[p^{i}\right], \Delta r^{i}\right\rangle \\
& \geq \gamma_{0}^{g(N)}\left|\Delta x^{0}\right|^{2}+\left(\gamma^{g}-\mathfrak{a}\right) \sum_{i=1}^{N}\left(\left|\Delta x^{i}\right|^{2}+\left|\Delta r^{i}\right|^{2}\right) .
\end{aligned}
$$

Using (3.19) and (3.20), we can now apply Theorem 2.6 in [49] with

$$
A(t, u)=\left(\begin{array}{cccccc}
I_{n \times n} & 0 & 0 & 0 & 0 & 0 \\
0 & \left(I_{n \times n}\right)^{N} & 0 & 0 & 0 & 0 \\
0 & 0 & \left(-I_{n \times n}\right)^{N} & 0 & 0 & 0 \\
0 & 0 & 0 & I_{n \times n} & 0 & 0 \\
0 & 0 & 0 & 0 & \left(I_{n \times n}\right)^{N} & 0 \\
0 & 0 & 0 & 0 & 0 & \left(-I_{n \times n}\right)^{N}
\end{array}\right)\left(\begin{array}{c}
\operatorname{drift}\left[p^{0}\right] \\
\operatorname{drift}[y] \\
\operatorname{drift}[p] \\
\operatorname{drift}\left[x^{0}\right] \\
\operatorname{drift}[x] \\
\operatorname{drift}[r]
\end{array}\right)(t, u)
$$

and

$$
G=\left(\begin{array}{ccc}
I_{n \times n} & 0 & 0 \\
0 & \left(I_{n \times n}\right)^{N} & 0 \\
0 & 0 & \left(-I_{n \times n}\right)^{N}
\end{array}\right)
$$

In particular, we have $\beta_{1}:=\min \left(\gamma_{0}^{f(N)}, \gamma^{f}\right)>0, \mu_{1}:=\min \left(\gamma_{0}^{g(N)}, \gamma^{g}-\mathfrak{a}\right)>0$. Note that the coefficients of the Brownian motions ( $\sigma_{i}$ etc.) are irrelevant since they are uncontrolled and state-independent. In fact, one 
can repeat the proof for Theorem 3.3 in [28] in essentially the same way by simply replacing the analysis for $d\left\langle\Delta y_{t}, \Delta x_{t}\right\rangle$ with that for

$$
d\left\langle\left(\begin{array}{c}
\Delta p_{t}^{0} \\
\Delta y_{t} \\
\Delta p_{t}
\end{array}\right), G\left(\begin{array}{c}
\Delta x_{t}^{0} \\
\Delta x_{t} \\
\Delta r_{t}
\end{array}\right)\right\rangle
$$

using the above estimates.

Thanks to Theorem 3.14, we now find the market-clearing price process is given by

$$
\varpi_{t}=-\mathfrak{m}\left(\left(Y_{t}\right)\right)+\Lambda_{t} \overline{\mathcal{V}}_{t}^{0}\left(-P_{t}^{0}+\mathfrak{m}\left(\left(Y_{t}\right)\right)+\mathfrak{m}\left(\left(P_{t}\right)\right)\right), \quad t \in[0, T)
$$

using the solutions to the system of FBSDEs (3.17) with (3.18). Note that the system of equations is coupled among the agents $1 \leq i \leq N$ by the interactions through the empirical means such as $\mathfrak{m}\left(\left(Y_{t}\right)\right)$. As in the case for the standard mean field game theory for Nash equilibrium, we can obtain a simpler decoupled system described by the FBSDE of McKean-Vlasov type in the large- $N$ limit. This is the major topic to be treated in the remainder of the work. In the next section, we study the mean-field limit of the corresponding problem under the assumption that the minor agents are homogeneous.

Remark 3.15 (on Nash equilibrium). The equilibrium in our model is characterized by the relation $\sum_{i=1}^{N} \widehat{\alpha}_{t}^{i}+$ $\widehat{\beta}_{t}=0, \mathrm{~d} t \otimes d \mathbb{P}$-a.e. Notice that this market-clearing equilibrium is a different concept from the Nash equilibrium. Since Nash equilibrium is characterized by the optimality of the value function of each agent with respect to his/her strategy while keeping the other agents' strategies unchanged, it inevitably violates the market-clearing condition and hence is inapplicable to our case. In fact, because of this reason, the market-clearing equilibrium is quite popular in standard economic theories.

\section{MEAN-FIELD EQUILIBRIUM}

Let us work on the probability space with $N=1$ in Section 2 , i.e. $(\Omega, \mathcal{F}, \mathbb{P}, \mathbb{F})=\left(\Omega^{1}, \mathcal{F}^{1}, \mathbb{P}^{1}, \mathbb{F}^{1}\right)$. In the following, we use the notation:

$$
\mathbb{E}_{t}^{0}[\cdot]:=\mathbb{E}\left[\cdot \mid \overline{\mathcal{F}}_{t}^{0}\right]
$$

Let us first introduce the following assumptions.

Assumption 4.1. (MFG)

(i) $\left(l, \sigma^{0}, \sigma, \bar{f}, \bar{g}, c^{f}, c^{g}, h^{f}, h^{g}\right)$ satisfy the same conditions corresponding to those for $\left(l_{i}, \sigma_{i}^{0}, \sigma_{i}, \bar{f}_{i}, \bar{g}_{i}\right.$, $c_{i}^{f}, c_{i}^{g}, h_{i}^{f}, h_{i}^{g}$ ) in Assumption (Minor-A).

(ii) There exists some $\overline{\mathcal{F}}_{T}^{0}$-measurable $\mathcal{S}^{n}$-valued random variable $\mathfrak{c}$ such that

$$
\mathfrak{a}:=\frac{\delta}{1-\delta}\left\|\mathfrak{c}-c^{g}\left(c_{T}^{0}, c_{T}^{1}\right)\right\|_{\infty}<\gamma^{g}
$$

(iii) For the other variables and functions, we assume the same conditions as those in Assumptions (Minor-A) and (Major).

For the space of admissible strategies $\mathbb{A}_{\mathrm{mfg}}^{0}:=\mathbb{H}^{2}\left(\overline{\mathbb{F}}^{0} ; \mathbb{R}^{n}\right) \cap\left\{\beta_{T}=0\right\}$, we suppose that the major agent tries to solve

$$
\inf _{\boldsymbol{\beta} \in \mathbb{A}_{\mathrm{mfg}}^{0}} \mathcal{J}_{0}(\boldsymbol{\beta})
$$


where

$$
\mathcal{J}_{0}(\boldsymbol{\beta}):=\mathbb{E}\left[\int_{0}^{T} \mathfrak{f}_{0}\left(t, x_{t}^{0}, \beta_{t},-\mathbb{E}_{t}^{0}\left[y_{t}^{1}\right]+\Lambda_{t} \beta_{t}, \Lambda_{t}^{0}, c_{t}^{0}\right) \mathrm{d} t+\mathfrak{g}_{0}\left(x_{T}^{0}, c_{T}^{0}\right)\right]
$$

subject to the following dynamic constraints:

$$
\left\{\begin{array}{l}
\mathrm{d} x_{t}^{0}=\left(\beta_{t}+\mathfrak{l}_{0}\left(t, c_{t}^{0}\right)\right) \mathrm{d} t+\mathfrak{s}_{0}\left(t, c_{t}^{0}\right) \mathrm{d} W_{t}^{0}, \\
\mathrm{~d} x_{t}^{1}=\left\{-\bar{\Lambda}_{t}\left(y_{t}^{1}-\mathbb{E}_{t}^{0}\left[y_{t}^{1}\right]\right)-\beta_{t}+l\left(t, c_{t}^{0}, c_{t}^{1}\right)\right\} \mathrm{d} t+\sigma^{0}\left(t, c_{t}^{0}, c_{t}^{1}\right) \mathrm{d} W_{t}^{0}+\sigma\left(t, c_{t}^{0}, c_{t}^{1}\right) \mathrm{d} W_{t}^{1}, \\
\mathrm{~d} y_{t}^{1}=-\partial_{x} \bar{f}\left(t, x_{t}^{1}, c_{t}^{0}, c_{t}^{1}\right) \mathrm{d} t+z_{t}^{1,0} \mathrm{~d} W_{t}^{0}+z_{t}^{1,1} \mathrm{~d} W_{t}^{1},
\end{array}\right.
$$

with

$$
\left\{\begin{array}{l}
x_{0}^{0}=\chi^{0}, \quad x_{0}^{1}=\xi^{1}, \\
y_{T}^{1}=\frac{\delta}{1-\delta} \mathbb{E}_{T}^{0}\left[c^{g}\left(c_{T}^{0}, c_{T}^{1}\right) x_{T}^{1}+h^{g}\left(c_{T}^{0}, c_{T}^{1}\right)\right]+c^{g}\left(c_{T}^{0}, c_{T}^{1}\right) x_{T}^{1}+h^{g}\left(c_{T}^{0}, c_{T}^{1}\right) .
\end{array}\right.
$$

Here, the problem for the major agent is the optimization with respect to the controlled-FBSDE of conditional McKean-Vlasov type. One can naturally expect the above formulation of the problem in the mean-field limit from the McKean-Vlasov FBSDEs given in [27] and the expression in (3.6)

Remark 4.2. Notice that, the above problem is well posed in the sense that for a given $\boldsymbol{\beta} \in \mathbb{A}_{\mathrm{mfg}}^{0}$, there exists a unique strong solution to (4.2) and the corresponding cost $\mathcal{J}_{0}(\boldsymbol{\beta})$ is finite. In particular, the unique existence for $\left(x^{1}, y^{1}\right)$ can be proved by a simple modification of Theorem 4.2 in [27].

Implied from (3.13), we consider the Hamiltonian

$$
H:[0, T] \times\left(\mathbb{R}^{n}\right)^{9} \times \mathcal{S}^{n} \times \mathcal{S}_{+}^{n} \times\left(\mathbb{R}^{n}\right)^{2} \rightarrow \mathbb{R}
$$

by

$$
\begin{aligned}
& H\left(t, x^{0}, x^{1}, y^{1}, \bar{y}^{1}, p^{0}, p^{1}, \bar{p}^{1}, r^{1}, \beta, \Lambda^{0}, \Lambda, c^{0}, c^{1}\right) \\
& \quad:=\left\langle p^{0}, \beta+\mathfrak{l}_{0}\left(t, c^{0}\right)\right\rangle+\left\langle p^{1},-\bar{\Lambda}\left(y^{1}-\bar{y}^{1}\right)+l\left(t, c^{0}, c^{1}\right)\right\rangle+\left\langle\bar{p}^{1},-\beta\right\rangle \\
& \quad+\left\langle r^{1},-\partial_{x} \bar{f}\left(t, x^{1}, c^{0}, c^{1}\right)\right\rangle+\left\langle\beta,-\bar{y}^{1}+\Lambda \beta\right\rangle+\frac{1}{2}\left\langle\beta, \Lambda^{0} \beta\right\rangle+\overline{\mathfrak{f}}_{0}\left(t, x^{0}, c^{0}\right) .
\end{aligned}
$$

It is important to observe that the map

$$
\left(x^{0}, x^{1}, y^{1}, \bar{y}^{1}, \beta\right) \mapsto H\left(t, x^{0}, x^{1}, y^{1}, \bar{y}^{1}, p^{0}, p^{1}, \bar{p}^{1}, r^{1}, \beta, \Lambda^{0}, \Lambda, c^{0}, c^{1}\right)
$$

is jointly convex and strictly convex in $\beta$ (and $x^{0}$ ). It is easy to find

$$
\widehat{\beta}=\overline{\mathcal{V}}^{0}\left(-p^{0}+\bar{y}^{1}+\bar{p}^{1}\right),
$$

with $\overline{\mathcal{V}}^{0}:=\left(\Lambda^{0}+2 \Lambda\right)^{-1}$ gives the minimizer of $H$ with respect to $\beta$. 
The relevant set of adjoint equations can be inferred from Appendix A combined with Chapter 6 in [10], or from (3.17) and (3.18).

$$
\left\{\begin{array}{l}
\mathrm{d} r_{t}^{1}=\left\{\bar{\Lambda}_{t}\left(p_{t}^{1}-\mathbb{E}_{t}^{0}\left[p_{t}^{1}\right]\right)+\beta_{t}\right\} \mathrm{d} t \\
\mathrm{~d} p_{t}^{0}=-\partial_{x} \overline{\mathfrak{f}}_{0}\left(t, x_{t}^{0}, c_{t}^{0}\right) \mathrm{d} t+q_{t}^{0,0} \mathrm{~d} W_{t}^{0}, \\
\mathrm{~d} p_{t}^{1}=c^{f}\left(t, c_{t}^{0}, c_{t}^{1}\right) r_{t}^{1} \mathrm{~d} t+q_{t}^{1,0} \mathrm{~d} W_{t}^{0}+q_{t}^{1,1} \mathrm{~d} W_{t}^{1}
\end{array}\right.
$$

with

$$
\left\{\begin{array}{l}
r_{0}^{1}=0 \\
p_{T}^{0}=\partial_{x} \mathfrak{g}_{0}\left(x_{T}^{0}, c_{T}^{0}\right) \\
p_{T}^{1}=-c^{g}\left(c_{T}^{0}, c_{T}^{1}\right)\left(r_{T}^{1}+\frac{\delta}{1-\delta} \mathbb{E}_{T}^{0}\left[r_{T}^{1}\right]\right)
\end{array}\right.
$$

In order to guarantee that the above system characterizes the optimal solution for the major agent, we are going to prove the new verification theorem for controlled FBSDEs of conditional McKean-Vlasov type.

Theorem 4.3. Let Assumption $(M F G)$ be in force. Suppose that there exists a solution $\left(\widehat{x}^{0}, \widehat{x}^{1}, \widehat{r}^{1}, \widehat{p}^{0}, \widehat{p}^{1}, \widehat{y}^{1}\right)$ to $((4.2),(4.3))$ and $((4.5),(4.6))$ with the control process $\beta$ satisfying

$$
\widehat{\beta}_{t}=\overline{\mathcal{V}}_{t}^{0}\left(-\widehat{p}_{t}^{0}+\mathbb{E}_{t}^{0}\left[\widehat{y}_{t}^{1}\right]+\mathbb{E}_{t}^{0}\left[\hat{p}_{t}^{1}\right]\right)
$$

$\mathrm{d} t \otimes d \mathbb{P}$-a.e., then $\left(\widehat{\beta}_{t}\right)_{t \in[0, T)}$ (with $\left.\widehat{\beta}_{T}=0\right)$ is the unique optimal control for the problem (4.1).

Proof. For a given $\beta \in \mathbb{A}_{\mathrm{mfg}}^{0}$, we denote the associated solution to $(4.2)$ by $\left(x^{0}, x^{1}, y^{1}\right)$. We shall study the difference:

$$
\begin{aligned}
& \mathcal{J}_{0}(\boldsymbol{\beta})-\mathcal{J}_{0}(\widehat{\boldsymbol{\beta}})=\mathbb{E}\left[\mathfrak{g}_{0}\left(x_{T}^{0}, c_{T}^{0}\right)-\mathfrak{g}_{0}\left(\widehat{x}_{T}^{0}, c_{T}^{0}\right)\right. \\
& \left.\quad+\int_{0}^{T}\left(\mathfrak{f}_{0}\left(t, x_{t}^{0}, \beta_{t},-\mathbb{E}_{t}^{0}\left[y_{t}^{1}\right]+\Lambda_{t} \beta_{t}, \Lambda_{t}^{0}, c_{t}^{0}\right)-\mathfrak{f}_{0}\left(t, \widehat{x}_{t}^{0}, \widehat{\beta}_{t},-\mathbb{E}_{t}^{0}\left[\widehat{y}_{t}^{1}\right]+\Lambda_{t} \widehat{\beta}_{t}, \Lambda_{t}^{0}, c_{t}^{0}\right)\right) \mathrm{d} t\right] .
\end{aligned}
$$

First, observe that

$$
\begin{aligned}
\mathbb{E} & {\left[\left\langle\widehat{p}_{T}^{1}, x_{T}^{1}-\widehat{x}_{T}^{1}\right\rangle+\left\langle\widehat{r}_{T}^{1}, y_{T}^{1}-\widehat{y}_{T}^{1}\right\rangle\right] } \\
& =\frac{\delta}{1-\delta} \mathbb{E}\left[-\left\langle\mathbb{E}_{T}^{0}\left[\widehat{r}_{T}^{1}\right], c^{g}\left(c_{T}^{0}, c_{T}^{1}\right)\left(x_{T}^{1}-\widehat{x}_{T}^{1}\right)\right\rangle+\left\langle\widehat{r}_{T}^{1}, \mathbb{E}_{T}^{0}\left[c^{g}\left(c_{T}^{0}, c_{T}^{1}\right)\left(x_{T}^{1}-\widehat{x}_{T}^{1}\right)\right]\right\rangle\right] \\
& =0 .
\end{aligned}
$$

Thus, from the convexity of $\mathfrak{g}_{0}$, we have

$$
\mathbb{E}\left[\mathfrak{g}_{0}\left(x_{T}^{0}, c_{T}^{0}\right)-\mathfrak{g}_{0}\left(\widehat{x}_{T}^{0}, c_{T}^{0}\right)\right] \geq \mathbb{E}\left[\left\langle\widehat{p}_{T}^{0}, x_{T}^{0}-\widehat{x}_{T}^{0}\right\rangle+\left\langle\widehat{p}_{T}^{1}, x_{T}^{1}-\widehat{x}_{T}^{1}\right\rangle+\left\langle\widehat{r}_{T}^{1}, y_{T}^{1}-\widehat{y}_{T}^{1}\right\rangle\right]
$$


Let us use $\widehat{\Theta}_{t}:=\left(\widehat{x}_{t}^{0}, \widehat{x}_{t}^{1}, \widehat{y}_{t}^{1}, \mathbb{E}_{t}^{0}\left[\widehat{y}_{t}^{1}\right], \widehat{p}_{t}^{0}, \widehat{p}_{t}^{1}, \mathbb{E}_{t}^{0}\left[\widehat{p}_{t}^{1}\right], \widehat{r}_{t}^{1}\right), \widehat{\theta}_{t}:=\left(\widehat{p}_{t}^{0}, \widehat{p}_{t}^{1}, \mathbb{E}_{t}^{0}\left[\widehat{p}_{t}^{1}\right], \widehat{r}_{t}^{1}\right)$ and omit the common arguments $\left(\Lambda_{t}^{0}, \Lambda_{t}, c_{t}^{0}, c_{t}^{1}\right)$ in the Hamiltonian. Since $\bar{\Lambda}, \widehat{\beta}$ are $\overline{\mathbb{F}}^{0}$-adapted, we have

$$
\begin{aligned}
\mathbb{E} & {\left[\left\langle\bar{\Lambda}_{t}\left(\widehat{p}_{t}^{1}-\mathbb{E}_{t}^{0}\left[\widehat{p}_{t}^{1}\right]\right)+\widehat{\beta}_{t}, y_{t}^{1}-\widehat{y}_{t}^{1}\right\rangle\right] } \\
& =\mathbb{E}\left[\left\langle\bar{\Lambda}_{t} \widehat{p}_{t}^{1}, y_{t}^{1}-\widehat{y}_{t}^{1}\right\rangle\right]+\mathbb{E}\left[\left\langle-\bar{\Lambda}_{t} \mathbb{E}_{t}^{0}\left[\widehat{p}_{t}^{1}\right]+\widehat{\beta}_{t}, y_{t}^{1}-\widehat{y}_{t}^{1}\right\rangle\right] \\
& =\mathbb{E}\left[\left\langle\bar{\Lambda}_{t} \widehat{p}_{t}^{1}, y_{t}^{1}-\widehat{y}_{t}^{1}\right\rangle\right]+\mathbb{E}\left[\left\langle-\bar{\Lambda}_{t} \widehat{p}_{t}^{1}+\widehat{\beta}_{t}, \mathbb{E}_{t}^{0}\left[y_{t}^{1}\right]-\mathbb{E}_{t}^{0}\left[\widehat{y}_{t}^{1}\right]\right\rangle\right] \\
& =\mathbb{E}\left[-\left\langle\partial_{y^{1}} H\left(t, \widehat{\Theta}_{t}, \widehat{\beta}_{t}\right), y_{t}^{1}-\widehat{y}_{t}^{1}\right\rangle-\left\langle\partial_{\bar{y}^{1}} H\left(t, \widehat{\Theta}_{t}, \widehat{\beta}_{t}\right), \mathbb{E}_{t}^{0}\left[y_{t}^{1}\right]-\mathbb{E}_{t}^{0}\left[\widehat{y}_{t}^{1}\right]\right\rangle\right] .
\end{aligned}
$$

With these results and (4.7), a simple application of Itô-formula yields

$$
\begin{aligned}
\mathcal{J}_{0}(\boldsymbol{\beta})- & \mathcal{J}_{0}(\widehat{\boldsymbol{\beta}}) \\
\geq \mathbb{E} \int_{0}^{T} & {\left[H\left(t, x_{t}^{0}, x_{t}^{1}, y_{t}^{1}, \mathbb{E}_{t}^{0}\left[y_{t}^{1}\right], \widehat{\theta}_{t}, \beta_{t}\right)-H\left(t, \widehat{\Theta}_{t}, \widehat{\beta}_{t}\right)\right.} \\
& -\left\langle\partial_{x^{0}} H\left(t, \widehat{\Theta}_{t}, \widehat{\beta}_{t}\right), x_{t}^{0}-\widehat{x}_{t}^{0}\right\rangle-\left\langle\partial_{x^{1}} H\left(t, \widehat{\Theta}_{t}, \widehat{\beta}_{t}\right), x_{t}^{1}-\widehat{x}_{t}^{1}\right\rangle \\
& \left.-\left\langle\partial_{y^{1}} H\left(t, \widehat{\Theta}_{t}, \widehat{\beta}_{t}\right), y_{t}^{1}-\widehat{y}_{t}^{1}\right\rangle-\left\langle\partial_{\bar{y}^{1}} H\left(t, \widehat{\Theta}_{t}, \widehat{\beta}_{t}\right), \mathbb{E}_{t}^{0}\left[y_{t}^{1}\right]-\mathbb{E}_{t}^{0}\left[\widehat{y}_{t}^{1}\right]\right\rangle\right] \mathrm{d} t \\
\geq \mathbb{E} \int_{0}^{T} & {\left[H\left(t, x_{t}^{0}, x_{t}^{1}, y_{t}^{1}, \mathbb{E}_{t}^{0}\left[y_{t}^{1}\right], \widehat{\theta}_{t}, \beta_{t}\right)-H\left(t, \widehat{\Theta}_{t}, \widehat{\beta}_{t}\right)\right.} \\
& \quad-\left\langle\partial_{x^{0}} H\left(t, \widehat{\Theta}_{t}, \widehat{\beta}_{t}\right), x_{t}^{0}-\widehat{x}_{t}^{0}\right\rangle-\left\langle\partial_{x^{1}} H\left(t, \widehat{\Theta}_{t}, \widehat{\beta}_{t}\right), x_{t}^{1}-\widehat{x}_{t}^{1}\right\rangle \\
& \quad-\left\langle\partial_{y^{1}} H\left(t, \widehat{\Theta}_{t}, \widehat{\beta}_{t}\right), y_{t}^{1}-\widehat{y}_{t}^{1}\right\rangle-\left\langle\partial_{\bar{y}^{1}} H\left(t, \widehat{\Theta}_{t}, \widehat{\beta}_{t}\right), \mathbb{E}_{t}^{0}\left[y_{t}^{1}\right]-\mathbb{E}_{t}^{0}\left[\widehat{y}_{t}^{1}\right]\right\rangle \\
& \left.\quad-\left\langle\partial_{\beta} H\left(t, \widehat{\Theta}_{t}, \widehat{\beta}_{t}\right), \beta_{t}-\widehat{\beta}_{t}\right\rangle\right] \mathrm{d} t \\
\geq 0, &
\end{aligned}
$$

where the second inequality follows from the fact that $\widehat{\beta}_{t}=\operatorname{argmin}_{\beta} H\left(t, \widehat{\Theta}_{t}, \beta\right)$. The equality holds only when $\beta=\widehat{\beta}$ due to the strict convexity.

From Theorem 4.3, it is clear that the relevant set of equations is given by

$$
\left\{\begin{array}{l}
\mathrm{d} x_{t}^{0}=\left(\widehat{\beta}_{t}+\mathfrak{l}_{0}\left(t, c_{t}^{0}\right)\right) \mathrm{d} t+\mathfrak{s}_{0}\left(t, c_{t}^{0}\right) \mathrm{d} W_{t}^{0}, \\
\mathrm{~d} x_{t}^{1}=\left\{-\bar{\Lambda}_{t}\left(y_{t}^{1}-\mathbb{E}_{t}^{0}\left[y_{t}^{1}\right]\right)-\widehat{\beta}_{t}+l\left(t, c_{t}^{0}, c_{t}^{1}\right)\right\} \mathrm{d} t+\sigma^{0}\left(t, c_{t}^{0}, c_{t}^{1}\right) \mathrm{d} W_{t}^{0}+\sigma\left(t, c_{t}^{0}, c_{t}^{1}\right) \mathrm{d} W_{t}^{1}, \\
\mathrm{~d} r_{t}^{1}=\left\{\bar{\Lambda}_{t}\left(p_{t}^{1}-\mathbb{E}_{t}^{0}\left[p_{t}^{1}\right]\right)+\widehat{\beta}_{t}\right\} \mathrm{d} t \\
\mathrm{~d} p_{t}^{0}=-\partial_{x} \overline{\mathfrak{f}}_{0}\left(t, x_{t}^{0}, c_{t}^{0}\right) \mathrm{d} t+q_{t}^{0,0} \mathrm{~d} W_{t}^{0}, \\
\mathrm{~d} y_{t}^{1}=-\partial_{x} \bar{f}\left(t, x_{t}^{1}, c_{t}^{0}, c_{t}^{1}\right) \mathrm{d} t+z_{t}^{1,0} \mathrm{~d} W_{t}^{0}+z_{t}^{1,1} \mathrm{~d} W_{t}^{1}, \\
\mathrm{~d} p_{t}^{1}=c^{f}\left(t, c_{t}^{0}, c_{t}^{1}\right) r_{t}^{1} d t+q_{t}^{1,0} \mathrm{~d} W_{t}^{0}+q_{t}^{1,1} \mathrm{~d} W_{t}^{1},
\end{array}\right.
$$

with

$$
\left\{\begin{array}{l}
x_{0}^{0}=\chi^{0}, \quad x_{0}^{1}=\xi^{1}, \quad r_{0}^{1}=0 \\
p_{T}^{0}=\partial_{x} \mathfrak{g}_{0}\left(x_{T}^{0}, c_{T}^{0}\right), \\
y_{T}^{1}=\frac{\delta}{1-\delta} \mathbb{E}_{T}^{0}\left[c^{g}\left(c_{T}^{0}, c_{T}^{1}\right) x_{T}^{1}+h^{g}\left(c_{T}^{0}, c_{T}^{1}\right)\right]+c^{g}\left(c_{T}^{0}, c_{T}^{1}\right) x_{T}^{1}+h^{g}\left(c_{T}^{0}, c_{T}^{1}\right), \\
p_{T}^{1}=-c^{g}\left(c_{T}^{0}, c_{T}^{1}\right)\left(r_{T}^{1}+\frac{\delta}{1-\delta} \mathbb{E}_{T}^{0}\left[r_{T}^{1}\right]\right),
\end{array}\right.
$$


where $\widehat{\beta}_{t}, t \in[0, T)$ is defined by

$$
\widehat{\beta}_{t}:=\overline{\mathcal{V}}_{t}^{0}\left(-p_{t}^{0}+\mathbb{E}_{t}^{0}\left[y_{t}^{1}\right]+\mathbb{E}_{t}^{0}\left[p_{t}^{1}\right]\right)
$$

The next theorem guarantees the existence of the solution to the above FBSDE and hence the optimal control for the major agent in the mean-field limit.

Theorem 4.4. Under the Assumption $(M F G)$, there exists a unique strong solution $x^{0}, p^{0} \in \mathbb{S}^{2}\left(\overline{\mathbb{F}}^{0} ; \mathbb{R}^{n}\right)$, $x^{1}, r^{1}, y^{1}, p^{1} \in \mathbb{S}^{2}\left(\mathbb{F}^{1} ; \mathbb{R}^{n}\right), q^{0,0} \in \mathbb{H}^{2}\left(\overline{\mathbb{F}}^{0} ; \mathbb{R}^{n \times d_{0}}\right), z^{1,0}, q^{1,0} \in \mathbb{H}^{2}\left(\mathbb{F}^{1} ; \mathbb{R}^{n \times d_{0}}\right)$ and $z^{1,1}, q^{1,1} \in \mathbb{H}^{2}\left(\mathbb{F}^{1} ; \mathbb{R}^{n \times d}\right)$ to the system of FBSDEs of conditional McKean-Vlasov type (4.8) with (4.9).

Proof. As we have done in the proof for Theorem 3.14, we introduce $u:=\left(x^{0}, x^{1}, r^{1}, p^{0}, y^{1}, p^{1}\right)$ as arbitrary square integrable random variables with appropriate dimensions satisfying that $\left(x^{0}, p^{0}\right)$ are $\overline{\mathcal{F}}_{t}^{0}$-measurable, and the others are $\mathcal{F}_{t}^{1}$-measurable. For these inputs, we define

$$
\begin{aligned}
\operatorname{drift}\left[x^{0}\right](t, u) & :=\widehat{\beta}(t, u)+\mathfrak{l}_{0}\left(t, c_{t}^{0}\right), \\
\operatorname{drift}\left[x^{1}\right](t, u) & :=-\bar{\Lambda}_{t}\left(y^{1}-\mathbb{E}_{t}^{0}\left[y^{1}\right]\right)-\widehat{\beta}(t, u)+l\left(t, c_{t}^{0}, c_{t}^{1}\right), \\
\operatorname{drift}\left[r^{1}\right](t, u) & :=\bar{\Lambda}_{t}\left(p^{1}-\mathbb{E}_{t}^{0}\left[p^{1}\right]\right)+\widehat{\beta}(t, u), \\
\operatorname{drift}\left[p^{0}\right](t, u) & :=-\partial_{x} \overline{\mathfrak{f}}_{0}\left(t, x^{0}, c_{t}^{0}\right), \\
\operatorname{drift}\left[y^{1}\right](t, u) & :=-\partial_{x} \bar{f}\left(t, x^{1}, c_{t}^{0}, c_{t}^{1}\right), \\
\operatorname{drift}\left[p^{1}\right](t, u) & :=c^{f}\left(t, c_{t}^{0}, c_{t}^{1}\right) r^{1},
\end{aligned}
$$

where $\widehat{\beta}(t, u):=\overline{\mathcal{V}}_{t}^{0}\left(-p^{0}+\mathbb{E}_{t}^{0}\left[y^{1}\right]+\mathbb{E}_{t}^{0}\left[p^{1}\right]\right)$. For two different inputs $u, u^{\prime}$, we set $\Delta u:=u-u^{\prime}, \Delta \operatorname{drift}\left[x^{0}\right](t):=$ $\operatorname{drift}\left[x^{0}\right](t, u)-\operatorname{drift}\left[x^{0}\right]\left(t, u^{\prime}\right)$ and similarly for the other quantities, too. Since $\overline{\mathcal{V}}_{t}^{0}$ and $p^{0}$ are $\overline{\mathcal{F}}_{t}^{0}$-measurable, we see

$$
\begin{aligned}
\mathbb{E}_{t}^{0} & {\left[\left\langle\Delta \operatorname{drift}\left[x^{0}\right](t), \Delta p^{0}\right\rangle+\left\langle\Delta \operatorname{drift}\left[x^{1}\right](t), \Delta y^{1}\right\rangle+\left\langle(-I) \Delta \operatorname{drift}\left[r^{1}\right](t), \Delta p^{1}\right\rangle\right] } \\
& =-\mathbb{E}_{t}^{0}\left[\left\langle\overline{\mathcal{V}}_{t}^{0}\left(-\Delta p^{0}+\mathbb{E}_{t}^{0}\left[\Delta y^{1}+\Delta p^{1}\right]\right),-\Delta p^{0}+\Delta y^{1}+\Delta p^{1}\right\rangle\right] \\
& =-\left\langle\overline{\mathcal{V}}_{t}^{0}\left(-\Delta p^{0}+\mathbb{E}_{t}^{0}\left[\Delta y^{1}+\Delta p^{1}\right]\right),-\Delta p^{0}+\mathbb{E}_{t}^{0}\left[\Delta y^{1}+\Delta p^{1}\right]\right\rangle \leq 0 .
\end{aligned}
$$

It is straightforward to check

$$
\begin{aligned}
& \mathbb{E}\left[\left\langle\Delta \operatorname{drift}\left[p^{0}\right](t), \Delta x^{0}\right\rangle+\left\langle\Delta \operatorname{drift}\left[y^{1}\right](t), \Delta x^{1}\right\rangle+\left\langle(-I) \Delta \operatorname{drift}\left[p^{1}\right](t), \Delta r^{1}\right\rangle\right] \\
& \leq-\gamma_{0}^{f} \mathbb{E}\left|\Delta x^{0}\right|^{2}-\gamma^{f}\left(\mathbb{E}\left|\Delta x^{1}\right|^{2}+\mathbb{E}\left|\Delta r^{1}\right|^{2}\right) .
\end{aligned}
$$

Now we set $v:=\left(x^{0}, x^{1}, r^{1}\right)$ as arbitrary square integrable random variables with appropriate dimensions satisfying that $x^{0}$ are $\overline{\mathcal{F}}_{T}^{0}$-measurable, and the others are $\mathcal{F}_{T}^{1}$-measurable. For these inputs, let us define

$$
\begin{aligned}
\operatorname{terminal}\left[p^{0}\right](v) & :=\partial_{x} \mathfrak{g}_{0}\left(x^{0}, c_{T}^{0}\right), \\
\operatorname{terminal}\left[y^{1}\right](v) & :=\frac{\delta}{1-\delta} \mathbb{E}_{T}^{0}\left[c^{g}\left(c_{T}^{0}, c_{T}^{1}\right) x^{1}+h^{g}\left(c_{T}^{0}, c_{T}^{1}\right)\right]+c^{g}\left(c_{T}^{0}, c_{T}^{1}\right) x^{1}+h^{g}\left(c_{T}^{0}, c_{T}^{1}\right), \\
\operatorname{terminal}\left[p^{1}\right](v) & :=-c^{g}\left(c_{T}^{0}, c_{T}^{1}\right)\left(r^{1}+\frac{\delta}{1-\delta} \mathbb{E}_{T}^{0}\left[r^{1}\right]\right),
\end{aligned}
$$


and with two different input $v, v^{\prime}$, we denote by $\Delta v:=v-v^{\prime}$,

$$
\Delta \text { terminal }\left[p^{0}\right]:=\operatorname{terminal}\left[p^{0}\right](v)-\operatorname{terminal}\left[p^{0}\right]\left(v^{\prime}\right)
$$

and similarly for the other quantities. Observe that

$$
\begin{aligned}
\mathbb{E}_{T}^{0} & {\left[\left\langle\Delta \text { terminal }\left[y^{1}\right], \Delta x^{1}\right\rangle\right] } \\
& =\mathbb{E}_{T}^{0}\left[\left\langle\frac{\delta}{1-\delta} \mathbb{E}_{T}^{0}\left[c^{g}\left(c_{T}^{0}, c_{T}^{1}\right) \Delta x^{1}\right]+c^{g}\left(c_{T}^{0}, c_{T}^{1}\right) \Delta x^{1}, \Delta x^{1}\right\rangle\right] \\
& \geq \gamma^{g} \mathbb{E}_{T}^{0}\left|\Delta x^{1}\right|^{2}+\frac{\delta}{1-\delta}\left\langle\mathfrak{c} \mathbb{E}_{T}^{0}\left[\Delta x^{1}\right], \mathbb{E}_{T}^{0}\left[\Delta x^{1}\right]\right\rangle+\frac{\delta}{1-\delta}\left\langle\mathbb{E}_{T}^{0}\left[\left(c^{g}\left(c_{T}^{0}, c_{T}^{1}\right)-\mathfrak{c}\right) \Delta x^{1}\right], \mathbb{E}_{T}^{0}\left[\Delta x^{1}\right]\right\rangle \\
& \geq\left(\gamma^{g}-\mathfrak{a}\right) \mathbb{E}_{T}^{0}\left|\Delta x^{1}\right|^{2} .
\end{aligned}
$$

Similar calculation yields

$$
\begin{aligned}
& \mathbb{E}\left[\left\langle\Delta \text { terminal }\left[p^{0}\right], \Delta x^{0}\right\rangle+\left\langle\Delta \operatorname{terminal}\left[y^{1}\right], \Delta x^{1}\right\rangle+\left\langle(-I) \Delta \text { terminal }\left[p^{1}\right], \Delta r^{1}\right\rangle\right] \\
& \quad \geq \gamma_{0}^{g} \mathbb{E}\left|\Delta x^{0}\right|^{2}+\left(\gamma^{g}-\mathfrak{a}\right) \mathbb{E}\left[\left|\Delta x^{1}\right|^{2}+\left|\Delta r^{1}\right|^{2}\right] .
\end{aligned}
$$

We have now obtained the monotone conditions necessary for the method of continuation. In particular, by introducing a strictly positive constant $\gamma>0$

$$
\gamma:=\min \left\{\gamma_{0}^{f}, \gamma^{f}, \gamma_{0}^{g}, \gamma^{g}-\mathfrak{a}\right\},
$$

we have from (4.10), (4.11) and (4.12)

$$
\begin{aligned}
& \mathbb{E}\left[\left\langle\Delta \operatorname{drift}\left[x^{0}\right](t), \Delta p^{0}\right\rangle+\left\langle\Delta \operatorname{drift}\left[x^{1}\right](t), \Delta y^{1}\right\rangle+\left\langle(-I) \Delta \operatorname{drift}\left[r^{1}\right](t), \Delta p^{1}\right\rangle\right] \leq 0, \\
& \mathbb{E}\left[\left\langle\Delta \operatorname{drift}\left[p^{0}\right](t), \Delta x^{0}\right\rangle+\left\langle\Delta \operatorname{drift}\left[y^{1}\right](t), \Delta x^{1}\right\rangle+\left\langle(-I) \Delta \operatorname{drift}\left[p^{1}\right](t), \Delta r^{1}\right\rangle\right] \\
& \quad \leq-\gamma \mathbb{E}\left[\left|\Delta x^{0}\right|^{2}+\left|\Delta x^{1}\right|^{2}+\left|\Delta r^{1}\right|^{2}\right], \\
& \mathbb{E}\left[\left\langle\Delta \operatorname{terminal}\left[p^{0}\right], \Delta x^{0}\right\rangle+\left\langle\Delta \operatorname{terminal}\left[y^{1}\right], \Delta x^{1}\right\rangle+\left\langle(-I) \Delta \operatorname{terminal}\left[p^{1}\right], \Delta r^{1}\right\rangle\right] \\
& \quad \geq \gamma \mathbb{E}\left[\left|\Delta x^{0}\right|^{2}+\left|\Delta x^{1}\right|^{2}+\left|\Delta r^{1}\right|^{2}\right] .
\end{aligned}
$$

We can now repeat the proof of Theorem 4.2 in [27]. The three conditions of (4.13) correspond to those of (4.3) (with $\left.L_{\varpi}=0\right)$ in [27]. We treat $\left(x_{t}^{0}, x_{t}^{1}, r_{t}^{1}\right)$ and $\left(p_{t}^{0}, y_{t}^{1}, p_{t}^{1}\right)$ as the tuple of forward and backward processes, which are represented by $X_{t}$ and $Y_{t}$ in [27], respectively. By replacing the estimate on $\mathbb{E}\left[\left\langle\Delta Y_{T}, \Delta X_{T}\right\rangle\right]$ by

$$
\mathbb{E}\left[\left\langle\left(\begin{array}{c}
\Delta p_{T}^{0} \\
\Delta y_{T}^{1} \\
\Delta p_{T}^{1}
\end{array}\right), G\left(\begin{array}{c}
\Delta x_{T}^{0} \\
\Delta x_{T}^{1} \\
\Delta r_{T}^{1}
\end{array}\right)\right\rangle\right]
$$

with $G=\left(\begin{array}{ccc}I_{n \times n} & 0 & 0 \\ 0 & \left(I_{n \times n}\right) & 0 \\ 0 & 0 & \left(-I_{n \times n}\right)\end{array}\right)$, we can follow the same procedures with slightly more cumbersome indexing.

Remark 4.5 (on Lasry-Lions monotonicity). The so-called Lasry-Lions monotonicity is a famous criterion for the uniqueness of the mean field games. It dates back to their original papers [41-43] and is defined as follows [10, Def. 3.28]: a real-valued function $h$ on $\mathbb{R}^{d} \times \mathcal{P}_{2}\left(\mathbb{R}^{d}\right)$ is said to be monotone in the sense of Lasry and Lions, if, 
for all $\mu \in \mathcal{P}_{2}\left(\mathbb{R}^{d}\right)$, the mapping $\mathbb{R}^{d} \ni x \mapsto h(x, \mu)$ is at most quadratic growth, and for all $\mu, \mu^{\prime} \in \mathcal{P}_{2}\left(\mathbb{R}^{d}\right)$, we have

$$
\int_{\mathbb{R}^{d}}\left(h(x, \mu)-h\left(x, \mu^{\prime}\right)\right) d\left(\mu-\mu^{\prime}\right)(x) \geq 0
$$

The uniqueness result in probabilistic settings is given by Theorem 3.29 in [10]. It says that there is at most one MFG equilibrium if the running as well as terminal cost functions satisfy Lasry-Lions monotonicity.

On the other hand, the relevant monotonicity in the current paper is assumed so that the continuation method [49] is applicable. See, for example, the set of inequalities (4.13). It essentially corresponds to [49, (H2.3)] and is used to make Banach's fixed point theorem applicable to prove the existence as well as the uniqueness of the solution.

\section{Convergence to the MeAn-Field Limit}

From Theorem 4.4, the market-clearing price in the mean-field limit is naturally expected to be

$$
\varpi_{t}^{\mathrm{mfg}}:=-\mathbb{E}_{t}^{0}\left[y_{t}^{1}\right]+\Lambda_{t} \overline{\mathcal{V}}_{t}^{0}\left(-p_{t}^{0}+\mathbb{E}_{t}^{0}\left[y_{t}^{1}\right]+\mathbb{E}_{t}^{0}\left[p_{t}^{1}\right]\right), \quad t \in[0, T) .
$$

In this section, we shall show that this is indeed the case for the homogeneous minor agents. Lastly, we also provide the estimate on the difference of the equilibrium price between the two markets; one is the homogeneous mean-field limit and the other is the heterogeneous market of finite population.

\subsection{Large population limit of the minor agents}

We now go back to the original setup of probability space given in Section 2. We first assume that the minor agents are homogeneous.

Assumption 5.1. (Minor-Homogeneous) The conditions in Assumption (MFG) hold true. Moreover, every minor agent $1 \leq i \leq N$ is subject to the common coefficient functions $\left(l, \sigma^{0}, \sigma, \bar{f}, \bar{g}, c^{f}, c^{g}, h^{f}, h^{g}\right)$ given there.

For each $1 \leq i \leq N$, let us construct $\mathbb{F}^{i}$-adapted processes, corresponding to those given by (4.8) and (4.9):

$$
\left\{\begin{aligned}
\mathrm{d} x_{t}^{0} & =\left\{\overline{\mathcal{V}}_{t}^{0}\left(-p_{t}^{0}+\mathbb{E}_{t}^{0}\left[y_{t}^{i}\right]+\mathbb{E}_{t}^{0}\left[p_{t}^{i}\right]\right)+\mathfrak{l}_{0}\left(t, c_{t}^{0}\right)\right\} \mathrm{d} t+\mathfrak{s}_{0}\left(t, c_{t}^{0}\right) \mathrm{d} W_{t}^{0}, \\
\mathrm{~d} x_{t}^{i}= & \left\{-\bar{\Lambda}_{t}\left(y_{t}^{i}-\mathbb{E}_{t}^{0}\left[y_{t}^{i}\right]\right)-\overline{\mathcal{V}}_{t}^{0}\left(-p_{t}^{0}+\mathbb{E}_{t}^{0}\left[y_{t}^{i}\right]+\mathbb{E}^{0}\left[p_{t}^{i}\right]\right)+l\left(t, c_{t}^{0}, c_{t}^{i}\right)\right\} \mathrm{d} t \\
& +\sigma^{0}\left(t, c_{t}^{0}, c_{t}^{i}\right) \mathrm{d} W_{t}^{0}+\sigma\left(t, c_{t}^{0}, c_{t}^{i}\right) \mathrm{d} W_{t}^{i}, \\
\mathrm{~d} r_{t}^{i}= & \left\{\bar{\Lambda}_{t}\left(p_{t}^{i}-\mathbb{E}_{t}^{0}\left[p_{t}^{i}\right]\right)+\overline{\mathcal{V}}_{t}^{0}\left(-p_{t}^{0}+\mathbb{E}_{t}^{0}\left[y_{t}^{i}\right]+\mathbb{E}_{t}^{0}\left[p_{t}^{i}\right]\right)\right\} \mathrm{d} t, \\
\mathrm{~d} p_{t}^{0}= & -\partial_{x} \overline{\mathfrak{f}}_{0}\left(t, x_{t}^{0}, c_{t}^{0}\right) \mathrm{d} t+q_{t}^{0,0} \mathrm{~d} W_{t}^{0}, \\
\mathrm{~d} y_{t}^{i}= & -\partial_{x} \bar{f}\left(t, x_{t}^{i}, c_{t}^{0}, c_{t}^{i}\right) \mathrm{d} t+z_{t}^{i, 0} \mathrm{~d} W_{t}^{0}+z_{t}^{i, i} \mathrm{~d} W_{t}^{i}, \\
\mathrm{~d} p_{t}^{i}= & c^{f}\left(t, c_{t}^{0}, c_{t}^{i}\right) r_{t}^{i} d t+q_{t}^{i, 0} \mathrm{~d} W_{t}^{0}+q_{t}^{i, i} \mathrm{~d} W_{t}^{i},
\end{aligned}\right.
$$

with

$$
\left\{\begin{array}{l}
x_{0}^{0}=\chi^{0}, \quad x_{0}^{i}=\xi^{i}, \quad r_{0}^{i}=0 \\
p_{T}^{0}=\partial_{x} \mathfrak{g}_{0}\left(x_{T}^{0}, c_{T}^{0}\right), \\
y_{T}^{i}=\frac{\delta}{1-\delta} \mathbb{E}_{T}^{0}\left[c^{g}\left(c_{T}^{0}, c_{T}^{i}\right) x_{T}^{i}+h^{g}\left(c_{T}^{0}, c_{T}^{i}\right)\right]+c^{g}\left(c_{T}^{0}, c_{T}^{i}\right) x_{T}^{i}+h^{g}\left(c_{T}^{0}, c_{T}^{i}\right), \\
p_{T}^{i}=-c^{g}\left(c_{T}^{0}, c_{T}^{i}\right)\left(r_{T}^{i}+\frac{\delta}{1-\delta} \mathbb{E}_{T}^{0}\left[r_{T}^{i}\right]\right) .
\end{array}\right.
$$


By construction of the probability space and the fact that $\left(c^{i}, \xi^{i}, W^{i}\right)$ are independently and identically distributed (i.i.d.), $\mathbb{F}^{i}$-adapted processes $\left(x^{i}, r^{i}, y^{i}, p^{i}\right)$ are $\overline{\mathcal{F}}^{0}$-conditionally i.i.d. In particular, for any $\varphi^{i}=$ $x^{i}, r^{i}, y^{i}, p^{i}$, we have $\mathbb{E}_{t}^{0}\left[\varphi_{t}^{i}\right]=\mathbb{E}_{t}^{0}\left[\varphi_{t}^{1}\right]$ and also

$$
\mathbb{E}_{T}^{0}\left[c^{g}\left(c_{T}^{0}, c_{T}^{i}\right) x_{T}^{i}+h^{g}\left(c_{T}^{0}, c_{T}^{i}\right)\right]=\mathbb{E}_{T}^{0}\left[c^{g}\left(c_{T}^{0}, c_{T}^{1}\right) x_{T}^{1}+h^{g}\left(c_{T}^{0}, c_{T}^{1}\right)\right] .
$$

Therefore, $\left(x^{0}, p^{0}\right)$ defined in (5.2) and (5.3) is indeed indistinguishable for every copy.

We are going to compare $\left(x^{0}, p^{0},\left(x^{i}\right)_{i=1}^{N},\left(r^{i}\right)_{i=1}^{N},\left(y^{i}\right)_{i=1}^{N},\left(p^{i}\right)_{i=1}^{N}\right)$ given above with the dynamics $\left(X^{0} / N, P^{0},\left(X^{i}\right)_{i=1}^{N},\left(R^{i}\right)_{i=1}^{N},\left(Y^{i}\right)_{i=1}^{N},\left(P^{i}\right)_{i=1}^{N}\right)$ given by (3.17) and (3.18) with homogeneous coefficients. Using the scaling rule in (3.12) and Remark 3.10, we have for $1 \leq i \leq N$,

$$
\left\{\begin{aligned}
& \mathrm{d} \frac{X_{t}^{0}}{N}=\left\{\overline{\mathcal{V}}_{t}^{0}\left(-P_{t}^{0}+\mathfrak{m}\left(\left(Y_{t}\right)\right)+\mathfrak{m}\left(\left(P_{t}\right)\right)\right)+\mathfrak{l}_{0}\left(t, c_{t}^{0}\right)\right\} \mathrm{d} t+\mathfrak{s}_{0}\left(t, c_{t}^{0}\right) \mathrm{d} W_{t}^{0}, \\
& \mathrm{~d} X_{t}^{i}=\left\{-\bar{\Lambda}_{t}\left(Y_{t}^{i}-\mathfrak{m}\left(\left(Y_{t}\right)\right)-\overline{\mathcal{V}}_{t}^{0}\left(-P_{t}^{0}+\mathfrak{m}\left(\left(Y_{t}\right)\right)+\mathfrak{m}\left(\left(P_{t}\right)\right)\right)+l\left(t, c_{t}^{0}, c_{t}^{i}\right)\right\} \mathrm{d} t\right. \\
&+\sigma^{0}\left(t, c_{t}^{0}, c_{t}^{i}\right) \mathrm{d} W_{t}^{0}+\sigma\left(t, c_{t}^{0}, c_{t}^{i}\right) \mathrm{d} W_{t}^{i}, \\
& \mathrm{~d} R_{t}^{i}=\left\{\bar{\Lambda}_{t}\left(P_{t}^{i}-\mathfrak{m}\left(\left(P_{t}\right)\right)\right)+\overline{\mathcal{V}}_{t}^{0}\left(-P_{t}^{0}+\mathfrak{m}\left(\left(Y_{t}\right)\right)+\mathfrak{m}\left(\left(P_{t}\right)\right)\right)\right\} \mathrm{d} t, \mathrm{~d} P_{t}^{0}=-\partial_{x} \overline{\mathfrak{f}}_{0}\left(t, X_{t}^{0} / N, c_{t}^{0}\right) \mathrm{d} t+Q_{t}^{0,0} \mathrm{~d} W_{t}^{0}+\sum_{j=1}^{N} Q_{t}^{0, j} \mathrm{~d} W_{t}^{j}, \\
& \mathrm{~d} Y_{t}^{i}=-\partial_{x} \bar{f}\left(t, X_{t}^{i}, c_{t}^{0}, c_{t}^{i}\right) \mathrm{d} t+Z_{t}^{i, 0} \mathrm{~d} W_{t}^{0}+\sum_{j=1}^{N} Z_{t}^{i, j} \mathrm{~d} W_{t}^{j}, \\
& \mathrm{~d} P_{t}^{i}=c^{f}\left(t, c_{t}^{0}, c_{t}^{i}\right) R_{t}^{i} \mathrm{~d} t+Q_{t}^{i, 0} \mathrm{~d} W_{t}^{0}+\sum_{j=1}^{N} Q_{t}^{i, j} \mathrm{~d} W_{t}^{j},
\end{aligned}\right.
$$

with

$$
\left\{\begin{array}{l}
X_{0}^{0}=N \chi^{0}, \quad X_{0}^{i}=\xi^{i}, \quad R_{0}^{i}=0 \\
P_{T}^{0}=\partial_{x} \mathfrak{g}_{0}\left(X_{T}^{0} / N, c_{T}^{0}\right), \\
Y_{T}^{i}=\frac{\delta}{1-\delta} \mathfrak{m}\left(\left(c^{g}\left(c_{T}^{0}, c_{T}^{j}\right) X_{T}^{j}+h^{g}\left(c_{T}^{0}, c_{T}^{j}\right)\right)_{j=1}^{N}\right)+c^{g}\left(c_{T}^{0}, c_{T}^{i}\right) X_{T}^{i}+h^{g}\left(c_{T}^{0}, c_{T}^{i}\right) \\
P_{T}^{i}=-c^{g}\left(c_{T}^{0}, c_{T}^{i}\right)\left(R_{T}^{i}+\frac{\delta}{1-\delta} \mathfrak{m}\left(\left(R_{T}\right)\right)\right) .
\end{array}\right.
$$

Thanks to the symmetry, $\left(X^{i}, R^{i}, Y^{i}, P^{i}\right)$ have the same distribution for every $1 \leq i \leq N$, although they are not independent due to their interactions. Let us introduce the notation:

$$
\begin{aligned}
& \Delta x_{t}^{0}:=\frac{X_{t}^{0}}{N}-x_{t}^{0}, \quad \Delta x_{t}^{i}:=X_{t}^{i}-x_{t}^{i}, \quad \Delta r_{t}^{i}:=R_{t}^{i}-r_{t}^{i} \\
& \Delta p_{t}^{0}:=P_{t}^{0}-p_{t}^{0}, \quad \Delta y_{t}^{i}:=Y_{t}^{i}-y_{t}^{i}, \quad \Delta p_{t}^{i}:=P_{t}^{i}-p_{t}^{i} \\
& \Delta q_{t}^{0,0}:=Q_{t}^{0,0}-q_{t}^{0,0}, \quad \Delta q_{t}^{0, j}:=Q_{t}^{0, j} \\
& \Delta z_{t}^{i, 0}:=Z_{t}^{i, 0}-z_{t}^{i, 0}, \quad \Delta z_{t}^{i, j}:=Z_{t}^{i, j}-\delta_{i, j} z_{t}^{i, i} \\
& \Delta q_{t}^{i, 0}:=Q_{t}^{i, 0}-q_{t}^{i, 0}, \quad \Delta q_{t}^{i, j}:=Q_{t}^{i, j}-\delta_{i, j} q_{t}^{i, i}
\end{aligned}
$$

where $\delta_{i, j}$ stands for Kronecker delta. We also define

$$
\begin{aligned}
& \bar{\mu}_{t}^{r, N}:=\frac{1}{N} \sum_{i=1}^{N} \delta_{r_{t}^{i}}, \quad \bar{\mu}_{t}^{y, N}:=\frac{1}{N} \sum_{i=1}^{N} \delta_{y_{t}^{i}}, \quad \bar{\mu}_{t}^{p, N}:=\frac{1}{N} \sum_{i=1}^{N} \delta_{p_{t}^{i}}, \quad \bar{\mu}^{g, N}:=\frac{1}{N} \sum_{i=1}^{N} \delta_{c^{g}\left(c_{T}^{0}, c_{T}^{i}\right) x_{T}^{i}+h^{g}\left(c_{T}^{0}, c_{T}^{i}\right)}, \\
& \mu_{t}^{r}:=\mathcal{L}\left(r_{t}^{1} \mid \overline{\mathcal{F}}_{t}^{0}\right), \quad \mu_{t}^{y}:=\mathcal{L}\left(y_{t}^{1} \mid \overline{\mathcal{F}}_{t}^{0}\right), \quad \mu_{t}^{p}:=\mathcal{L}\left(p_{t}^{1} \mid \overline{\mathcal{F}}_{t}^{0}\right), \quad \mu^{g}:=\mathcal{L}\left(c^{g}\left(c_{T}^{0}, c_{T}^{1}\right) x_{T}^{1}+\left.h^{g}\left(c_{T}^{0}, c_{T}^{1}\right)\right|^{\mathcal{F}}{ }_{T}^{0}\right) .
\end{aligned}
$$


Here, $\bar{\mu}^{r, N}, \bar{\mu}^{y, N}, \bar{\mu}^{p, N}$ and $\bar{\mu}^{g, N}$ denote the empirical measures, and the others conditional distributions. When the filtration defined on the product space is completed, there appears some subtle issue on the conditional distribution about its measurability. However, we can always construct a measurable version by modifying it only on the null sets. We always suppose that $\left(\mu^{r}, \mu^{y}, \mu^{p}, \mu^{g}\right)$ are measurable versions constructed in such a way. See Section 2.1.3 in [11] for details. Since $\left(r^{i}, y^{i}, p^{i}, c^{g}\left(c_{T}^{0}, c_{T}^{i}\right) x_{T}^{i}+h^{g}\left(c_{T}^{0}, c_{T}^{i}\right)\right), 1 \leq i \leq N$ are $\overline{\mathcal{F}}^{0}$ conditionally i.i.d. and also $\left(r^{i}, y^{i}, p^{i}\right)$ are continuous processes, we have the following convergence properties.

Lemma 5.2. Let Assumption (Minor-Homogeneous) be in force. Then we have

$$
\begin{aligned}
& \lim _{N \rightarrow \infty} \sup _{t \in[0, T]} \mathbb{E}\left[W_{2}\left(\bar{\mu}_{t}^{r, N}, \mu_{t}^{r}\right)^{2}+W_{2}\left(\bar{\mu}_{t}^{y, N}, \mu_{t}^{y}\right)^{2}+W_{2}\left(\bar{\mu}_{t}^{p, N}, \mu_{t}^{p}\right)^{2}\right]=0, \\
& \lim _{N \rightarrow \infty} \mathbb{E}\left[W_{2}\left(\mu^{g, N}, \mu^{g}\right)^{2}\right]=0 .
\end{aligned}
$$

Moreover, if there exist some positive constants $\Gamma$ and $\Gamma_{g}$ such that $\sup _{t \in[0, T]}\left(\mathbb{E}\left[\left|r_{t}^{1}\right|^{k}\right]^{\frac{1}{k}}+\mathbb{E}\left[\left|y_{t}^{1}\right|^{k}\right]^{\frac{1}{k}}+\mathbb{E}\left[\left|p_{t}^{1}\right|^{k}\right]^{\frac{1}{k}}\right) \leq$ $\Gamma$ and $\mathbb{E}\left[\left|c^{g}\left(c_{T}^{0}, c_{T}^{1}\right) x_{T}^{1}+h^{g}\left(c_{T}^{0}, c_{T}^{1}\right)\right|^{k}\right]^{\frac{1}{k}} \leq \Gamma_{g}$ for some $k>4$, then there exists some constant $C$ independent of $N$ such that

$$
\begin{aligned}
& \sup _{t \in[0, T]} \mathbb{E}\left[W_{2}\left(\bar{\mu}_{t}^{r, N}, \mu_{t}^{r}\right)^{2}+W_{2}\left(\bar{\mu}_{t}^{y, N}, \mu_{t}^{y}\right)^{2}+W_{2}\left(\bar{\mu}_{t}^{p, N}, \mu_{t}^{p}\right)^{2}\right] \leq C \Gamma^{2} \epsilon_{N}, \\
& \quad \mathbb{E}\left[W_{2}\left(\bar{\mu}^{g, N}, \mu^{g}\right)^{2}\right] \leq C \Gamma_{g}^{2} \epsilon_{N}
\end{aligned}
$$

with $\epsilon_{N}:=N^{-2 / \max (n, 4)}\left(1+\log (N) \mathbf{1}_{N=4}\right)$.

Proof. See Lemma 4.1 in [28] and the proof for Theorem 5.1 in [27]. More details on the Glivenko-Cantelli convergence in the Wasserstein distance are available from Section 5.1 in [10] and references therein.

The next property of the Wasserstein distance is important for our purpose. For any $\mu, \nu \in \mathcal{P}_{2}\left(\mathbb{R}^{n}\right)$, it is easy to check

$$
\left|\int_{\mathbb{R}^{n}} x \mu(\mathrm{d} x)-\int_{\mathbb{R}^{n}} y \nu(\mathrm{d} y)\right|=\left|\int_{\mathbb{R}^{n \times n}}(x-y) \pi(\mathrm{d} x, \mathrm{~d} y)\right| \leq \int_{\mathbb{R}^{n \times n}}|x-y| \pi(\mathrm{d} x, \mathrm{~d} y)
$$

for any coupling $\pi \in \Pi_{2}(\mu, \nu)$ with marginals $\mu$ and $\nu$. Taking infimum over $\pi \in \Pi_{2}(\mu, \nu)$, we get

$$
\left|\int_{\mathbb{R}^{n}} x \mu(\mathrm{d} x)-\int_{\mathbb{R}^{n}} y \nu(\mathrm{d} y)\right| \leq W_{1}(\mu, \nu) \leq W_{2}(\mu, \nu) .
$$

We are now ready to prove the main result of this section.

Theorem 5.3. Let Assumption (Minor-Homogeneous) be in force. Then, for every $1 \leq i \leq N$, there exists an $N$-independent constant $C$ such that

$$
\begin{aligned}
& \mathbb{E}\left[\sup _{t \in[0, T]}\left(\left|\Delta x_{t}^{0}\right|^{2}+\left|\Delta x_{t}^{i}\right|^{2}+\left|\Delta r_{t}^{i}\right|^{2}+\left|\Delta p_{t}^{0}\right|^{2}+\left|\Delta y_{t}^{i}\right|^{2}+\left|\Delta p_{t}^{i}\right|^{2}\right)\right. \\
& \left.\quad+\sum_{j=0}^{N} \int_{0}^{T}\left(\left|\Delta q_{t}^{0, j}\right|^{2}+\left|\Delta z_{t}^{i, j}\right|^{2}+\left|\Delta q_{t}^{i, j}\right|^{2}\right) \mathrm{d} t\right] \\
& \leq C \mathbb{E}\left[W_{2}\left(\bar{\mu}^{g, N}, \mu^{g}\right)^{2}+W_{2}\left(\bar{\mu}_{T}^{r, N}, \mu_{T}^{r}\right)^{2}+\int_{0}^{T}\left(W_{2}\left(\bar{\mu}_{t}^{y, N}, \mu_{t}^{y}\right)^{2}+W_{2}\left(\bar{\mu}_{t}^{p, N}, \mu_{t}^{p}\right)^{2}\right) \mathrm{d} t\right] .
\end{aligned}
$$


Proof. Let us define $\gamma>0$ by

$$
\gamma:=\min \left\{\gamma_{0}^{f}, \gamma^{f}, \gamma_{0}^{g}, \gamma^{g}-\mathfrak{a}\right\}
$$

First step: We want to apply Itô-formula to

$$
\left(\left\langle\Delta p_{t}^{0}, \Delta x_{t}^{0}\right\rangle+\frac{1}{N} \sum_{i=1}^{N}\left\langle\Delta y_{t}^{i}, \Delta x_{t}^{i}\right\rangle+\frac{1}{N} \sum_{i=1}^{N}\left\langle\Delta p_{t}^{i},(-I) \Delta r_{t}^{i}\right\rangle\right)
$$

With this in mind, we check the following estimates. It is easy to see, with obvious notation,

$$
\begin{aligned}
& \left\langle\operatorname{drift}\left[\Delta p_{t}^{0}\right], \Delta x_{t}^{0}\right\rangle+\frac{1}{N} \sum_{i=1}^{N}\left\langle\operatorname{drift}\left[\Delta y_{t}^{i}\right], \Delta x_{t}^{i}\right\rangle+\frac{1}{N} \sum_{i=1}^{N}\left\langle\operatorname{drift}\left[\Delta p_{t}^{i}\right],(-I) \Delta r_{t}^{i}\right\rangle \\
& \leq-\gamma_{0}^{f}\left|\Delta x_{t}^{0}\right|^{2}-\gamma^{f} \frac{1}{N} \sum_{i=1}^{N}\left(\left|\Delta x_{t}^{i}\right|^{2}+\left|\Delta r_{t}^{i}\right|^{2}\right)
\end{aligned}
$$

Using (5.6), we get

$$
\begin{aligned}
\left\langle\operatorname{drift}\left[\Delta x_{t}^{0}\right], \Delta p_{t}^{0}\right\rangle=\left\langle\overline{\mathcal{V}}_{t}^{0}\left(-\Delta p_{t}^{0}+\mathfrak{m}\left(\left(Y_{t}\right)\right)-\mathbb{E}_{t}^{0}\left[y_{t}^{1}\right]+\mathfrak{m}\left(\left(P_{t}\right)\right)-\mathbb{E}_{t}^{0}\left[p_{t}^{1}\right]\right), \Delta p_{t}^{0}\right\rangle \\
=\left\langle\overline{\mathcal{V}}_{t}^{0}\left(-\Delta p_{t}^{0}+\mathfrak{m}\left(\left(\Delta y_{t}\right)\right)+\mathfrak{m}\left(\left(\Delta p_{t}\right)\right)\right), \Delta p_{t}^{0}\right\rangle \\
\quad+\left\langle\overline{\mathcal{V}}_{t}^{0}\left(\mathfrak{m}\left(\left(y_{t}\right)\right)-\mathbb{E}_{t}^{0}\left[y_{t}^{1}\right]+\mathfrak{m}\left(\left(p_{t}\right)\right)-\mathbb{E}_{t}^{0}\left[p_{t}^{1}\right]\right), \Delta p_{t}^{0}\right\rangle \\
\leq\left\langle\overline{\mathcal{V}}_{t}^{0}\left(-\Delta p_{t}^{0}+\mathfrak{m}\left(\left(\Delta y_{t}\right)\right)+\mathfrak{m}\left(\left(\Delta p_{t}\right)\right)\right), \Delta p_{t}^{0}\right\rangle \\
\quad+C\left(W_{2}\left(\bar{\mu}_{t}^{y, N}, \mu_{t}^{y}\right)+W_{2}\left(\bar{\mu}_{t}^{p, N}, \mu_{t}^{p}\right)\right)\left|\Delta p_{t}^{0}\right|
\end{aligned}
$$

Similar calculation yields

$$
\begin{aligned}
\langle\operatorname{drift} & {\left.\left[\Delta x_{t}^{0}\right], \Delta p_{t}^{0}\right\rangle+\frac{1}{N} \sum_{i=1}^{N}\left\langle\operatorname{drift}\left[\Delta x_{t}^{i}\right], \Delta y_{t}^{i}\right\rangle+\frac{1}{N} \sum_{i=1}^{N}\left\langle\operatorname{drift}\left[\Delta r_{t}^{i}\right],(-I) \Delta p_{t}^{i}\right\rangle } \\
\leq & -\left\langle\overline{\mathcal{V}}_{t}^{0}\left(-\Delta p_{t}^{0}+\mathfrak{m}\left(\left(\Delta y_{t}\right)\right)+\mathfrak{m}\left(\left(\Delta p_{t}\right)\right)\right),-\Delta p_{t}^{0}+\mathfrak{m}\left(\left(\Delta y_{t}\right)\right)+\mathfrak{m}\left(\left(\Delta p_{t}\right)\right)\right\rangle \\
& +C\left(W_{2}\left(\bar{\mu}_{t}^{y, N}, \mu_{t}^{y}\right)+W_{2}\left(\bar{\mu}_{t}^{p, N}, \mu_{t}^{p}\right)\right)\left(\left|\Delta p_{t}^{0}\right|+\left|\mathfrak{m}\left(\left(\Delta y_{t}\right)\right)\right|+\left|\mathfrak{m}\left(\left(\Delta p_{t}\right)\right)\right|\right) \\
\leq & C\left(W_{2}\left(\bar{\mu}_{t}^{y, N}, \mu_{t}^{y}\right)+W_{2}\left(\bar{\mu}_{t}^{p, N}, \mu_{t}^{p}\right)\right)\left(\left|\Delta p_{t}^{0}\right|+\mathfrak{m}\left(\left(\left|\Delta y_{t}\right|\right)\right) \mid+\mathfrak{m}\left(\left(\left|\Delta p_{t}\right|\right)\right)\right) .
\end{aligned}
$$

Now, let us check the terminal parts. Similar analysis used in (3.9) yields

$$
\begin{aligned}
& \left\langle\Delta p_{T}^{0}, \Delta x_{T}^{0}\right\rangle+\frac{1}{N} \sum_{i=1}^{N}\left\langle\Delta y_{T}^{i}, \Delta x_{T}^{i}\right\rangle+\frac{1}{N} \sum_{i=1}^{N}\left\langle\Delta p_{T}^{i},(-I) \Delta r_{T}^{i}\right\rangle \\
& \geq \gamma_{0}^{g}\left|\Delta x_{T}^{0}\right|^{2}+\left(\gamma^{g}-\mathfrak{a}\right) \frac{1}{N} \sum_{i=1}^{N}\left(\left|\Delta x_{T}^{i}\right|^{2}+\left|\Delta r_{T}^{i}\right|^{2}\right) \\
& \quad-C W_{2}\left(\bar{\mu}^{g, N}, \mu^{g}\right) \mathfrak{m}\left(\left(\left|\Delta x_{T}\right|\right)\right)-C W_{2}\left(\bar{\mu}_{T}^{r, N}, \mu_{T}^{r}\right) \mathfrak{m}\left(\left(\left|\Delta r_{T}\right|\right)\right) .
\end{aligned}
$$


From (5.8) and (5.9), we get

$$
\begin{aligned}
& \mathbb{E}\left[\int_{0}^{T} d\left(\left\langle\Delta p_{t}^{0}, \Delta x_{t}^{0}\right\rangle+\frac{1}{N} \sum_{i=1}^{N}\left\langle\Delta y_{t}^{i}, \Delta x_{t}^{i}\right\rangle+\frac{1}{N} \sum_{i=1}^{N}\left\langle\Delta p_{t}^{i},(-I) \Delta r_{t}^{i}\right\rangle\right)\right] \\
& \leq-\gamma \mathbb{E}\left[\int_{0}^{T}\left(\left|\Delta x_{t}^{0}\right|^{2}+\frac{1}{N} \sum_{i=1}^{N}\left(\left|\Delta x_{t}^{i}\right|^{2}+\left|\Delta r_{t}^{i}\right|^{2}\right)\right) \mathrm{d} t\right] \\
& \quad+C \mathbb{E}\left[\int_{0}^{T}\left(W_{2}\left(\bar{\mu}_{t}^{y, N}, \mu_{t}^{y}\right)+W_{2}\left(\bar{\mu}_{t}^{p, N}, \mu_{t}^{p}\right)\right)\left(\left|\Delta p_{t}^{0}\right|+\mathfrak{m}\left(\left(\left|\Delta y_{t}\right|\right)\right)+\mathfrak{m}\left(\left(\left|\Delta p_{t}\right|\right)\right)\right) \mathrm{d} t\right] .
\end{aligned}
$$

Note that there is no quadratic covariation term. Now combining the estimate (5.10), we obtain the following:

$$
\begin{gathered}
\gamma \mathbb{E}\left[\left|\Delta x_{T}^{0}\right|^{2}+\frac{1}{N} \sum_{i=1}^{N}\left(\left|\Delta x_{T}^{i}\right|^{2}+\left|\Delta r_{T}^{i}\right|^{2}\right)+\int_{0}^{T}\left(\left|\Delta x_{t}^{0}\right|^{2}+\frac{1}{N} \sum_{i=1}^{N}\left(\left|\Delta x_{t}^{i}\right|^{2}+\left|\Delta r_{t}^{i}\right|^{2}\right)\right) \mathrm{d} t\right] \\
\leq C \mathbb{E}\left[W_{2}\left(\bar{\mu}^{g, N}, \mu^{g}\right) \mathfrak{m}\left(\left(\left|\Delta x_{T}\right|\right)\right)+W_{2}\left(\bar{\mu}_{T}^{r, N}, \mu_{T}^{r}\right) \mathfrak{m}\left(\left(\left|\Delta r_{T}\right|\right)\right)\right. \\
\left.\quad+\int_{0}^{T}\left(W_{2}\left(\bar{\mu}_{t}^{y, N}, \mu_{t}^{y}\right)+W_{2}\left(\bar{\mu}_{t}^{p, N}, \mu_{t}^{p}\right)\right)\left(\left|\Delta p_{t}^{0}\right|+\mathfrak{m}\left(\left(\left|\Delta y_{t}\right|\right)\right)+\mathfrak{m}\left(\left(\left|\Delta p_{t}\right|\right)\right)\right) \mathrm{d} t\right] .
\end{gathered}
$$

By Young's inequality and the symmetry of the distribution, we find that the following inequality holds for every $1 \leq i \leq N$ :

$$
\begin{aligned}
\mathbb{E}\left[\left|\Delta x_{T}^{0}\right|^{2}+\left|\Delta x_{T}^{i}\right|^{2}+\left|\Delta r_{T}^{i}\right|^{2}+\int_{0}^{T}\left(\left|\Delta x_{t}^{0}\right|^{2}+\left|\Delta x_{t}^{i}\right|^{2}+\left|\Delta r_{t}^{i}\right|^{2}\right) \mathrm{d} t\right] \\
\leq C \mathbb{E}\left[W_{2}\left(\bar{\mu}^{g, N}, \mu^{g}\right)^{2}+W_{2}\left(\bar{\mu}_{T}^{r, N}, \mu_{T}^{r}\right)^{2}\right. \\
\left.\quad+\int_{0}^{T}\left(W_{2}\left(\bar{\mu}_{t}^{y, N}, \mu_{t}^{y}\right)+W_{2}\left(\bar{\mu}_{t}^{p, N}, \mu_{t}^{p}\right)\right)\left(\left|\Delta p_{t}^{0}\right|+\mathfrak{m}\left(\left(\left|\Delta y_{t}\right|\right)\right)+\mathfrak{m}\left(\left(\left|\Delta p_{t}\right|\right)\right)\right) \mathrm{d} t\right] .
\end{aligned}
$$

\section{Second step}

From the standard estimate of the BSDEs, see Section 4.4 in [56] for example, it is easy to find

$$
\begin{aligned}
& \left.\mathbb{E}_{t \in[0, T]} \sup _{t \in}\left|\Delta p_{t}^{0}\right|^{2}+\int_{0}^{T}\left(\left|\Delta q_{t}^{0,0}\right|^{2}+\sum_{j=1}^{N}\left|\Delta q_{t}^{0, j}\right|^{2}\right) \mathrm{d} t\right] \\
& \leq C \mathbb{E}\left[\left|\Delta p_{T}^{0}\right|^{2}+\int_{0}^{T}\left|\partial_{x} \overline{\mathfrak{f}}_{0}\left(t, X_{t}^{0} / N, c_{t}^{0}\right)-\overline{\mathfrak{f}}_{0}\left(t, x_{t}^{0}, c_{t}^{0}\right)\right|^{2} \mathrm{~d} t\right] \\
& \leq C \mathbb{E}\left[\left|\Delta x_{T}^{0}\right|^{2}+\int_{0}^{T}\left|\Delta x_{t}^{0}\right|^{2} \mathrm{~d} t\right] .
\end{aligned}
$$


Carrying out the similar analysis for $\left(\Delta y^{i}, \Delta p^{i}\right)$ and using the symmetry among $1 \leq i \leq N$, we obtain for any $1 \leq i \leq N$,

$$
\begin{aligned}
\mathbb{E}\left[\sup _{t \in[0, T]}\left(\left|\Delta p_{t}^{0}\right|^{2}+\left|\Delta y_{t}^{i}\right|^{2}+\left|\Delta p_{t}^{i}\right|^{2}\right)+\sum_{j=0}^{N} \int_{0}^{T}\left(\left|\Delta q_{t}^{0, j}\right|^{2}+\left|\Delta z_{t}^{i, j}\right|^{2}+\left|\Delta q_{t}^{i, j}\right|^{2}\right) \mathrm{d} t\right] \\
\leq C \mathbb{E}\left[\left|\Delta x_{T}^{0}\right|^{2}+\left|\Delta x_{T}^{i}\right|^{2}+\left|\Delta r_{T}^{i}\right|^{2}+\int_{0}^{T}\left(\left|\Delta x_{t}^{0}\right|^{2}+\left|\Delta x_{t}^{i}\right|^{2}+\left|\Delta r_{t}^{i}\right|^{2}\right) \mathrm{d} t\right] \\
\quad+C \mathbb{E}\left[W_{2}\left(\bar{\mu}^{g, N}, \mu^{g}\right)^{2}+W_{2}\left(\bar{\mu}_{T}^{r, N}, \mu_{T}^{r}\right)^{2}\right] \\
\leq C \mathbb{E}\left[W_{2}\left(\bar{\mu}^{g, N}, \mu^{g}\right)^{2}+W_{2}\left(\bar{\mu}_{T}^{r, N}, \mu_{T}^{r}\right)^{2}\right. \\
\left.\quad+\int_{0}^{T}\left(W_{2}\left(\bar{\mu}_{t}^{y, N}, \mu_{t}^{y}\right)+W_{2}\left(\bar{\mu}_{t}^{p, N}, \mu_{t}^{p}\right)\right)\left(\left|\Delta p_{t}^{0}\right|+\mathfrak{m}\left(\left(\left|\Delta y_{t}\right|\right)\right)+\mathfrak{m}\left(\left(\left|\Delta p_{t}\right|\right)\right)\right) \mathrm{d} t\right],
\end{aligned}
$$

where we have used (5.11) in the second inequality.

From (5.11), (5.12) and the symmetry among $1 \leq i \leq N$, Young's inequality yields

$$
\begin{aligned}
& \mathbb{E}\left[\left|\Delta x_{T}^{0}\right|^{2}+\left|\Delta x_{T}^{i}\right|^{2}+\left|\Delta r_{T}^{i}\right|^{2}+\int_{0}^{T}\left(\left|\Delta x_{t}^{0}\right|^{2}+\left|\Delta x_{t}^{i}\right|^{2}+\left|\Delta r_{t}^{i}\right|^{2}\right) \mathrm{d} t\right] \\
& \quad+\mathbb{E}\left[\sup _{t \in[0, T]}\left(\left|\Delta p_{t}^{0}\right|^{2}+\left|\Delta y_{t}^{i}\right|^{2}+\left|\Delta p_{t}^{i}\right|^{2}\right)+\sum_{j=0}^{N} \int_{0}^{T}\left(\left|\Delta q_{t}^{0, j}\right|^{2}+\left|\Delta z_{t}^{i, j}\right|^{2}+\left|\Delta q_{t}^{i, j}\right|^{2}\right) \mathrm{d} t\right] \\
& \leq C \mathbb{E}\left[W_{2}\left(\bar{\mu}^{g, N}, \mu^{g}\right)^{2}+W_{2}\left(\bar{\mu}_{T}^{r, N}, \mu_{T}^{r}\right)^{2}+\int_{0}^{T}\left(W_{2}\left(\bar{\mu}_{t}^{y, N}, \mu_{t}^{y}\right)^{2}+W_{2}\left(\bar{\mu}_{t}^{p, N}, \mu_{t}^{p}\right)^{2}\right) \mathrm{d} t\right] .
\end{aligned}
$$

Now the desired estimate follows from a simple application of Burkholder-Davis-Gundy (BDG) inequality to the forward variables $\left(\Delta x^{0}, \Delta x^{i}, \Delta r^{i}\right)$.

\subsection{Some stability results}

For understanding the implications of Lemma 5.2 and Theorem 5.3, let us denote the market-clearing price for the $N$ homogeneous minor agents by

$$
\varpi_{t}^{\mathrm{Ho}, N}:=-\mathfrak{m}\left(\left(Y_{t}\right)\right)+\overline{\mathcal{V}}_{t}^{0}\left(-P_{t}^{0}+\mathfrak{m}\left(\left(Y_{t}\right)\right)+\mathfrak{m}\left(\left(P_{t}\right)\right)\right), \quad t \in[0, T)
$$

using the solution to (5.4) with (5.5). By comparing it with $\varpi_{t}^{\mathrm{mfg}}$ in (5.1), we get the following result.

Theorem 5.4. Under Assumption (Minor-Homogeneous), the following inequality holds:

$$
\begin{aligned}
& \mathbb{E} \int_{0}^{T}\left|\varpi_{t}^{\mathrm{Ho}, N}-\varpi_{t}^{\mathrm{mfg}}\right|^{2} \mathrm{~d} t \\
& \quad \leq C \mathbb{E}\left[W_{2}\left(\bar{\mu}^{g, N}, \mu^{g}\right)^{2}+W_{2}\left(\bar{\mu}_{T}^{r, N}, \mu_{T}^{r}\right)^{2}+\int_{0}^{T}\left(W_{2}\left(\bar{\mu}_{t}^{y, N}, \mu_{t}^{y}\right)^{2}+W_{2}\left(\bar{\mu}_{t}^{p, N}, \mu_{t}^{p}\right)^{2}\right) \mathrm{d} t\right]
\end{aligned}
$$


where $C$ is some positive constant independent of $N$.

Proof. Using the symmetry, we have

$$
\mathbb{E}\left[\left|\varpi_{t}^{\mathrm{Ho}, N}-\varpi_{t}^{\mathrm{mfg}}\right|^{2}\right] \leq C \mathbb{E}\left[\left|\Delta p_{t}^{0}\right|^{2}+\left|\Delta y_{t}^{1}\right|^{2}+\left|\Delta p_{t}^{1}\right|^{2}+W_{2}\left(\bar{\mu}_{t}^{y, N}, \mu_{t}^{y}\right)^{2}+W_{2}\left(\bar{\mu}_{t}^{p, N}, \mu_{t}^{p}\right)^{2}\right]
$$

Hence Theorem 5.3 gives the desired estimate.

From Lemma 5.2, we observe that $\left(\varpi_{t}^{\mathrm{Ho}, N}\right)_{t \in[0, T]}$ converges to $\left(\varpi_{t}^{\mathrm{mfg}}\right)_{t \in[0, T]}$ in the large population limit of homogeneous minor agents. In this limit, the optimization problem for each $i$ th minor agent given in (3.5) is solved within $\left(\Omega^{i}, \mathcal{F}^{i}, \mathbb{P}^{i} ; \mathbb{F}^{i}\right)$ since the market price process $\varpi^{\mathrm{mfg}}$ is now $\overline{\mathbb{F}}^{0}$-adapted i.e. dependent only on the common market information. One can observe that the natural information structure mentioned in Remark 3.1 is actually achieved in the mean-field limit.

Before closing the paper, let us briefly discuss about the stability relation between the heterogeneous and the homogeneous market. Let $\left(X^{0},\left(X^{i}\right)_{i=1}^{N},\left(R^{i}\right)_{i=1}^{N}, P^{0},\left(P^{i}\right)_{i=1}^{N}\right)$ denote the unique solution to (3.17) with (3.18) given by Theorem 3.14 in the market with heterogeneous minor agents, and $\left(\underline{X}^{0},\left(\underline{X}^{i}\right)_{i=1}^{N},\left(\underline{R}^{i}\right)_{i=1}^{N}, \underline{P}^{0},\left(\underline{P}^{i}\right)_{i=1}^{N}\right)$ the unique solution to (5.4) with (5.5) corresponding to the homogeneous minor agents. Let us introduce the following notation: for $1 \leq i \leq N$,

$$
\begin{aligned}
& \delta l_{i}(t):=l_{i}\left(t, c_{t}^{0}, c_{t}^{i}\right)-l\left(t, c_{t}^{0}, c_{t}^{i}\right), \\
& \delta \sigma_{i}^{0}(t):=\sigma_{i}^{0}\left(t, c_{t}^{0}, c_{t}^{i}\right)-\sigma^{0}\left(t, c_{t}^{0}, c_{t}^{i}\right), \quad \delta \sigma_{i}(t):=\sigma_{i}\left(t, c_{t}^{0}, c_{t}^{i}\right)-\sigma\left(t, c_{t}^{0}, c_{t}^{i}\right), \\
& \delta \partial_{x} \bar{f}_{i}(t):=\partial_{x} \bar{f}_{i}\left(t, \underline{X}_{t}^{i}, c_{t}^{0}, c_{t}^{i}\right)-\partial_{x} \bar{f}\left(t, \underline{X}_{t}^{i}, c_{t}^{0}, c_{t}^{i}\right), \\
& \delta c_{i}^{f}(t):=c_{i}^{f}\left(t, c_{t}^{0}, c_{t}^{i}\right)-c^{f}\left(t, c_{t}^{0}, c_{t}^{i}\right), \\
& \delta h_{i}^{g}=h_{i}^{g}\left(c_{T}^{0}, c_{T}^{i}\right)-h^{g}\left(c_{T}^{0}, c_{T}^{i}\right) .
\end{aligned}
$$

Denoting the market-clearing price (3.21) in the market with $N$ heterogeneous agents by $\left(\varpi_{t}^{\mathrm{He}, N}\right)_{t \in[0, T)}$, we have the next stability result.

Corollary 5.5. Let Assumptions (Minor-A, B) and (MFG) be in force. Then the following inequality holds:

$$
\begin{aligned}
& \mathbb{E} \int_{0}^{T}\left|\varpi_{t}^{\mathrm{He}, N}-\varpi_{t}^{\mathrm{mfg}}\right|^{2} \mathrm{~d} t \\
& \quad \leq C \mathbb{E}\left[W_{2}\left(\bar{\mu}^{g, N}, \mu^{g}\right)^{2}+W_{2}\left(\bar{\mu}_{T}^{r, N}, \mu_{T}^{r}\right)^{2}+\int_{0}^{T}\left(W_{2}\left(\bar{\mu}_{t}^{y, N}, \mu_{t}^{y}\right)^{2}+W_{2}\left(\bar{\mu}_{t}^{p, N}, \mu_{t}^{p}\right)^{2}\right) \mathrm{d} t\right] \\
& \quad+C \frac{1}{N} \sum_{i=1}^{N} \mathbb{E} \int_{0}^{T}\left(\left|\partial_{x} \bar{f}_{i}(t)\right|^{2}+\left|\delta c_{i}^{f}(t) \underline{R}_{t}^{i}\right|^{2}+\left|\delta l_{i}(t)\right|^{2}+\left|\delta \sigma_{i}^{0}(t)\right|^{2}+\left|\delta \sigma_{i}(t)\right|^{2}\right) \mathrm{d} t \\
& \quad+C \frac{1}{N} \sum_{i=1}^{N} \mathbb{E}\left[\left|\delta c_{i}^{g} \underline{X}_{T}^{i}+\delta h_{i}^{g}\right|^{2}+\left|\delta c_{i}^{g}\left(\underline{R}_{T}^{i}+\frac{\delta}{1-\delta} \mathfrak{m}\left(\left(\underline{R}_{T}\right)\right)\right)\right|^{2}\right] .
\end{aligned}
$$


Proof. Let us put $\Delta X_{t}^{0}:=X_{t}^{0}-\underline{X}_{t}^{0}, \Delta Y_{t}^{i}=Y_{t}^{i}-\underline{Y}_{t}^{i}$, and similarly for the others. Thanks to the stability of fully-coupled FBSDEs, see for example Proposition 3.1 in [28] or more generally Proposition 3.4 in [53], we have

$$
\begin{aligned}
\frac{1}{N} \sum_{i=1}^{N} \mathbb{E} & {\left[\sup _{t \in[0, T]}\left(\left|\frac{\Delta X_{t}^{0}}{N}\right|^{2}+\left|\Delta X_{t}^{i}\right|^{2}+\left|\Delta R_{t}^{i}\right|^{2}+\left|\Delta P_{t}^{0}\right|^{2}+\left|\Delta Y_{t}^{i}\right|^{2}+\left|\Delta P_{t}^{i}\right|^{2}\right)\right.} \\
& \left.+\sum_{j=0}^{N} \int_{0}^{T}\left(\left|\Delta Q_{t}^{0, j}\right|^{2}+\left|\Delta Z_{t}^{i, j}\right|^{2}+\left|\Delta Q_{t}^{i, j}\right|^{2}\right) \mathrm{d} t\right] \\
\leq & C \frac{1}{N} \sum_{i=1}^{N} \mathbb{E} \int_{0}^{T}\left(\left|\delta \partial_{x} \bar{f}_{i}(t)\right|^{2}+\left|\delta c_{i}^{f}(t) \underline{R}_{t}^{i}\right|^{2}+\left|\delta l_{i}(t)\right|^{2}+\left|\delta \sigma_{i}^{0}(t)\right|^{2}+\left|\delta \sigma_{i}(t)\right|^{2}\right) \mathrm{d} t \\
+C & \frac{1}{N} \sum_{i=1}^{N} \mathbb{E}\left[\left|\delta c_{i}^{g} \underline{X}_{T}^{i}+\delta h_{i}^{g}\right|^{2}+\left|\delta c_{i}^{g}\left(\underline{R}_{T}^{i}+\frac{\delta}{1-\delta} \mathfrak{m}\left(\left(\underline{R}_{T}\right)\right)\right)\right|^{2}\right] .
\end{aligned}
$$

Since

$$
\mathbb{E}\left|\varpi_{t}^{\mathrm{He}, N}-\varpi_{t}^{\mathrm{Ho}, N}\right|^{2} \leq C \mathbb{E}\left[\left|\Delta P_{t}^{0}\right|^{2}+\frac{1}{N} \sum_{i=1}^{N}\left(\left|\Delta Y_{t}^{i}\right|^{2}+\left|\Delta P_{t}^{i}\right|^{2}\right)\right]
$$

the estimate (5.13) and Theorem 5.4 give the desired inequality.

\subsection{Mean-field limit as an approximation}

By the MFG theory for the standard Nash-game settings, it is well-known that the equilibrium strategy in the mean-field limit provides an $\epsilon_{N}$-Nash equilibrium for the corresponding finite $N$-agent game [10, 11]. For the market-clearing equilibrium, the results of this section allow us to obtain not only the accuracy of the approximation but also the strong convergence in the large $N$-limit. In fact, Theorem 5.4 combined with Lemma 5.2 provides not only the accuracy of $\varpi^{\mathrm{mfg}}$ as an approximation but also the convergence speed of the true price process $\varpi^{\mathrm{Ho}, N}$ in the finite (homogeneous) agent market.

Let us also mention about the trading strategy in the equilibrium. As one can imagine, the equilibrium strategy in the mean field limit

$$
\begin{aligned}
& \widehat{\beta}_{t}^{\mathrm{mfg}}:=\overline{\mathcal{V}}_{t}^{0}\left(-p_{t}^{0}+\mathbb{E}_{t}^{0}\left[y_{t}^{1}\right]+\mathbb{E}_{t}^{0}\left[p_{t}^{1}\right]\right), \\
& \widehat{\alpha}_{t}^{\mathrm{mfg}, i}:=-\bar{\Lambda}_{t}\left(y_{t}^{i}-\mathbb{E}_{t}^{0}\left[y_{t}^{i}\right]\right)-\overline{\mathcal{V}}_{t}^{0}\left(-p_{t}^{0}+\mathbb{E}_{t}^{0}\left[y_{t}^{i}\right]+\mathbb{E}_{t}^{0}\left[p_{t}^{i}\right]\right), \quad 1 \leq i \leq N
\end{aligned}
$$

gives an approximate strategy for the finite agent market, where $\overline{\mathcal{V}}_{t}^{0}=\left(\Lambda^{0}+2 \Lambda_{t}\right)^{-1}$ and $\left(p^{0}, y^{i}, p^{i}\right)$ is the solution to the McKean-Vlasov FBSDE (5.2). Note that $\varphi^{i}=x^{i}, r^{i}, y^{i}, p^{i}$, we have $\mathbb{E}_{t}^{0}\left[\varphi_{t}^{i}\right]=\mathbb{E}_{t}^{0}\left[\varphi_{t}^{1}\right]$ for every $1 \leq i \leq N$ and more importantly that $\widehat{\beta}^{\mathrm{mfg}}$ and $\widehat{\alpha}^{\mathrm{mfg}, i}$ are $\overline{\mathbb{F}}^{0}$ - and $\mathbb{F}^{i}$-adapted, respectively. This means that each agent can implement an approximate strategy without knowing the idiosyncratic information for the other agents. From the result of Section 3, the true equilibrium strategy in the $N$-agent (homogeneous) market is given by

$$
\begin{aligned}
& \widehat{\beta}_{t}^{N} / N:=\overline{\mathcal{V}}_{t}^{0}\left(-P_{t}^{0}+\mathfrak{m}\left(\left(Y_{t}\right)\right)+\mathfrak{m}\left(\left(P_{t}\right)\right)\right), \\
& \widehat{\alpha}_{t}^{N, i}:=-\bar{\Lambda}_{t}\left(Y_{t}^{i}-\mathfrak{m}\left(\left(Y_{t}\right)\right)\right)-\overline{\mathcal{V}}_{t}^{0}\left(-P_{t}^{0}+\mathfrak{m}\left(\left(Y_{t}\right)\right)+\mathfrak{m}\left(\left(P_{t}\right)\right)\right), 1 \leq i \leq N
\end{aligned}
$$


where $\left(P^{0}, Y^{i}, P^{i}\right), 1 \leq i \leq N$ is the solution to the $N$-coupled system of FBSDEs (5.4). Observe that every agent needs the perfect information $\mathbb{F}$ to implement the strategy in this case. The accuracy of $\left(\widehat{\beta}_{t}^{\mathrm{mfg}}, \widehat{\alpha}_{t}^{\mathrm{mfg}, i}\right)$ as an approximation for the true strategy $\left(\widehat{\beta}_{t}^{N} / N, \widehat{\alpha}_{t}^{N, i}\right)$ can be derived from Theorem 5.3. In fact, the estimate of the square difference becomes essentially the same as for the equilibrium price process. In the case of heterogeneous minor agents, one can make use of the stability property of FBSDE as in Corollary 5.5.

Although it is difficult to obtain a numerical solution for McKean-Vlasov FBSDE (5.2), it looks at least more hopeful than for the large coupled system of FBSDEs (5.4). In fact, the numerical approximation of mean field games has been one of the hot topics among the researchers in recent years. Moreover, if we adopt an appropriate linear-quadratic cost functions both for the major and minor agents, we may obtain an explicit form of the solution. Let us leave this problem as the potential future project.

\section{Securities with maturity $T$}

Let us briefly discuss the special case where the securities have exogenously specified payoff $c_{T}^{0} \in \mathbb{L}^{2}\left(\overline{\mathcal{F}}_{T}^{0} ; \mathbb{R}^{n}\right)$ at the date of maturity $T$. This is the situation arising in Futures, Bonds and many other financial derivatives. In this case, there is no reason to put a penalty on the terminal stock. It is then natural to consider

$$
\begin{aligned}
& g_{i}\left(x, c^{0}\right):=-\left\langle c^{0}, x\right\rangle, \quad 1 \leq i \leq N \\
& g_{0}^{(N)}\left(x, c^{0}\right)=\mathfrak{g}_{0}\left(x, c^{0}\right):=-\left\langle c^{0}, x\right\rangle,
\end{aligned}
$$

(i.e. $c_{i}^{g}(\cdot)=0$ ) for the terminal condition for the minor and the major agents, respectively. Since the terminal costs are linear in $x$, we now have $\gamma_{0}^{g}=\gamma^{g}=0$. Moreover, we remove the hard constraint $\beta_{T}=0$ from the major agent's admissible strategies. It does not play any role since there is no $\varpi$ dependence in the terminal cost functions for all the players. This means $\mathbb{A}^{0}=\mathbb{H}^{2}\left(\mathbb{F} ; \mathbb{R}^{n}\right)$ and $\mathbb{A}_{\mathrm{mfg}}^{0}=\mathbb{H}^{2}\left(\overline{\mathbb{F}}^{0} ; \mathbb{R}^{n}\right)$.

Although we loose strict convexity in the terminal functions, we can actually obtain the same conclusions also for this case. As we have already mentioned in [27], what we have to do is to apply Theorem 2.3 instead of Theorem 2.6 in [49]. Every theorem concerning the existence of the unique solution holds with the new terminal condition for the backward variables:

$$
\left\{\begin{aligned}
P_{T}^{0} & =-c_{T}^{0}, \\
Y_{T}^{i} & =-c_{T}^{0}, \\
P_{T}^{i} & =0 .
\end{aligned}\right.
$$

for the model with finite number of agents, and

$$
\left\{\begin{array}{l}
p_{T}^{0}=-c_{T}^{0}, \\
y_{T}^{1}=-c_{T}^{0}, \\
p_{T}^{1}=0,
\end{array}\right.
$$

for the model in the mean-field limit. Note that the verification theorem such as Theorems 4.3 and A.3 remain true since they do not require strict convexity in the terminal functions. In particular, (4.7) holds true with equality. 
One can easily check that the market-clearing price satisfies $\varpi_{T}=c_{T}^{0}$ in the both cases. The estimate in Theorem 5.3 is now given by, for every $1 \leq i \leq N$,

$$
\begin{gathered}
\mathbb{E}\left[\sup _{t \in[0, T]}\left(\left|\Delta x_{t}^{0}\right|^{2}+\left|\Delta x_{t}^{i}\right|^{2}+\left|\Delta r_{t}^{i}\right|^{2}+\left|\Delta p_{t}^{0}\right|^{2}+\left|\Delta y_{t}^{i}\right|^{2}+\left|\Delta p_{t}^{i}\right|^{2}\right)\right. \\
\left.\quad+\sum_{j=0}^{N} \int_{0}^{T}\left(\left|\Delta q_{t}^{0, j}\right|^{2}+\left|\Delta z_{t}^{i, j}\right|^{2}+\left|\Delta q_{t}^{i, j}\right|^{2}\right) \mathrm{d} t\right] \\
\leq C \mathbb{E}\left[\int_{0}^{T}\left(W_{2}\left(\bar{\mu}_{t}^{y, N}, \mu_{t}^{y}\right)^{2}+W_{2}\left(\bar{\mu}_{t}^{p, N}, \mu_{t}^{p}\right)^{2}\right) \mathrm{d} t\right] .
\end{gathered}
$$

One can prove it in the same way by using the new terminal condition; $\Delta p_{T}^{0}=\Delta y_{T}^{i}=\Delta p_{T}^{i}=0$.

\section{Appendix A. SufFicient MAXimum CONDITIONS FOR CONTROLLED-FBSDES}

Our optimization problem for the major agent requires the maximum principle for a system of controlledFBSDEs. The general issues of controlled-FBSDEs have been studied, in particular, by Yong [52, 53], where the second-order necessary conditions are given for non-convex control domain. In the current paper, we actually need the sufficient conditions (i.e. verification theorem) rather than the necessary conditions. On the other hand, we only need the convex control domain. Since we cannot find a useful summary in the existing literature, we provide the relevant theorem in this appendix. For the readers' convenience, we provide the theorem under the setup more general than what is actually needed for our purpose.

We let $(\Omega, \mathcal{F}, \mathbb{P}, \mathbb{F})$ be a complete filtered probability space satisfying the usual conditions. It supports a $d$-dimensional Brownian motion $W$ and $\mathcal{F}_{0}$ may be non-trivial. Let the control domain $A \subset \mathbb{R}^{k}$ be closed and convex and the space of admissible controls is denoted by $\mathbb{A}=\mathbb{H}^{2}(\mathbb{F} ; A)$. For a given $T>0$, we introduce the following measurable functions:

$$
\begin{aligned}
& b: \Omega \times[0, T] \times \mathbb{R}^{n} \times \mathbb{R}^{m} \times \mathbb{R}^{m \times d} \times A \rightarrow \mathbb{R}^{n}, \\
& \sigma: \Omega \times[0, T] \times \mathbb{R}^{n} \times \mathbb{R}^{m} \times \mathbb{R}^{m \times d} \times A \rightarrow \mathbb{R}^{n \times d}, \\
& f: \Omega \times[0, T] \times \mathbb{R}^{n} \times \mathbb{R}^{m} \times \mathbb{R}^{m \times d} \times A \rightarrow \mathbb{R}^{m}, \\
& \gamma: \Omega \times \mathbb{R}^{m} \rightarrow \mathbb{R}^{n}, \quad \Phi: \Omega \times \mathbb{R}^{n} \rightarrow \mathbb{R}^{m}, \quad \phi: \Omega \times \mathbb{R}^{m} \rightarrow \mathbb{R}^{m} .
\end{aligned}
$$

With these coefficient functions, we consider the following controlled system of FBSDEs:

$$
\left\{\begin{array}{l}
\mathrm{d} x_{t}=b\left(t, x_{t}, y_{t}, z_{t}, u_{t}\right) \mathrm{d} t+\sigma\left(t, x_{t}, y_{t}, z_{t}, u_{t}\right) \mathrm{d} W_{t}, \\
\mathrm{~d} y_{t}=f\left(t, x_{t}, y_{t}, z_{t}, u_{t}\right) \mathrm{d} t+z_{t} \mathrm{~d} W_{t}, \\
x_{0}=\gamma\left(y_{0}\right)+\xi \\
y_{T}=\Phi\left(x_{T}\right)+\phi\left(y_{0}\right)
\end{array}\right.
$$

where $\xi \in \mathbb{L}^{2}\left(\mathcal{F}_{0} ; \mathbb{R}^{n}\right)$ is given. See $[52,53]$ for various motivations to include the mixed initial-terminal conditions.

We study an optimization problem, $\inf _{\boldsymbol{u} \in \mathbb{A}} J(\boldsymbol{u})$, with

$$
J(\boldsymbol{u}):=\mathbb{E}\left[\int_{0}^{T} F\left(t, x_{t}, y_{t}, z_{t}, u_{t}\right) \mathrm{d} t+G\left(x_{T}\right)+g\left(y_{0}\right)\right]
$$


under the dynamic constraints (A.1). Here,

$$
\begin{aligned}
& F: \Omega \times[0, T] \times \mathbb{R}^{n} \times \mathbb{R}^{m} \times \mathbb{R}^{m \times d} \times A \rightarrow \mathbb{R}, \\
& G: \Omega \times \mathbb{R}^{n} \rightarrow \mathbb{R}, \quad g: \Omega \times \mathbb{R}^{m} \rightarrow \mathbb{R}
\end{aligned}
$$

are measurable functions representing the cost for the agent. The Hamiltonian $H: \Omega \times[0, T] \times \mathbb{R}^{n} \times \mathbb{R}^{m} \times$ $\mathbb{R}^{m \times d} \times \mathbb{R}^{n} \times \mathbb{R}^{n \times d} \times \mathbb{R}^{m} \times A \rightarrow \mathbb{R}$ is defined by

$$
\begin{aligned}
H(t, x, y, z, p, q, r, u):= & \langle p, b(t, x, y, z, u)\rangle+\langle q, \sigma(t, x, y, z, u)\rangle+\langle r, f(t, x, y, z, u)\rangle \\
& +F(t, x, y, z, u),
\end{aligned}
$$

where the brackets in the second term in the right-hand side denote a trace operation.

Assumption A.1. (i) For any $(x, y, z, u) \in \mathbb{R}^{n} \times \mathbb{R}^{m} \times \mathbb{R}^{m \times d} \times A,(b, \sigma, f, F)$ are $\mathbb{F}$-progressively measurable, $(\gamma, g)$ are $\mathcal{F}_{0}$-measurable and $\Phi, \phi, G$ are $\mathcal{F}_{T}$-measurable.

(ii) For any $\left(u_{t}\right)_{t \in[0, T]} \in \mathbb{A}$, there exists a unique strong solution $\left(x_{t}, y_{t}, z_{t}\right)_{t \in[0, T]} \in \mathbb{S}^{2}\left(\mathbb{F} ; \mathbb{R}^{n}\right) \times \mathbb{S}^{2}\left(\mathbb{F} ; \mathbb{R}^{m}\right) \times$ $\mathbb{H}^{2}\left(\mathbb{F} ; \mathbb{R}^{m \times d}\right)$ to the controlled FBSDE (A.1) .

(iii) $(b, \sigma, f, \gamma, \Phi, \phi)$ are one-time continuously differentiable in $(x, y, z, u)$ with bounded derivatives.

(iv) $(F, G, g)$ are one-time continuously differentiable in $(x, y, z, u)$ with uniformly Lipschitz continuous derivatives. Moreover, for any given $(x, y, z, u)$, these derivatives are square integrable.

(v) For any $\left(u_{t}\right)_{t \in[0, T]} \in \mathbb{A}, J(\boldsymbol{u})$ is finite.

(vi) $(G, g)$ are convex and $(\gamma, \Phi, \phi)$ are affine functions in $(x, y)$.

Remark A.2. For a scalar-valued function $f(x) \in \mathbb{R}$, we use the convention $f_{x}(x)=\left(\partial_{x^{i}} f(x)\right)_{i=1}^{n} \in \mathbb{R}^{n}$. For a vector-valued function $f(x) \in \mathbb{R}^{m}$, we use $f_{x}(x) \in \mathbb{R}^{m \times n}$ with $\left(f_{x}(x)\right)_{i, j}=\left(\partial_{x^{j}} f^{i}(x)\right)$.

The adjoint equations are given as follows:

$$
\left\{\begin{array}{l}
\mathrm{d} r_{t}=-H_{y}\left(t, x_{t}, y_{t}, z_{t}, p_{t}, q_{t}, r_{t}, u_{t}\right) \mathrm{d} t-H_{z}\left(t, x_{t}, y_{t}, z_{t}, p_{t}, q_{t}, r_{t}, u_{t}\right) \mathrm{d} W_{t} \\
\mathrm{~d} p_{t}=-H_{x}\left(t, x_{t}, y_{t}, z_{t}, p_{t}, q_{t}, r_{t}, u_{t}\right) \mathrm{d} t+q_{t} \mathrm{~d} W_{t} \\
r_{0}=\mathbb{E}\left[\phi_{y}\left(y_{0}\right)^{\top} r_{T} \mid \mathcal{F}_{0}\right]-\gamma_{y}\left(y_{0}\right)^{\top} p_{0}-g_{y}\left(y_{0}\right) \\
p_{T}=-\Phi_{x}\left(x_{T}\right)^{\top} r_{T}+G_{x}\left(x_{T}\right)
\end{array}\right.
$$

Theorem A.3. Let Assumption A.1 be in force. Suppose that $\left(\widehat{x}_{t}, \widehat{y}_{t}, \widehat{z}_{t}\right)_{t \in[0, T]} \in \mathbb{S}^{2} \times \mathbb{S}^{2} \times \mathbb{H}^{2}$ is a unique solution to the FBSDE (A.1) with some admissible control process $\left(\widehat{u}_{t}\right)_{t \in[0, T]} \in \mathbb{A}$. Assume that there exists a solution $\left(\widehat{p}_{t}, \widehat{q}_{t}, \widehat{r}_{t}\right)_{t \in[0, T]} \in \mathbb{S}^{2} \times \mathbb{H}^{2} \times \mathbb{S}^{2}$ to (A.2) with inputs $\left(\widehat{x}_{t}, \widehat{y}_{t}, \widehat{z}_{t}, \widehat{u}_{t}\right)_{t \in[0, T]}$, and that the map

$$
\mathbb{R}^{n} \times \mathbb{R}^{m} \times \mathbb{R}^{m \times d} \times A \ni(x, y, z, u) \mapsto H\left(t, x, y, z, \widehat{p}_{t}, \widehat{q}_{t}, \widehat{r}_{t}, u\right) \in \mathbb{R}
$$

is jointly convex in $(x, y, z, u)$ and strictly convex in $u, \mathrm{~d} t \otimes d \mathbb{P}$-a.e. Moreover, the equality

$$
H\left(t, \widehat{x}_{t}, \widehat{y}_{t}, \widehat{z}_{t}, \widehat{p}_{t}, \widehat{q}_{t}, \widehat{r}_{t}, \widehat{u}_{t}\right)=\inf _{u \in A} H\left(t, \widehat{x}_{t}, \widehat{y}_{t}, \widehat{z}_{t}, \widehat{p}_{t}, \widehat{q}_{t}, \widehat{r}_{t}, u\right)
$$

holds $\mathrm{d} t \otimes d \mathbb{P}$-a.e. Then, $\left(\widehat{u}_{t}\right)_{t \in[0, T]}$ is a unique optimal solution.

\footnotetext{
${ }^{2}$ For the existence of unique solutions to fully-coupled FBSDEs, see [49, 53]. In particular, the latter deals with the mixed initial-terminal conditions.
} 
Proof. Let us denote by $\left(x_{t}, y_{t}, z_{t}\right)_{t \in[0, T]}$ the unique solution to (A.1) with a given control process $\left(u_{t}\right)_{t \in[0, T]} \in \mathbb{A}$. For notational convenience, let us introduce

$$
\begin{aligned}
& \theta_{t}:=\left(x_{t}, y_{t}, z_{t}\right), \quad \widehat{\theta}_{t}:=\left(\widehat{x}_{t}, \widehat{y}_{t}, \widehat{z}_{t}\right), \quad \widehat{\varrho}_{t}:=\left(\widehat{p}_{t}, \widehat{q}_{t}, \widehat{r}_{t}\right), \\
& \widehat{\Theta}_{t}:=\left(\widehat{x}_{t}, \widehat{y}_{t}, \widehat{z}_{t}, \widehat{p}_{t}, \widehat{q}_{t}, \widehat{r}_{t}\right), \quad t \in[0, T] .
\end{aligned}
$$

Since $(\gamma, \Phi, \phi)$ are affine, we have

$$
\begin{aligned}
& \mathbb{E}\left[\left\langle G_{x}\left(\widehat{x}_{T}\right), x_{T}-\widehat{x}_{T}\right\rangle+\left\langle g_{y}\left(\widehat{y}_{0}\right), y_{0}-\widehat{y}_{0}\right\rangle\right] \\
& =\mathbb{E}\left[\left\langle\widehat{p}_{T}+\Phi_{x}\left(\widehat{x}_{T}\right)^{\top} \widehat{r}_{T}, x_{T}-\widehat{x}_{T}\right\rangle+\left\langle-\widehat{r}_{0}+\mathbb{E}\left[\phi_{y}\left(\widehat{y}_{0}\right)^{\top} \widehat{r}_{T} \mid \mathcal{F}_{0}\right]-\gamma_{y}\left(\widehat{y}_{0}\right)^{\top} \widehat{p}_{0}, y_{0}-\widehat{y}_{0}\right\rangle\right] \\
& =\mathbb{E}\left[\left\langle\widehat{p}_{T}, x_{T}-\widehat{x}_{T}\right\rangle-\left\langle\widehat{p}_{0}, x_{0}-\widehat{x}_{0}\right\rangle+\left\langle\widehat{r}_{T}, y_{T}-\widehat{y}_{T}\right\rangle-\left\langle\widehat{r}_{0}, y_{0}-\widehat{y}_{0}\right\rangle\right],
\end{aligned}
$$

where we have used the relation, for example, $\Phi_{x}\left(\widehat{x}_{T}\right)\left(x_{T}-\widehat{x}_{T}\right)=\Phi\left(x_{T}\right)-\Phi\left(\widehat{x}_{T}\right)$.

Now, Itô-formula gives

$$
\begin{aligned}
\mathbb{E} & {\left[\left\langle\widehat{p}_{T}, x_{T}-\widehat{x}_{T}\right\rangle-\left\langle\widehat{p}_{0}, x_{0}-\widehat{x}_{0}\right\rangle+\left\langle\widehat{r}_{T}, y_{T}-\widehat{y}_{T}\right\rangle-\left\langle\widehat{r}_{0}, y_{0}-\widehat{y}_{0}\right\rangle\right] } \\
= & \mathbb{E} \int_{0}^{T}\left[\left\langle\widehat{p}_{t}, b\left(t, \theta_{t}, u_{t}\right)-b\left(t, \widehat{\theta}_{t}, \widehat{u}_{t}\right)\right\rangle-\left\langle H_{x}\left(t, \widehat{\Theta}_{t}, \widehat{u}_{t}\right), x_{t}-\widehat{x}_{t}\right\rangle+\left\langle\widehat{q}_{t}, \sigma\left(t, \theta_{t}, u_{t}\right)-\sigma\left(t, \widehat{\theta}_{t}, \widehat{u}_{t}\right)\right\rangle\right. \\
& \left.+\left\langle\widehat{r}_{t}, f\left(t, \theta_{t} u_{t}\right)-f\left(t, \widehat{\theta}_{t}, \widehat{u}_{t}\right)\right\rangle-\left\langle H_{y}\left(t, \widehat{\Theta}_{t}, \widehat{u}_{t}\right), y_{t}-\widehat{y}_{t}\right\rangle-\left\langle H_{z}\left(t, \widehat{\Theta}_{t}, \widehat{u}_{t}\right), z_{t}-\widehat{z}_{z}\right\rangle\right] \mathrm{d} t .
\end{aligned}
$$

It is easy to check that the stochastic integration part becomes a true martingale. Using the convexity of $G$ and $g$, we have

$$
\begin{aligned}
& J(\boldsymbol{u})-J(\widehat{\boldsymbol{u}}) \\
& \geq \mathbb{E}\left[\left\langle G_{x}\left(\widehat{x}_{T}\right), x_{T}-\widehat{x}_{T}\right\rangle+\left\langle g_{y}\left(\widehat{y}_{0}\right), y_{0}-\widehat{y}_{0}\right\rangle+\int_{0}^{T}\left[F\left(t, \theta_{t}, u_{t}\right)-F\left(t, \widehat{\theta}_{t}, \widehat{u}_{t}\right)\right] \mathrm{d} t\right] \\
&=\mathbb{E} \int_{0}^{T}\left[H\left(t, \theta_{t}, \widehat{\varrho}_{t}, u_{t}\right)-H\left(t, \widehat{\Theta}_{t}, \widehat{u}_{t}\right)-\left\langle H_{x}\left(t, \widehat{\Theta}_{t}, \widehat{u}_{t}\right), x_{t}-\widehat{x}_{t}\right\rangle\right. \\
&\left.\quad-\left\langle H_{y}\left(t, \widehat{\Theta}_{t}, \widehat{u}_{t}\right), y_{t}-\widehat{y}_{t}\right\rangle-\left\langle H_{z}\left(t, \widehat{\Theta}_{t}, \widehat{u}_{t}\right), z_{t}-\widehat{z}_{t}\right\rangle\right] \mathrm{d} t \\
& \geq \mathbb{E} \int_{0}^{T}\left[H\left(t, \theta_{t}, \widehat{\varrho}_{t}, u_{t}\right)-H\left(t, \widehat{\Theta}_{t}, \widehat{u}_{t}\right)-\left\langle H_{x}\left(t, \widehat{\Theta}_{t}, \widehat{u}_{t}\right), x_{t}-\widehat{x}_{t}\right\rangle\right. \\
&\left.\quad-\left\langle H_{y}\left(t, \widehat{\Theta}_{t}, \widehat{u}_{t}\right), y_{t}-\widehat{y}_{t}\right\rangle-\left\langle H_{z}\left(t, \widehat{\Theta}_{t}, \widehat{u}_{t}\right), z_{t}-\widehat{z}_{t}\right\rangle-\left\langle H_{u}\left(t, \widehat{\Theta}_{t}, \widehat{u}_{t}\right), u_{t}-\widehat{u}_{t}\right\rangle\right] \mathrm{d} t \\
& \geq 0, \quad
\end{aligned}
$$

where the equality hods if and only if $(u=\widehat{u})$ due to the strict convexity.

\section{REFERENCES}

[1] C. Alasseur, I. Ben Taher and A. Matoussi, An extended mean field games for storage in smart grids. J. Optim. Theory Appl. 184 (2020) 644-670.

[2] Y. Achdou, M. Bardi and M. Cirant, Mean field games models of segregation. Math. Models Methods Appl. Sci. 27 (2017) $75-113$.

[3] A. Bensoussan, J. Frehse and P. Yam, Mean field games and mean field type control theory. SpringerBriefs in Mathematics, NY (2013). 
[4] A. Bensoussan, M.H.M. Chau and S.C.P. Yam, Mean field games with a dominating player. Appl. Math. Optim. 74 (2016) 91-128.

[5] A. Bensoussan, T. Huang and M. Lauriere, Mean field control and mean field game models with several populations. Minimax Theory Appl. 03 (2018) 173-209.

[6] P. Cardaliaguet, Notes on Mean Field Games (2013). Available at https://www.ceremade.dauphine.fr/ $\sim_{\text {cardaliaguet }}$

[7] P. Cardaliaguet, M. Cirant and A. Porretta, Remarks on Nash equilibria in mean field game models with a major player. Proc. Amer. Math. Soc. 148 (2020) 4241-4255.

[8] R. Carmona and F. Delarue, Probabilistic analysis of mean-field games. SIAM J. Control. Optim. 51 (2013) $2705-2734$.

[9] R. Carmona and F. Delarue, Forward-backward stochastic differential equations and controlled McKean-Vlasov dynamics. Ann. Probab. 43 (2015) 2647-2700.

[10] R. Carmona and F. Delarue, Probabilistic Theory of Mean Field Games with Applications I. Springer International Publishing, Switzerland (2018).

[11] R. Carmona and F. Delarue, Probabilistic Theory of Mean Field Games with Applications II. Springer International Publishing, Switzerland (2018)

[12] R. Carmona, F. Delarue and D. Lacker, Mean field games with common noise. Ann. Probab. 44 (2016) $3740-3803$.

[13] R. Carmona and X. Zhu, A probabilistic approach to mean field games with major and minor players. Ann. Appl. Probab. 26 (2016) 1535-1580.

[14] P. Casgrain and S. Jaimungal, Mean-field games with differing beliefs for algorithmic trading. Math. Finance 30 (2020) 995-1034.

[15] M. Cirant, Multi-population mean field games system with Neumann boundary conditions. J. Math. Pures Appl. 103 (2015) $1294-1315$

[16] B. Djehiche, J. Barreiro-Gomez and H. Tembine, Electricity price dynamics in the smart grid: a mean-field-type game perspective, 23rd International Symposium on Mathematical Theory of Networks and Systems Hong Kong University of Science and Technology, Hong Kong, July 16-20, 2018 (2018).

[17] M.F. Djete, Mean field games of controls: on the convergence of Nash equilibria. Preprint arXiv:2006.12993 (2020).

[18] M.F. Djete, Extended mean field control problems: a propagation of chaos result. Preprint arXiv:2006.12996 (2020).

[19] D. Evangelista and Y. Thamsten, On finite population games of optimal trading. Preprint arXiv:2004.00790 (2020).

[20] E. Feleqi, The derivation of ergodic mean field game equations for several population of players. Dyn. Games Appl. 3 (2013) $523-536$.

[21] O. Féron, P. Tankov and L. Tinsi, Price formation and optimal trading in intraday electricity markets. Preprint arXiv:2009.0478 (2020).

[22] O. Féron, P. Tankov and L. Tinsi, Price formation and optimal trading in intraday electricity markets with a Major Player. Risks 8 (2020) 133.

[23] D. Firoozi, S. Jaimungal and P. Caines, Convex analysis for LQG systems with applications to major-minor LQG mean-field game systems. Syst. Control Lett. 142 (2020) 104734.

[24] G. Fu, P. Graewe, U. Horst and A. Popier, A mean field game of optimal portfolio liquidation. Math. Oper. Res. (2021) 1-32.

[25] G. Fu, U. Horst, Mean-field leader-follower games with terminal state constraint. SIAM J. Control. Optim. 58 (2018) 20782113.

[26] M. Fujii, Probabilistic approach to mean field games and mean field type control problems with multiple populations. To appear in Minimiax Theory Appl. (2019).

[27] M. Fujii and A. Takahashi, A mean field game approach to equilibrium pricing with market clearing condition. To appear in SIAM J. Control Optim. (2020).

[28] M. Fujii and A. Takahashi, Strong convergence to the mean-field limit of a finite agent equilibrium. The paper was originally titled as "A finite agent equilibrium in an incomplete market and its strong convergence to the mean-field limit". To appear SIAM J. Financial Math. (2020).

[29] D.A. Gomes, L. Nurbekyan and E.A. Pimentel, Economic models and mean-field games theory. Publicaoes Matematicas, IMPA, Rio, Brazil (2015).

[30] D.A. Gomes, E.A. Pimental and V. Voskanyan, Regularity Theory for Mean-field game systems. SpringerBriefs in Mathematics (2016).

[31] D.A. Gomes and J. Saude, A mean-field game approach to price formation. Dyn. Games Appl. (2020). https://doi.org/10. 1007/s13235-020-00348-x.

[32] O. Gueant, J. Lasry and P. Lions, Mean field games and oil production. Economica (2010).

[33] M. Huang, Large-population LQG games involving a major player: the Nash certainty equivalence principle. SIAM. J. Control. Optim. 48 (2010) 3318-3353.

[34] M. Huang, R. Malhame and P.E. Caines, Large population stochastic dynamic games: closed-loop McKean-Vlasov systems and the Nash certainty equivalence principle. Commun. Inf. Syst. 6 (2006) 221-252.

[35] M. Huang, R. Malhame and P.E. Caines, Nash certainty equivalence in large population stochastic dynamic games: connections with the physics of interacting particle systems. Proceedings of the 45th IEEE Conference on Decision and Control (2006) 4921-4926.

[36] M. Huang, R. Malhame and P.E. Caines, An invariance principle in large population stochastic dynamic games. J. Syst. Sci. Complexity 20 (2007) 162-172. 
[37] M. Huang, R. Malhame and P.E. Caines, Large-population cost-coupled LQG problems with nonuniform agents: individualmass behavior and decentralized $\epsilon$-Nash equilibria. IEEE Trans. Autom. Control 52 (2007) 1560-1571.

[38] V.N. Kolokoltsov and O.A. Malafeyev, Many agent games in socio-economic systems: corruption, inspection, coalition building, network growth, security. Springer Series in Operations Research and Financial Engineering (2019).

[39] D. Lacker, Mean field games via controlled martingales problems: Existence of Markovian equilibria. Stochastic Process. Appl. 125 (2015) 2856-2894.

[40] D. Lacker, A general characterization of the mean field limit for stochastic differential games. Probab. Theory Relat. Fields 165 (2016) 581-648.

[41] J. M. Lasry and P.L. Lions, Jeux a champ moyen I. Le cas stationnaire. C. R. Sci. Math. Acad. Paris 343 (2006) $619-625$.

[42] J.M. Lasry and P.L. Lions, Jeux a champ moyen II. Horizon fini et controle optimal. C. R. Sci. Math. Acad. Paris 343 (2006) 679-684.

[43] J.M. Lasry and P.L. Lions, Mean field games. Jpn. J. Math. 2 (2007) 229-260.

[44] J.M. Lasry and P.L. Lions, Mean-field games with a major player. Comptes Rendus Math. 356 (2018) 886-890.

[45] C.A. Lehalle and C. Mouzouni, A mean field game of portfolio trading and its consequences on perceived correlations. Available at https://arxiv.org/pdf/1902.09606.pdf (2019).

[46] M. Laurière and L. Tangi, Convergence of large population games to mean field games with interaction through the controls. Preprint arXiv:2004.0835 (2020).

[47] M. Nourian and P. Caines, $\epsilon$-Nash mean field game theory for nonlinear stochastic dynamical systems with major and minor agents. SIAM. J. Control. Optim. 51 (2020) 3302-3331.

[48] A. Shrivats, D. Firoozi and S. Jaimungal, A mean-field game approach to equilibrium pricing, optimal generation, and trading in solar renewable energy certificate markets. Preprint arXiv:2003.04938 (2020).

[49] S. Peng and Z. Wu, Fully coupled forward-backward stochastic differential equations and applications to optimal control. SIAM J. Control Optim. 37 (1999) 825-843.

[50] B.H. Xing and G. Žitković, A class of globally solvable Markovian quadratic bsde systems and applications. Ann. Prob. 46 (2018) 491-550.

[51] J. Yong, Finding adapted solutions of forward-backward stochastic differential equations: method of continuation. Probab. Theory Related Fields 107 (1997) 537-572.

[52] J. Yong, Optimality variational principle for controlled forward-backward stochastic differential equations with mixed initialterminal conditions. SIAM J. Control. Optim. 48 (2010) 4119-4156.

[53] J. Yong, Forward-backward stochastic differential equations with mixed initial-terminal conditions. Trans. Am. Math. Soc. 362 (2010) 1047-1096.

[54] X. Yu, Y. Zhang and Z. Zhou, Teamwise mean field competitions. arXiv:2006.14472 (2020).

[55] K. Weston and G. Žitković, An incomplete equilibrium with a stochastic annuity. Finance Stoch. 24 (2020) 359-382.

[56] J. Zhang, Backward Stochastic Differential Equations. Springer, NY (2017).

\section{Subscribe to Open (S2O)}

\section{A fair and sustainable open access model}

This journal is currently published in open access under a Subscribe-to-Open model (S2O). S2O is a transformative model that aims to move subscription journals to open access. Open access is the free, immediate, online availability of research articles combined with the rights to use these articles fully in the digital environment. We are thankful to our subscribers and sponsors for making it possible to publish this journal in open access, free of charge for authors.

\section{Please help to maintain this journal in open access!}

Check that your library subscribes to the journal, or make a personal donation to the S2O programme, by contacting subscribers@edpsciences.org

More information, including a list of sponsors and a financial transparency report, available at: https://www.edpsciences.org/en/maths-s2o-programme 